\title{
Basic Science of PET Imaging for Inflammatory Diseases
}

\author{
Kazuo Kubota, Mikako Ogawa, Bin Ji, Tadashi Watabe, \\ Ming-Rong Zhang, Hiromi Suzuki, Makoto Sawada, \\ Kodai Nishi, and Takashi Kudo
}

\subsection{Mechanisms of FDG Accumulation in Inflammation}

\section{Kazuo Kubota}

\begin{abstract}
FDG-PET/CT has recently emerged as a useful tool for the evaluation of inflammatory diseases too, in addition to that of malignant diseases. The imaging is based on active glucose utilization by inflammatory tissue. Autoradiography studies have demonstrated high FDG uptake in macrophages, granulocytes, fibroblasts, and granulation tissue. Especially, activated macrophages are responsible for the elevated FDG uptake in some types of inflammation. According to one study, after activation by lipopolysaccharide of cultured macrophages, the $\left[{ }^{14} \mathrm{C}\right] 2 \mathrm{DG}$ uptake by the cells doubled, reaching the level seen in glioblastoma cells. In activated macrophages, increase in the expression of total GLUT1 and redistributions from the intracellular compartments toward the cell surface have been reported. In one rheumatoid arthritis model, following stimulation by hypoxia or $T N F-\alpha$, the highest elevation of the $\left[{ }^{3} \mathrm{H}\right] \mathrm{FDG}$ uptake was observed in the fibroblasts, followed by
\end{abstract}

\section{K. Kubota $(\bowtie)$}

Department of Radiology, Southern TOHOKU General Hospital,

Koriyama, Fukushima, Japan

e-mail: kkubota@cpost.plala.or.jp

M. Ogawa

Laboratory of Bioanalysis and Molecular Imaging, Graduate

School of Pharmaceutical Sciences, Hokkaido University,

Sapporo, Hokkaido, Japan

e-mail:mogawa@pharm.hokudai.ac.jp

B. Ji

Department of Functional Brain Imaging Research (DOFI),

National Institute of Radiological Sciences, National Institutes for

Quantum and Radiological Science and Technology, Chiba, Japan

e-mail: Ji.bin@qst.go.jp

T. Watabe

Department of Nuclear Medicine and Tracer Kinetics, Osaka

University Graduate School of Medicine, Osaka, Japan

e-mail: watabe@tracer.med.osaka-u.ac.jp that in macrophages and neutrophils. As the fundamental mechanism of elevated glucose uptake in both cancer cells and inflammatory cells, activation of glucose metabolism as an adaptive response to a hypoxic environment has been reported, with transcription factor $\mathrm{HIF}-1 \alpha$ playing a key role. Inflammatory cells and cancer cells seem to share the same molecular mechanism of elevated glucose metabolism, lending support to the notion of usefulness of FDGPET/CT for the evaluation of inflammatory diseases, besides cancer.

Keywords: Macrophage, Neutrophil, Fibroblast, Granulation tissue, GLUT, HIF-1 $\alpha$, FDG

\subsubsection{Introduction}

Fluorine-18-labeled 2-deoxy-2-fluoro-glucose (FDG) is used as a radiopharmaceutical in PET for evaluating glucose metabolism, and accumulates in malignant tissues because of the enhanced glucose utilization by neoplastic cells. Because of the increased metabolic demand for glucose,

M.-R. Zhang

Department of Radiopharmaceuticals Development, National Institute of Radiological Sciences, National Institutes for Quantum and Radiological Science and Technology, Chiba, Japan e-mail: zhang.ming-rong@qst.go.jp

H. Suzuki · M. Sawada

Division of Stress Adaptation and Protection, Department of Brain Function, Research Institute of Environmental Medicine,

Nagoya University, Nagoya, Aichi, Japan e-mail: hiromi_s@ riem.nagoya-u.ac.jp; msawada@riem.nagoya-u.ac.jp

K. Nishi · T. Kudo

Department of Radioisotope Medicine, Atomic Bomb Disease Institute, Nagasaki University, Nagasaki, Japan e-mail: koudai@nagasaki-u.ac.jp; tkudo123@nagasaki-u.ac.jp 
elevated activity of hexokinase and elevated expression of glucose transporter have been shown in tumor tissues [1]. Various applications of FDG-PET have been extensively studied in the field of clinical oncology, and the imaging modality, now used worldwide, is recognized as a powerful diagnostic modality for cancer [2, 3]. In 1989, elevated FDG uptake was reported in two patients with abdominal abscesses [4]. This was followed by reports of FDG uptake in brain abscess [5], tuberculosis, aspergillosis, sarcoidosis, and so on. In addition, early postoperative scarring and early inflammatory reactions after radiotherapy were also reported to show increased FDG uptake. Thus, elevation in glucose metabolism is not only specific for cancer but also seen in inflammation. Because FDG uptake is seen in benign inflammatory diseases as well, the accuracy of FDG-PET for the diagnosis of cancer is not $100 \%$ [6].

\subsubsection{FDG Uptake by Inflammatory Tissues and Cells}

Although the metabolic fate of FDG is well known, its cellular distribution within tumors or sites of inflammation has not yet been described. To clarify the mechanisms of FDG
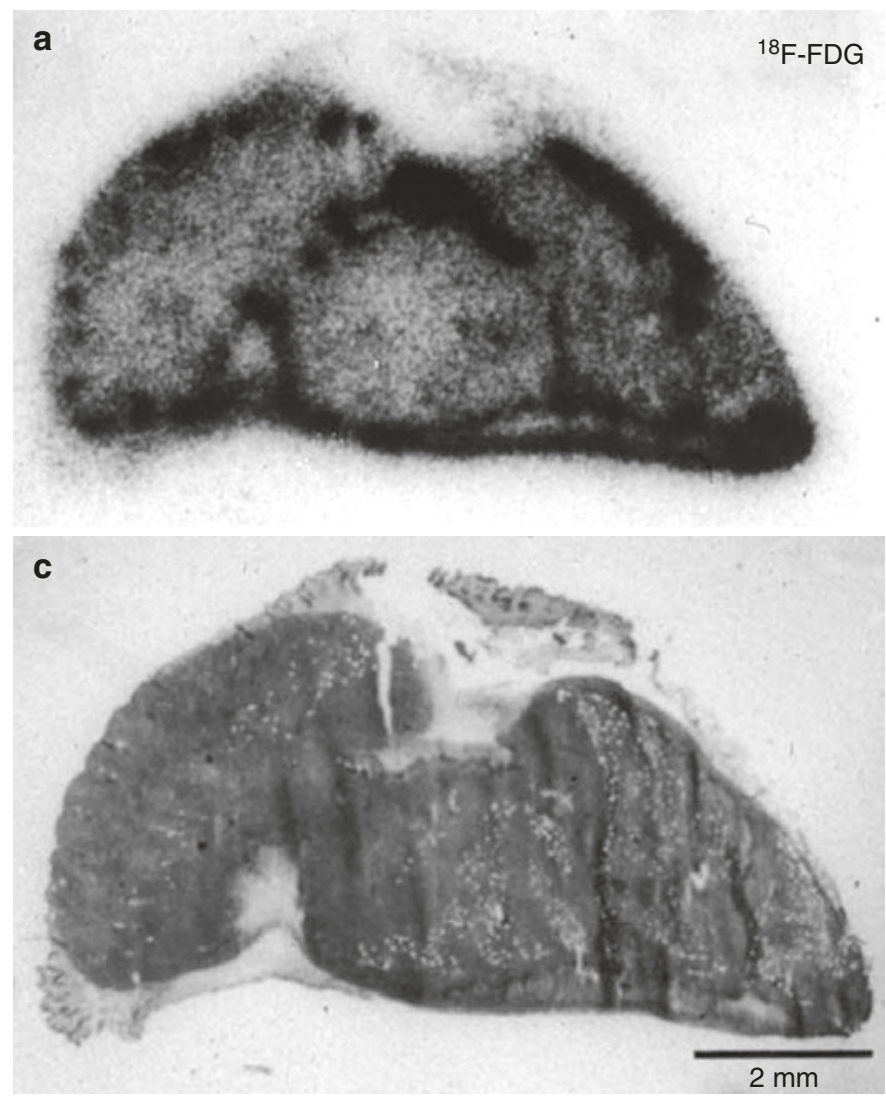

Fig. 1.1 A combination of double-tracer macro-autoradiograms and microscopy. Images of 18F-FDG distribution (a) and 3HThd (b), a photomicrograph of the tissue specimen (c) and an illustration of the micrograph (d). $T$ tumor calls, $G$ granulation tissue, $N$ necrosis, $H$ host normal uptake by inflammatory and tumor tissues, we performed autoradiographic studies. To demonstrate the cellular localization of FDG and $\left[{ }^{3} \mathrm{H}\right] 2 \mathrm{DG}$ uptake by tumors in vivo, $\mathrm{C} 3 \mathrm{H} /$ $\mathrm{He}$ mice with subcutaneously transplanted FM3A tumors were studied $1 \mathrm{~h}$ after intravenous injection of FDG or $\left[{ }^{3} \mathrm{H}\right] 2 \mathrm{DG}$. Newly formed granulation tissue around tumors and macrophages, which had massively infiltrated the marginal areas surrounding the necrotic areas of the tumor, showed a higher FDG uptake than the viable tumor cells. A maximum of $24 \%$ of the glucose utilization was derived from the non-neoplastic tissues in these tumors (Fig. 1.1). The strong accumulation of FDG in these tumors was thought to represent both the high metabolic activity of the viable tumor cells and that of the tumor-associated inflammatory cells, especially activated macrophages. These results indicate that not only glucose uptake by the tumor cells but also that by non-neoplastic cellular elements which appear in association with the growth or necrosis of tumor cells should be considered for a precise analysis of FDG uptake in tumors, especially after radiotherapy (Fig. 1.2) [7, 8].

FDG uptake by inflammatory tissues was investigated by Yamada et al. [9]. A rat model of chemically induced inflammation using turpentine oil was used. A time-course study of the FDG tissue distribution showed that the uptake of FDG
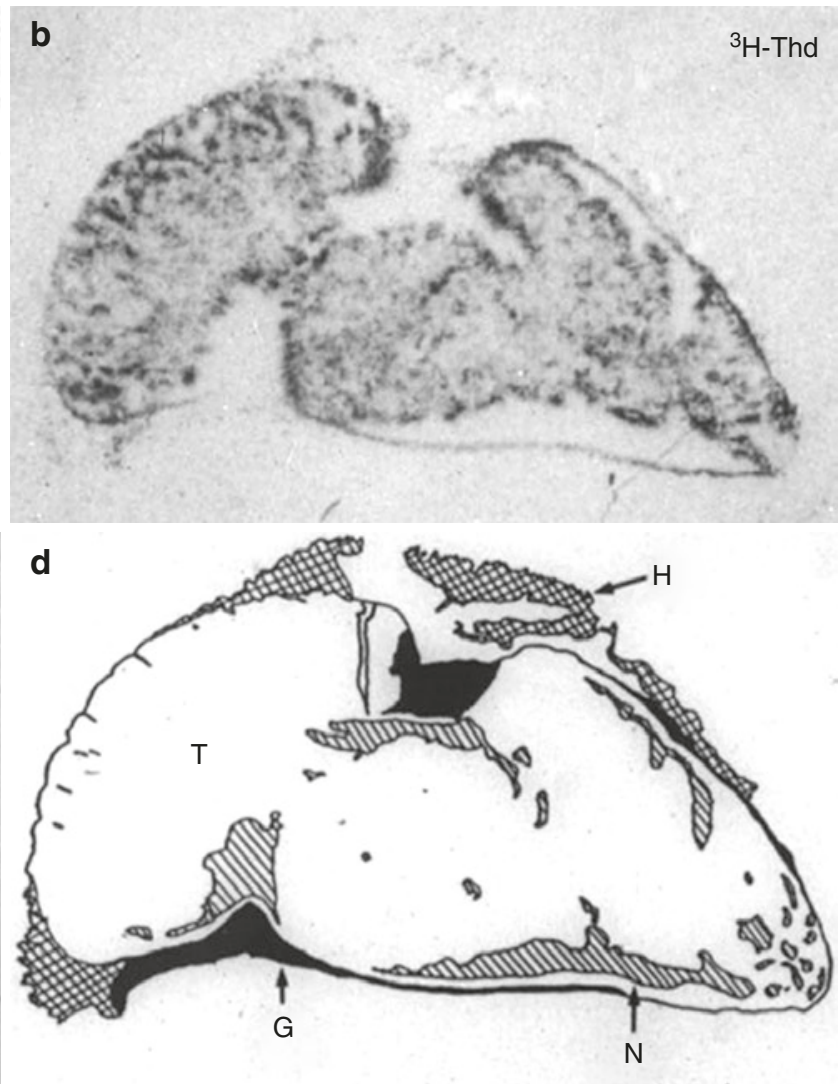

tissue. Scale bar: $2 \mathrm{~mm}$. Newly formed granulation tissue around the tumor and macrophages infiltrating the periphery of the necrotic areas of the tumor showed higher uptakes of FDG than the viable tumor cells. (From Ref. [7]) 
in inflammatory tissue increased gradually until $60 \mathrm{~min}$, followed by a steady decrease thereafter. A longitudinal study showed that the uptake increased progressively after the start of inflammation, peaking at 4 days after the inoculation, and then gradually decreased (Fig. 1.3). These findings suggested that FDG uptake may reach a maximum during the subacute phase of inflammation, and then slightly decreases during the chronic phase of inflammation. An autoradiography study showed a high FDG uptake in the abscess wall, consisting of an inflammatory cell layer and granulation tissue. At the cellular level, the highest radioactivity was found in the marginal zone that contained young fibroblasts, endothelial cells of vessels, and phagocytes consisting of neutrophils and macrophages, followed by that in the neutrophil layer and the granulation tissue layer (Fig. 1.4). FDG uptake by

\section{Neoplastic tissue}

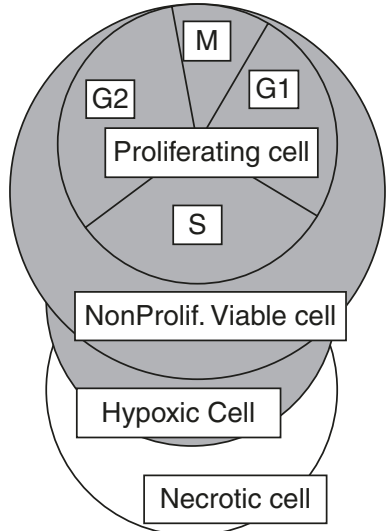

FDG uptake

\section{Interstitial tissue (Inflammation)}

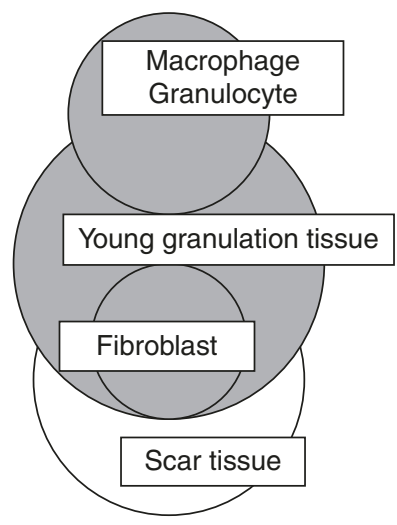

No FDG uptake
Fig. 1.2 A model of FDG accumulation in various cellular elements in a tumor. (Modified from Ref. [8])

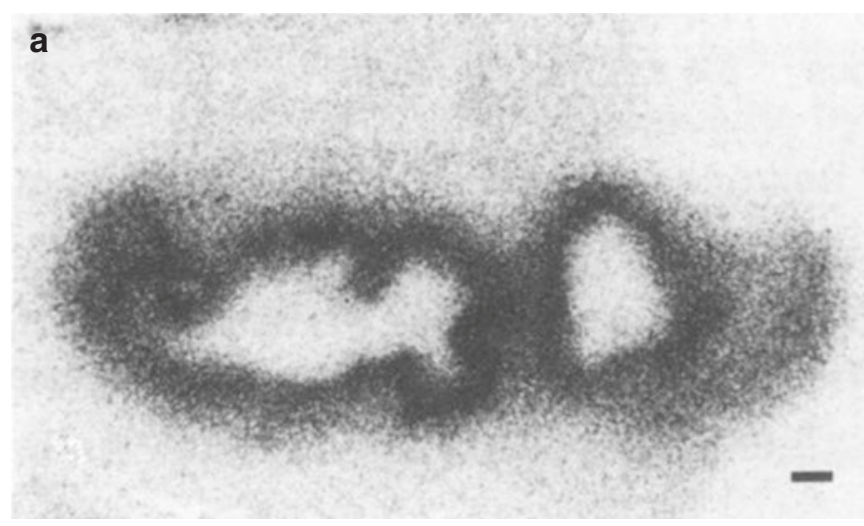

Fig. 1.4 Macro-autoradiogram (a) and the corresponding section (b) of inflammatory tissue 4 days after inoculation of turpentine oil. The center (C), surrounded by inflammatory cells (IC), thick granulation inflammatory tissue seems to represent the activity of immune cells and fibroblasts mobilized to the lesion.

Mochizuki et al. [10] compared the FDG uptake by experimental hepatoma and by tissue with experimental infection with Staphylococcus aureus. The expressions of GLUT1 and GLUT3 were also studied in both the tumor and infected tissue by immunostaining. Uptake by the tumor tissue was significantly higher than that by the infected tissue. Both the tumor and infected tissue showed strong expression of GLUT1 and GLUT 3, although the expression level of GLUT1 was significantly higher in the tumor than in the infected tissue. GLUT1 may be responsible for FDG uptake in both tumor tissue and infected tissue.

Zhao et al. [11] compared the two models of inflammation: BCG-induced granuloma simulating sarcoidosis and

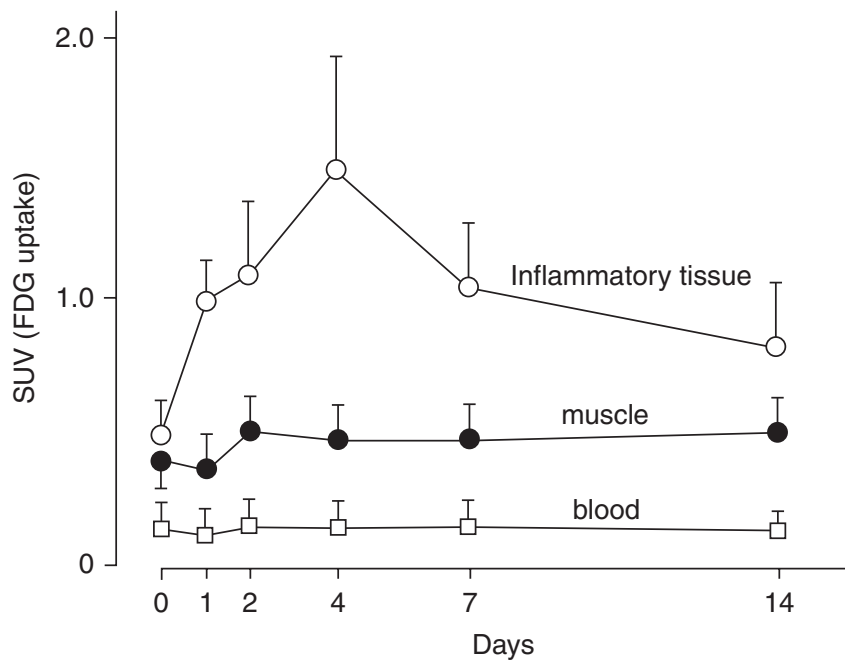

Fig. 1.3 FDG uptake changes along with the age of inflammation. FDG uptake by inflammatory tissue, muscle, and blood are plotted against the days after inoculation of turpentine oil. The highest uptake by inflammatory tissue was observed on day 4 , which represented the subacute phase histologically ( $n=5$, each point). (From Ref. [9])

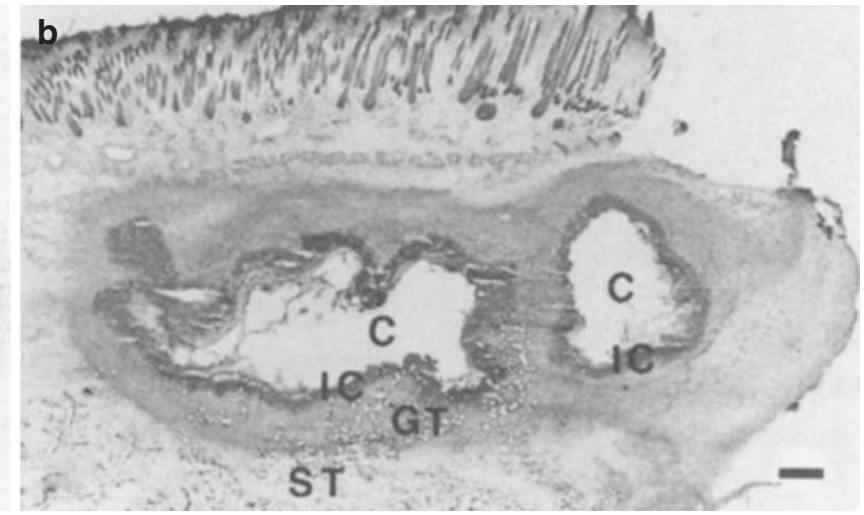

tissue (GT), and edematous subcutaneous tissue (ST). Scale bar 0.4 mm. (From Ref. [9]) 
turpentine oil-induced inflammation. FDG uptake by the granuloma was significantly higher than that by the tissue with chemical-induced inflammation. Their results explained the very high FDG uptake in sarcoidosis, equivalent to the uptake in cancer. However, the main objective of their study was not to compare the models of inflammation, but to compare the uptakes of ${ }^{14} \mathrm{C}$-methionine, ${ }^{18} \mathrm{~F}$-fluorothymidine, and FDG. ${ }^{14} \mathrm{C}$-methionine showed the best results for discrimination between tumor and inflammation.

\subsubsection{Activated Macrophages and GLUT}

Deichen et al. [12] studied the uptake of FDG in isolated human monocyte-macrophages (HMMs) in vitro. FDG uptake by the HMMs increased significantly with the duration of culture, the percent ID/100 $\mu \mathrm{g}$ being $7.5 \% \pm 0.9 \%(\%$ ID/100 $\mu \mathrm{g}$ ) on day 14. Stimulation by lipopolysaccharide further enhanced the FDG uptake in the HMMs by a factor of 2. Radio-thin layer chromatography of intracellular metabolites revealed that the FDG was trapped by the HMMs mainly as FDG-6-phosphate and FDG-1,6-diphosphate. FDG uptake by the HMMs was almost equal to that by human glioblastoma and pancreatic carcinoma cells.

Malide et al. [13] reported the changes in the subcellular localization of the glucose transporter proteins GLUT-1, GLUT-3, and GLUT-5 as the human monocytes differentiated into macrophages in culture, and the effects of the activating agents $N$-formyl-methionyl-leucylphenylalanine (fMLP) and phorbol myristate acetate (PMA). Western blot analysis demonstrated progressively increasing GLUT-1 expression, rapidly decreasing GLUT-3 expression, and a delayed increase of the GLUT-5 expression during the differentiation process. Confocal microscopy revealed that each isoform exhibited a unique subcellular distribution and cell-activation response. GLUT-1 was localized primarily at the cell surface, but was also detected in the perinuclear region, in a pattern characteristic of recycling endosomes. Activation with fMLP induced similar GLUT-1 and GLUT-5 redistributions from the intracellular compartments toward the cell surface. Addition of PMA elicited a similar translocation of GLUT-1, but GLUT-5 was redistributed from the plasma membrane to a distinct intracellular compartment that appeared connected to the cell surface. These results suggest specific subcellular targeting of each transporter isoform and differential regulation of their trafficking pathways in cultured macrophages.

\subsubsection{Activated Neutrophils}

Not only macrophages but also neutrophil granulocytes play a key role in the pathogenesis of various types of inflammation. Jones et al. [14] studied [ $\left.{ }^{3} \mathrm{H}\right] \mathrm{DG}$ uptake in neutrophils isolated from human peripheral blood. They found elevated uptake of $\left[{ }^{3} \mathrm{H}\right] \mathrm{DG}$ by neutrophils, both after priming with tumor necrotic factor-alpha (TNF- $\alpha)$ and after activation with fMLP. It was not correlated with the respiratory burst or secretory activity, but may reflect the polarization and migrational status of these cells. Their study suggested that as far as neutrophils are concerned, priming is the cellular event predominantly responsible for the FDG uptake. Ishimori et al. [15] studied immunocompetent BALB/c mice and nude mice administered an intravenous injection of $10 \mathrm{mg} / \mathrm{kg}$ of concanavalin A (Con A). After the injection, the Con A-activated lymphocytes actively took up FDG both in vitro and in vivo, and FDG specifically accumulated in the tissues showing Con A-mediated acute inflammation in the immunocompetent mice.

\subsubsection{Activated Fibroblasts}

Rheumatoid arthritis (RA) is an autoimmune disorder of unknown etiology and is characterized by systematic, symmetric, and erosive synovitis. RA synovitis is characterized by massive leukocyte infiltration, proliferative synovial membranes, and neovascularization, which give rise to a synovial proliferative fibrovascular tissue known as a pannus. Formation of pannus is directly responsible for the cartilage and bone destruction [16]. Matsui et al. [17] reported the mechanism of FDG accumulation in RA in vivo using a murine collagen-induced arthritis model, as well as $\left[{ }^{3} \mathrm{H}\right]$ FDG uptake in vitro using various cell types. They showed that FDG accumulation increased with the progression of joint swelling. FDG uptake began in the area of inflammatory cell infiltration and synovial cell hyperplasia, and the areas showing strong FDG uptake often coincided with the areas where mixed cellular patterns of macrophages and fibroblasts as well as bone destruction by mature osteoclasts were visible. These findings indicated that FDG accumulation reflected the characteristic changes of pathological progression, such as pannus formation and bone destruction. Based on the findings of in vitro experiments, Matsui et al. suggested that the cell types responsible for FDG uptake were mostly proliferating fibroblasts, with lesser contributions from activated macrophages. FDG uptake by fibroblasts was enhanced in the presence of cytokine stimulation and hypoxia within a joint. Hypoxia is a known feature of the microenvironment in inflamed joints [18]. Recently, Garcia-Carbonell et al. directly compared fibroblast-like synoviocytes (FLS) from RA patients and osteoarthritis patients [19]. In vitro experiments have shown that the FLS from RA patients were dependent on glycolysis rather than on oxidative phosphorylation, and this difference was more pronounced than that for the FLS from osteoarthritis patients. The expression of GLUT-1 messenger RNA was 
correlated with the functions of the FLS from the RA patients. These studies showed the importance of fibroblasts in the inflammation in RA.

\subsubsection{Contribution of HIF-1 $\alpha$}

Recently, the processes, at the molecular level, occurring in association with hypoxia and glucose metabolism within tumor and inflammatory tissues have been described. Cramer et al. reported that activation of hypoxia-inducible factor one-alpha $(\mathrm{HIF}-1 \alpha)$ is essential for myeloid cell infiltration and activation in vivo [20]. They showed that HIF- $1 \alpha$ is essential for regulation of the glycolytic capacity of myeloid cells; when HIF-1 $\alpha$ is knocked out, the cellular ATP pool decreases drastically. This metabolic defect results in a profound impairment of myeloid cell aggregation, motility, invasiveness, and bacterial killing at sites of inflammation where the tissue environment is hypoxic. Thus, HIF- $1 \alpha$ has a direct regulatory effect on both the survival and functioning of cells in inflammatory microenvironments. Furthermore, the enhanced glycolysis in activated macrophages results in elevation of the FDG uptake. Regarding the molecular mechanisms responsible for the regulation of glycolysis, HIF-1 $\alpha$ affects both glucose transporter and hexokinase expressions in tumors and inflamed tissues.

The significance of hypoxia in tumor pathophysiology has also been described by Denko [21]. Usually, proliferation of cancer cells is faster than the growth of capillaries, resulting in inadequate perfusion and hypoxia within the tumor. Activation of HIF-1 $\alpha$ in the hypoxic tumor shifts the energy metabolism from oxidative phosphorylation to anaerobic glycolysis, saves oxygen, and avoids massive cell death. As a result of the elevated glucose metabolism, FDG-PET serves as a useful imaging modality for cancer patients.

\subsubsection{Conclusion}

Dominant inflammatory cells activated and responsible for FDG uptake in inflammatory diseases may differ depending upon the disease. All inflammatory cells and cancer cells seem to share the same molecular mechanism of elevated glucose metabolism, lending support to the idea of the usefulness of FDGPET/CT also for the evaluation of inflammatory diseases, besides cancer. Although a guideline [22] has been proposed, use of FDGPET/CT for inflammatory diseases is still limited. I hope that this book will help spread knowledge about the application of FDGPET/CT for inflammatory diseases to improve patient management, representing an advance in the field of medicine.

\section{Acknowledgement}

I would like to thank Roko Kubota, PhD, and Susumu Yamada, $\mathrm{MD}, \mathrm{PhD}$, for their comments and contribution to our research works that reviewed here.

\subsection{FDG PET Imaging of Inflammation in Atherosclerosis}

\section{Mikako Ogawa}

Abstract A vulnerable plaque is characterized by a large lipid-rich atheromatous core, a thin fibrous cap, and infiltration by inflammatory cells (e.g., macrophages). The rupture of high-risk, vulnerable plaques can cause thrombosis, the main cause of acute myocardial infarction and stroke. Thus, the detection of vulnerable plaques is clinically important for risk stratification and early administration of treatment. Macrophages play a central role in the destabilization of atherosclerotic lesions. Macrophages are metabolically active; therefore, these plaques can be detected by FDG-PET. Thus far, numerous clinical and basic research studies have been performed to investigate the effectiveness of FDGPET for the imaging of vulnerable plaques. In addition, it was revealed that the foam cell formation of macrophages affects the accumulation of FDG. Notably, FDG accumulates in pro-atherogenic M1 macrophages rather than anti-inflammatory M2 macrophages. Therefore, assessing the effect of drugs in individual patients is important to monitor the therapeutic effect. Moreover, numerous studies have shown that FDG-PET is useful in evaluating the therapeutic effect of drugs.

Keywords: Macrophage, Atherosclerosis, Vulnerable plaque, FDG-PET

\subsubsection{Introduction}

Atherosclerotic plaques are classified into two types: stable and vulnerable. Vulnerable plaques are easy to rupture and may cause stroke and heart attack. Therefore, the prompt detection and treatment of vulnerable plaques, prior to the manifestation of symptoms, is of crucial importance. Vulnerable plaques are characterized by a lipid-rich atheromatous core, which is infiltrated by macrophages. There is a correlation between the number of infiltrating macrophages and the severity of symptoms in acute myocardial infarction $[23,24]$. In contrast, infiltration by macrophages is rarely observed in stable plaques. 
Macrophages are active in energy metabolism. Therefore, FDG-PET is a promising tool for the detection of vulnerable plaques, depending on the extent of macrophage infiltration.

\subsubsection{Uptake of FDG in Vulnerable Plaques}

Thus far, numerous clinical and nonclinical studies have investigated the use of FDG for the imaging of vulnerable plaques. In 1994, Kubota et al. demonstrated that FDG accumulated more in macrophages than in tumor cells [25]. Therefore, it is assumed that vulnerable plaques can be detected depending on the rate of macrophage infiltration in the plaque. In the early 2000s, several clinical studies suggested that it is possible to detect atherosclerosis, according to the level of inflammation [26-28]. Notably, through histological analysis of symptomatic carotid artery plaques, Rudd et al. reported the co-localization of $\left[{ }^{3} \mathrm{H}\right] \mathrm{FDG}$ and macrophages [27].

The investigators examined the relationship between the degree of macrophage infiltration and accumulation of FDG in Watanabe heritable hyperlipidemic (WHHL) rabbits-an arteriosclerotic animal model-, and explored the possibility of detection of vulnerable plaques using FDG-PET [29]. The atherosclerotic plaques were detected by FDG-PET (Fig. 1.5). In WHHL rabbits, thickening of the intima and infiltration by macrophages was observed in all individuals. We examined the degree of macrophage filtration via histological analysis. The results showed that the FDG uptake and number of macrophages in the atherosclerotic lesions were strongly correlated (Fig. 1.6). However, there was no correlation between the degree of macrophage infiltration and that of intimal thickening. These results suggested that macrophages are responsible for the accumulation of FDG in atherosclerotic lesions, and vulnerable plaques may be detected through FDG-PET.

Studies in humans have shown that the uptake of FDG into vulnerable plaques correlates with the infiltration by macrophages [30, 31]. Immunohistochemical staining of macrophage marker CD68 revealed a strong correlation with FDG accumulation. It has also been reported that the incorporation of Matrix Metalloproteinase-1-an enzyme involved in the destabilization of plaques-and FDG are strongly related [32]. Tahara et al. reported correlations between the accumulation of FDG and waist circumference, hypertension, insulin resistance, lowering of high-density lipoprotein cholesterol, etc. [33]. A recent study showed a correlation of FDG accumulation with C-reactive protein [34]. The results of these studies indicate the prospect for the use of FDG-PET in arteriosclerosis-related diseases.

\subsubsection{Foam Cell Formation and FDG Uptake}

Foam cell formation is responsible for the vulnerability of plaques. Macrophages are recruited from blood monocytes that enter through the endothelium (Fig. 1.7). Scavenger receptors on macrophages mediate the uptake of oxidized low-density lipoprotein (LDL) and cause the accumulation of LDL-derived cholesterol and foam cell formation. Foam cells release several proteases and cytokines, which lead to rupture of the plaques. We evaluated the effects of foam cell formation on the uptake of FDG [35]. Macrophages were isolated from the peritoneum cavity of mice, and foam cells
Fig. 1.5 Fused PET-CT images of WHHL and control rabbits. The WHHL rabbit is an animal model of atherosclerosis. The pink arrowheads show the aortas. The aorta was clearly imaged using FDG in WHHL rabbits
I

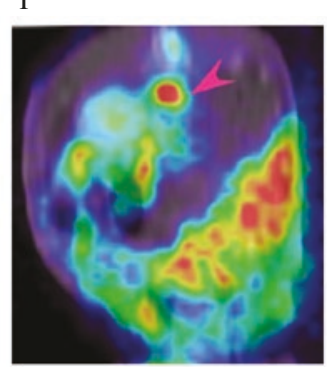

II

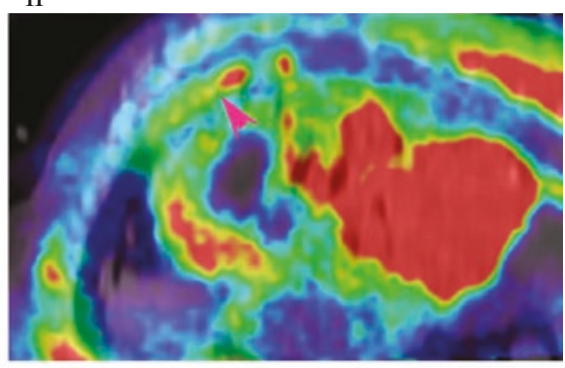

I

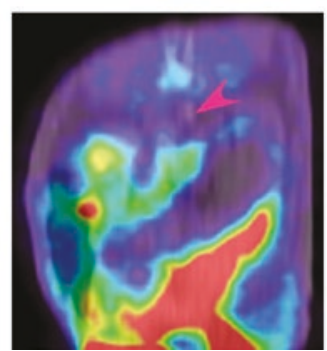

II

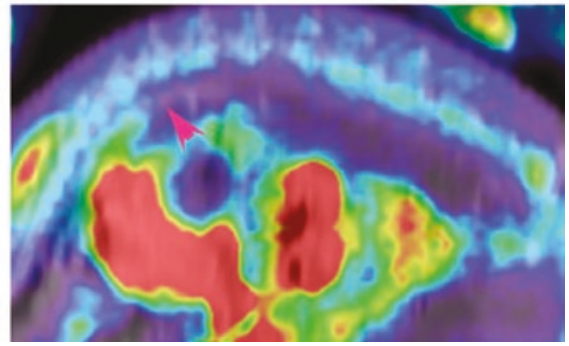


Fig. 1.6 Correlation between the uptake of FDG and the number of macrophages in the aortic segments of WHHL rabbits. The uptake of FDG was well correlated with the degree of macrophage infiltration. DUR differential uptake ratio

Fig. 1.7 Scheme of the development of vulnerable atherosclerotic plaques. Monocytes differentiate into macrophages, and foam cells are formed through stimulation of a scavenger receptor by oxidized low-density lipoprotein (ox-LDL)

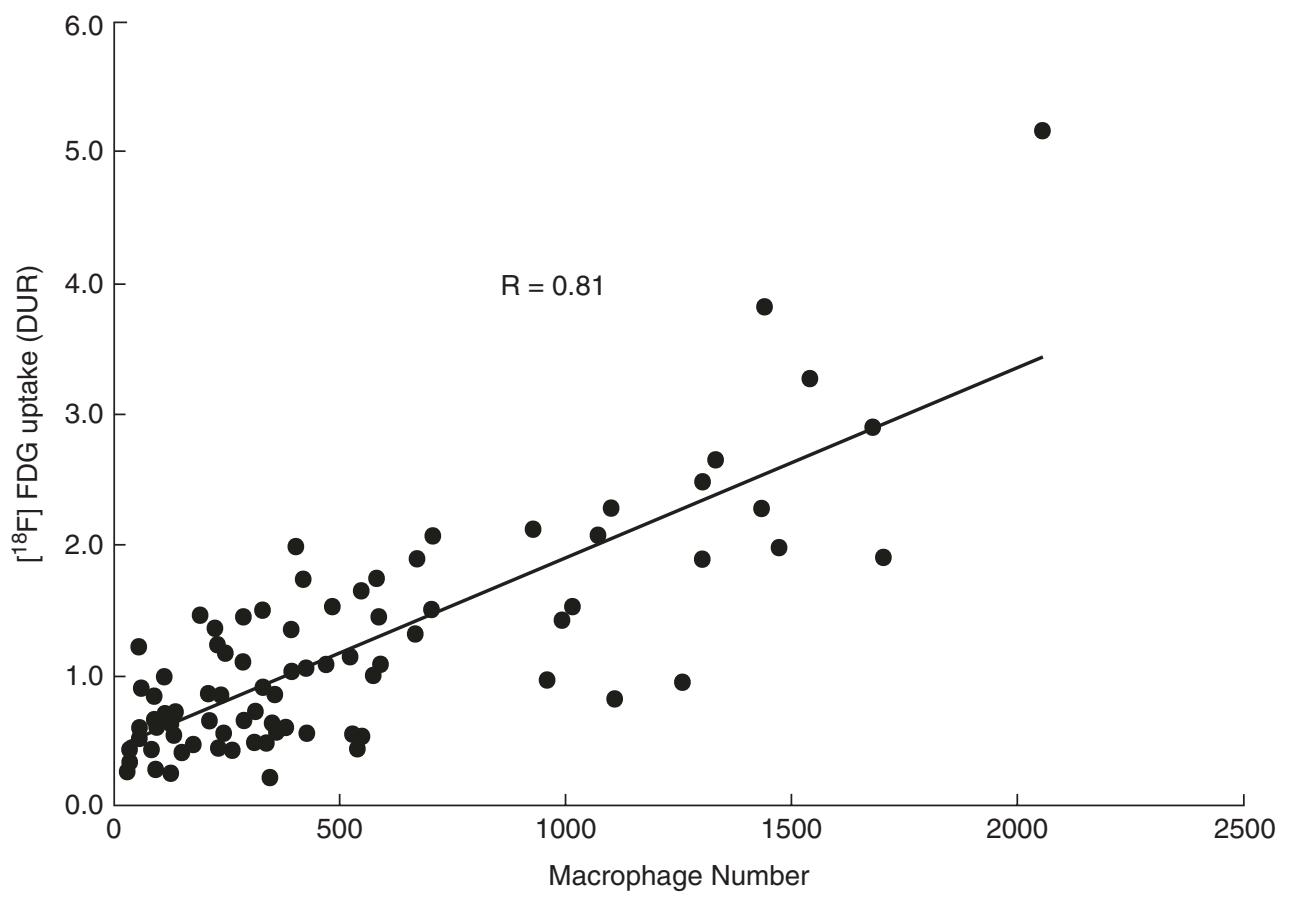

$$
\text { DUR }=\frac{\text { tissue activity }(\mathrm{Bq}) / \text { tissue weight }(\mathrm{mg})}{\text { injected } \mathrm{Rl} \text { activity }(\mathrm{Bq}) / \text { animal body weight }(\mathrm{kg})}
$$

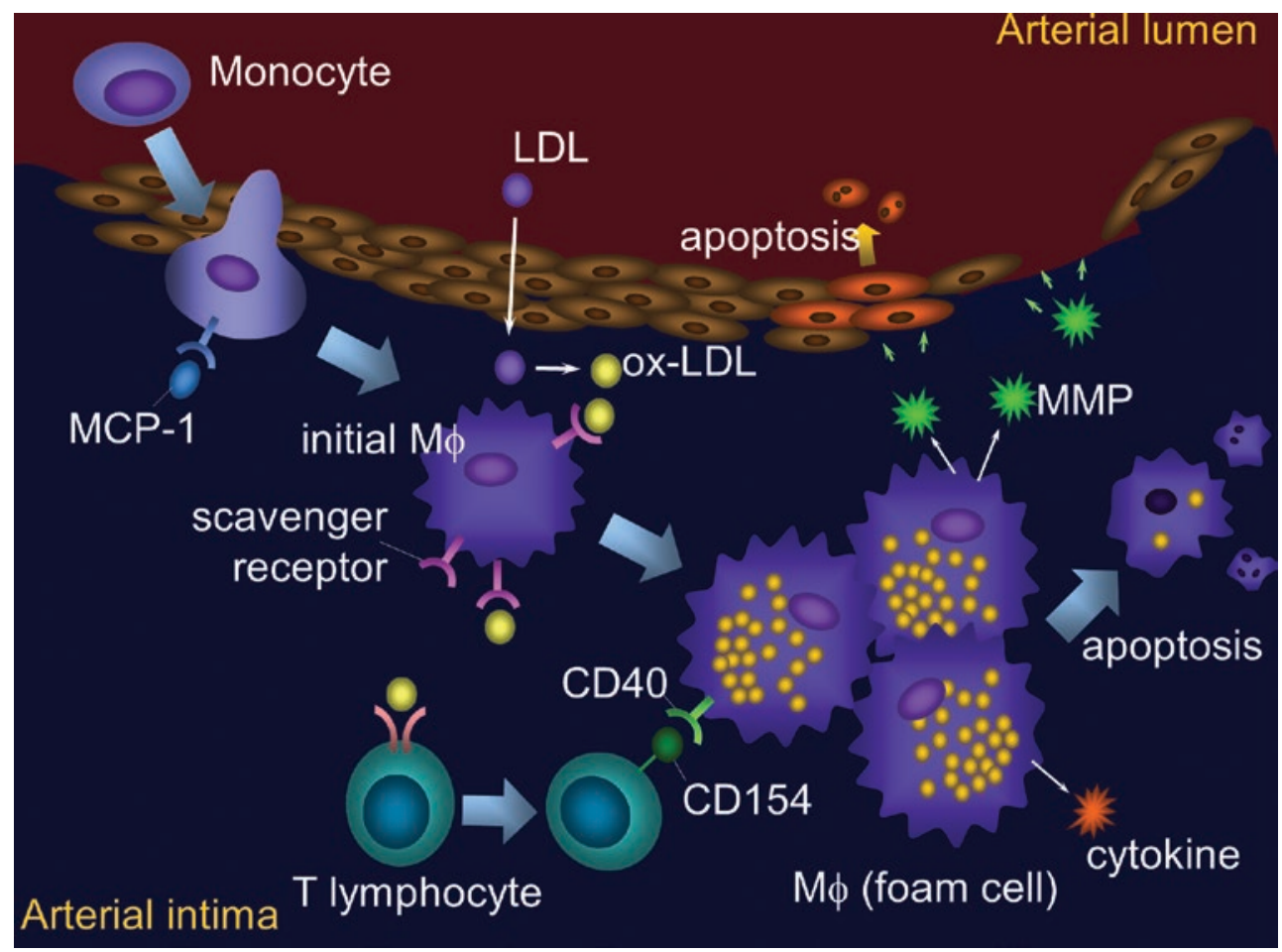

were induced by acetylated LDL. The accumulation of FDG was increased via foam cell formation. However, the uptake was decreased to the level of control, following the complete differentiation into foam cells. The activity of hexokinase was changed in parallel with the uptake of FDG, i.e., higher activity was observed $24 \mathrm{~h}$ after acetylated-LDL loading versus the control condition. Of note, the observed changes in glucose-6-phosphatase activity and glucose transporter 1 
expression were not parallel to the uptake of FDG. This suggests that FDG-PET detects the early stage of foam cell formation in atherosclerosis, depending on the activity of hexokinase. Moreover, using microautoradiography (ARG) in WHHL rabbits, it has been shown that the degree of infiltration by foamy macrophages in atherosclerotic lesions relates to the uptake of $\left[{ }^{3} \mathrm{H}\right] \mathrm{FDG}[36]$.

\subsubsection{Polarization of Macrophages and Uptake of FDG}

Recent studies have described that polarization of macrophages affects the development of atherosclerosis and rupture of plaques. Classically activated M1 macrophages are considered to possess the most proatherogenic phenotype and promote the destabilization of atherosclerotic plaques. In contrast, alternatively activated M2 macrophages possess anti-inflammatory properties and stimulate reparative processes, which lead to the stabilization of atherosclerotic plaques [37, 38] (Fig. 1.8). Therefore, the detection of M1 macrophages may assist in predicting cardiovascular events with greater accuracy. In our investigation, M1 macrophages showed a 2.6-fold increased uptake of $\left[{ }^{3} \mathrm{H}\right]$ FDG versus M2 macrophages [39]. Glucose transporter (GLUT)-1 and GLUT-3, which are major isoforms of a glucose transporter in macrophages, were significantly upregulated in M1 macrophages versus M2 macrophages.

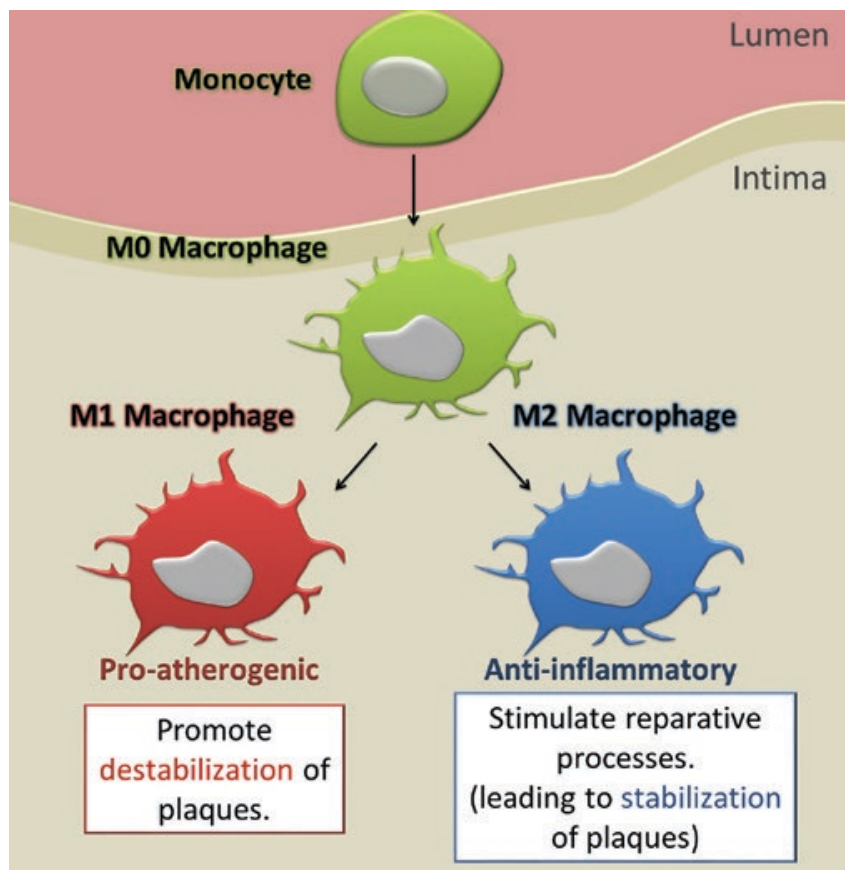

Fig. 1.8 Polarization of macrophages in vulnerable atherosclerotic plaques. M1 macrophages promote the destabilization of plaques, whereas M2 macrophages lead to the stabilization of plaques
Furthermore, hexokinases 1 and 2 were significantly upregulated in M1 macrophages versus M2 macrophages. In contrast, the expression of glucose-6-phosphatase was significantly downregulated in M1 macrophages versus M2 macrophages. In vivo studies showed accumulation of $\left[{ }^{14} \mathrm{C}\right] \mathrm{FDG}$ in the plaques and elevation of M1 markers (i.e., inducible nitric oxide synthase, interleukin- $1 \mathrm{~b}$, and chemokine receptor 7$)$. These results suggest that $\left[{ }^{18} \mathrm{~F}\right] \mathrm{FDG}-$ PET dominantly visualizes the M1 macrophage-infiltrated areas.

\subsubsection{Monitoring of the Therapeutic Effect}

To date, numerous drugs have been developed for the treatment of atherosclerosis. The therapeutic effects of these agents are usually monitored by determining the levels of lipids in the blood. However, lipid-lowering therapy does not always lead to the stabilization of vulnerable plaques. Several statins may effectively reduce the levels of cholesterol in the plasma; however, they do not decrease the degree of macrophage infiltration $[40,41]$. Thus, merely monitoring the levels of lipids in the plasma is not sufficient to determine the therapeutic effect of drugs. Macrophage infiltration plays an essential role in the rupture of plaques. Therefore, the administration of pharmacological therapy that reduces macrophage infiltration is required to stabilize the vulnerable plaques. Considering the individual differences in the stabilization of plaques induced by such drugs, monitoring the therapeutic effect in each individual plaque is important to accurately assess the effect.

Using probucol as a therapeutic drug in WHHL rabbits, we investigated the usefulness of FDG-PET for the monitoring of therapies that target vascular inflammation. In a number of animal studies, the lipid-lowering effect of probucol was moderate, and the drug did not decrease the levels of cholesterol in the plasma [42, 43]. However, probucol can reduce the macrophage-rich plaques even in advanced atherosclerotic lesions [43]. In the present study, the uptake of FDG was significantly decreased in the probucol group versus the pretreatment period (Fig. 1.9). However, there was no difference in the levels of cholesterol in the plasma between the probucol and control groups [44]. A large number of macrophages was observed at the initiation of the study. Nevertheless, treatment with probucol for 6 months resulted in diminished macrophage infiltration (Fig. 1.10). Notably, the ratio of the intima to the whole cross-sectional area was not affected by treatment with probucol. Hence, the observed decrease in the uptake of FDG was attributed to the decrease in the number of infiltrating macrophages. Collectively, these studies showed that the therapeutic effect of probucol was successfully monitored by FDG-PET independently of the cholesterol-lowering effect. 
Fig. 1.9 Time course of the uptake of FDG in the aortas of WHHL rabbits over the treatment period. After 3 months of treatment with probucol, the uptake of FDG (SUV) decreased in all treated rabbits. In contrast, the SUV remained constant or increased in control rabbits

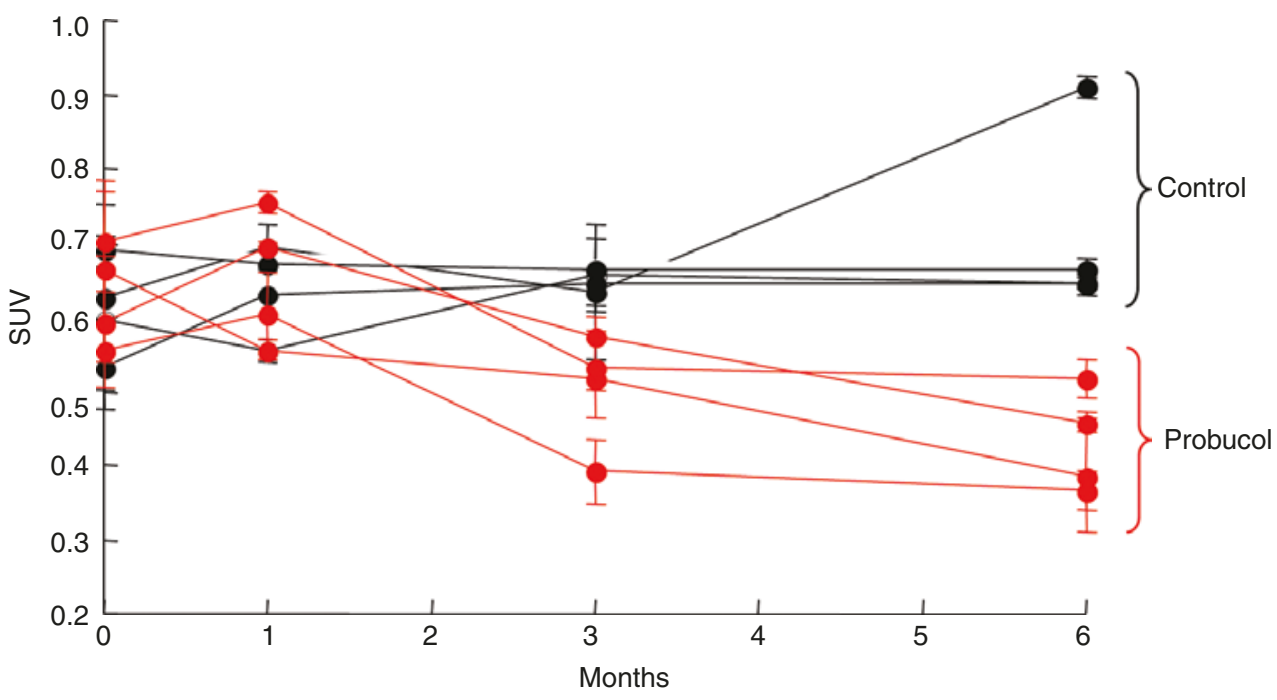

Afere 6-months of investigation Control

Probucol

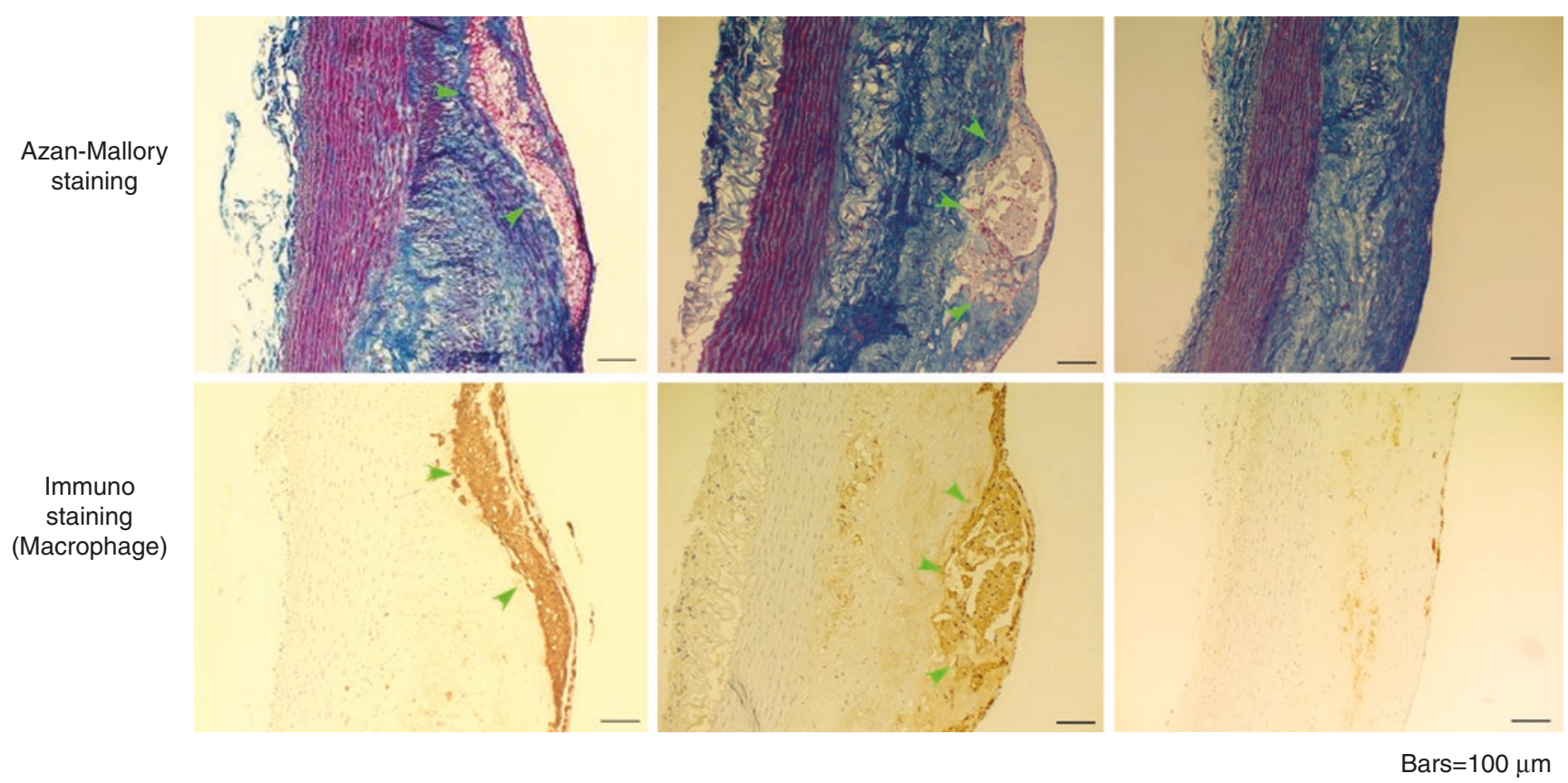

Fig. 1.10 Typical histological images of Azan-Mallory-stained and macrophage immunohistochemically stained slices. Green arrow heads indicate macrophages. Treatment with probucol resulted in diminished macrophage infiltration

These results demonstrated the usefulness of FDG-PET for drug development, and numerous clinical studies have been performed [45-49]. Tahara et al. conducted one of the initial clinical trials, showing that the therapeutic effect of simvastatin was successfully monitored by FDG-PET [50]. In 2011, the results of the first clinical multicenter study investigating the efficacy of dalcetrapib against atherosclerotic disease via a novel noninvasive multimodality imaging technique (dal-PLAQUE) were reported. Of note, FDG-PET was used to evaluate the level of vascular inflammation [51].
Moreover, in the dal-PLAQUE study, the relationship between serum inflammatory biomarkers and plaque inflammation was also assessed using FDG-PET [52].

\subsubsection{Comparison with Other PET Tracers}

Recently, several PET imaging tracers-apart from FDGhave been reported for the imaging of vulnerable plaques. For example, $\left[{ }^{11} \mathrm{C}\right]$ choline and $\left[{ }^{18} \mathrm{~F}\right]$ fluoromethylcholine are 
used for the detection of increased cell proliferation in the plaques $[53,54]$. In addition, $\left[{ }^{11} \mathrm{C}\right] \mathrm{PK} 11195$ is used to detect translocator protein (TSPO) on macrophages [55, 56]. Moreover, $\left[{ }^{68} \mathrm{Ga}\right]$ DOTATOC and $\left[{ }^{68} \mathrm{Ga}\right]$ DOTATATE are used to determine the expression of somatostatin receptor subtype 2 on macrophages [57, 58]. Furthermore, $\left[{ }^{18} \mathrm{~F}\right] \mathrm{FMISO}$ is used for the detection of hypoxia around the neovascularization area near the plaques [59], while $\left[{ }^{18} \mathrm{~F}\right] \mathrm{NaF}$ is used for the detection of calcification [60-63]. Recently, the $\beta$-amyloid imaging agent $\left[{ }^{18} \mathrm{~F}\right]$ Flutemetamol showed specific accumulation in human carotid plaques, especially in amyloid betapositive areas [64].

Among these tracers, $\left[{ }^{18} \mathrm{~F}\right] \mathrm{NaF}$ is considered a promising probe for the detection of atherosclerosis, and several comparison studies with FDG have been performed. The results of these studies showed that the area of accumulation differs between FDG and $\left[{ }^{18} \mathrm{~F}\right] \mathrm{NaF}$ [65-67]. The accumulation of $\left[{ }^{18} \mathrm{~F}\right] \mathrm{NaF}$ in atherosclerotic plaques depends on the level of calcification [68]. The $\left[{ }^{18} \mathrm{~F}\right] \mathrm{NaF}$-positive area does not completely match with the CT-positive area, and it is thought that $\left[{ }^{18} \mathrm{~F}\right] \mathrm{NaF}$ accumulates in the early stage of calcification (i.e., microcalcification) $[60,69]$. Since target molecules are different among these tracers, comparison study should be important to elucidate the specificity of these tracers for the different stages of atherosclerosis progression. This may assist in understanding the mechanism of plaque progression and provide important insight into therapy aimed toward the stabilization of plaques.

\subsection{PET Imaging in Animal Model of Neuroinflammation 1}

Bin Ji

\begin{abstract}
Neuroinflammation is a general event in acute and chronic neurodegenerative disorders. Based on the critical role of neuroinflammation characterized by glial activation in neuropathogenesis, in vivo imaging with positron emission tomography (PET) is required in clinical and preclinical studies for the purposes of elucidation of pathogenesis and novel treatment development, because it is commonly available in human and experimental animal models. As a most widely used imaging biomarker for neuroinflammation, $18 \mathrm{kDa}$ translocator protein (TSPO) imaging has been performed in a large number clinical and preclinical studies. Neuropathologyassociated TSPO induction has been generally detected in various neurodegenerative animal models with acute and chronic neuroinflammation. However, studies with human subjects showed confusing results likely due to ineffectiveness of the tracers used or impediment of non-microglial TSPO expression in human diseased brains. Based on the
\end{abstract}

above reasons, recently, alternative molecular targets for microglia imaging instead of TSPO have been proposed. Colony-stimulating factor 1 receptor (CSF1R) is a promising candidate because of its highly specific expression in microglia in the central nervous system (CNS). Purinergic receptors $\mathrm{P} 2 \mathrm{X} 7 \mathrm{R}$ and $\mathrm{P} 2 \mathrm{Y} 12 \mathrm{R}$ have been proposed as imaging biomarkers of M1 and M2 phenotype microglia, respectively. Several PET tracers for these non-TSPO biomarkers have been developed, and some of them showed positive results in animal models. However, more exploratory work is needed for further application in human subjects.

Keywords: Neurodegenerative disorders, Neuroinflammation, Glial activation, In vivo imaging, $18 \mathrm{kDa}$ translocator (TSPO), Colony-stimulating factor 1 receptor (CSF1R)

\subsubsection{Introduction}

Neuroinflammation is characterized by glial activation, which would lead to an increase in the inflammatory factor level in the CNS. Microglia are a collection of glial cells that act as the first and main form of active immune defense in the CNS. Accordingly, its activation is considered to trigger neuroinflammation, and PET tracers bound to active microglia are used for imaging of neuroinflammation. Therefore, molecules exclusively expressed in microglia have the potential to be molecular targets for neuroinflammation. Because imaging with PET is a commonly available technology for human subjects and experimental animal models, numerous PET tracers have been developed for visualization of microglia in living brains. Moreover, increasing evidence has indicated that microglial activation is heterogeneous, and can be categorized into two opposite types: pro-inflammatory (M1) and anti-inflammatory (M2) microglial phenotypes. PET imaging tracers that bind to either general or phenotypespecific microglia are required for studies of neurodegenerative disorders. PET imaging in animal models is an indispensable step for the development and clinical application of PET tracers, as it will provide predictive evidence for tracer utility in human subjects. More importantly, it can easily provide a direct comparison between PET images and histopathology, which is usually difficult in human studies. PET imaging in animal models would supplement the weak points of human studies and provide interpretation for imaging in human subjects.

\subsubsection{Current Standard of Neuroinflammation Imaging}

$18 \mathrm{kDa}$ Translocator protein (TSPO) is a housekeeping protein in microglia and is greatly induced in active microglia in 
response to neural injury. PET tracers with high affinity for TSPO are the most widely used for neuroinflammation imaging. Tracers have been developed for TSPO imaging for several decades, including typical first-generation tracer ${ }^{11} \mathrm{C}-\mathrm{PK} 11195$ and second-generation tracer ${ }^{11} \mathrm{C}$-DAA1106 [70]. Major parts of these tracers enable the visualization of microglial activation in acute neuroinflammation models triggered by acute events like traumatic brain injury, ischemia, and excitotoxic damages. A longitudinal PET imaging study has shown a continuing increased TSPO PET tracer uptake in a mouse model of mesial temporal lobe epilepsy induced by unilaterally intracranial kainic acid injection. The radioactivity uptake reached a peak at 7 days, mostly related to microglial activation. After 14 days, reactive astrocytes provided a major binding site for TSPO tracer [71]. The finding of phase-dependent TSPO expression in subtypes of glial cells might contribute to the identification of optimal treatment windows in further clinical studies [71]. Chronic neuroinflammation is usually observed in many chronic neurodegenerative disorders such as Alzheimer's disease (AD), amyotrophic lateral sclerosis (ALS), and non-AD tauopathies. Most animal models of chronic neurodegenerative disorders are genetically modified mouse models. Authors have clearly demonstrated glial activation in response to accumulation of amyloid aggregates and treatment with the aid of TSPO-PET, indicating the utility of neuroinflammation imaging in monitoring of pathogenesis (Fig. 1.11). Amyloid accumulation is the earliest pathological change in the brain with $\mathrm{AD}$, followed by tau aggregation and glial activation. Glial activation is predominantly concentrated around neuritic plaques, not diffuse plaques [72]. More clinical studies have reported increased TSPO tracer accumulation in temporoparietal, entorhinal, and cingulate cortex rather than frontal cortex with rich diffuse plaques and poor neuritic plaques, partially supporting such observation, while a considerable number of studies failed to detect an increase in TSPO

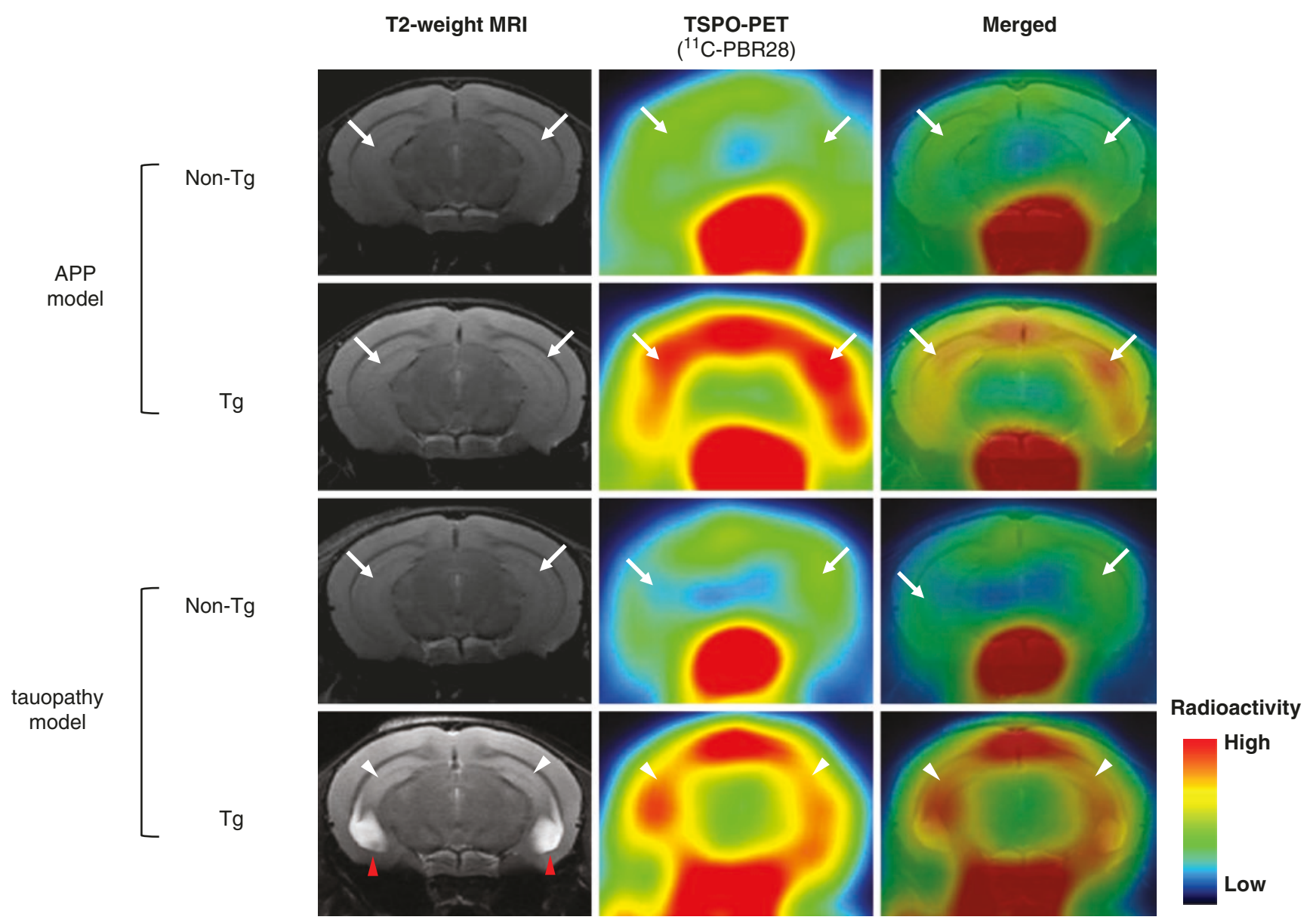

Fig. 1.11 TSPO-PET in AD mouse models mimicking amyloid and tau pathologies. Representative merged coronal brain images of T2-weighted MR, TSPO-PET and merged images showed that tracer binding was greatly increased in transgenic mice (Tg) of APP and tauopathy models mimicking amyloid (22-month-old) and tau (12-month-old) pathologies, respectively, compared with respective age-matched non-transgenic littermate mice (non-Tg). No obvious brain atrophy was observed in hippocampi of APP model as well as non-Tg mice (white arrows), while tauopathy model showed overtly atrophic hippocampi (white arrowheads). Red arrowheads indicated enlarged ventricles due to the hippocampal atrophy. Unpublished data 
expression level [73]. The amyloid plaques accumulated in the amyloid precursor protein (APP) model are dense-core plaques, around which there are abundant activated glial cells and TSPO expression, indicating a similar glial response between neuritic and dense core plaques. However, immunohistochemical analysis showed that active astrocytes have provided the majority of binding sites for TSPO tracer in the APP model [74], while microglia around neuritic plaques expressed abundant TSPO [75]. This distinction between species concerning glial characteristics might be attributable to neurodegenerative tau pathologies, which exist in AD but are uncommon in the APP model. In vivo TSPO imaging also demonstrated increased TSPO expression accompanied by tau-related atrophic brain in another model of AD mimicking the tau-related pathologies (Fig. 1.11) [72, 76]. Considering that predominant microglial TSPO expression and age-dependent brain atrophy in these tauopathy models mimicking tau-related pathologies are consistent with the condition in AD patient brains, neuroinflammation imaging in tauopathy models might well reflect the actual situation of pathologies in human subjects. Based on the fact that in vivo imaging for amyloid and tau, as well as TSPO have been available for clinical and preclinical studies, multiple tracer imaging enables the clarification of the mutual relationship between these AD-related pathologies. For example, longitudinal in vivo imagings with TSPO-PET $\left({ }^{18} \mathrm{~F}\right.$-FEDAA1106) and amyloid-PET $\left({ }^{11} \mathrm{C}-\mathrm{PiB}\right)$ have captured the increased level of neuroinflammation triggered by $\mathrm{A} \beta$ immunotherapy, which greatly reduced amyloid accumulation in an identical APP model over the course of treatment (Fig. 1.12) [77]. TSPO-PET in combination with tau-PET $\left({ }^{11} \mathrm{C}-\mathrm{PBB} 3\right)$ in two tauopathy models showed that neuroinflammation was induced by two different tau aggregates (PBB3-positive and -negative) and thereby accelerated tau-mediated neuron damage via a distinct molecular mechanism [78]. These studies provide experimental evidence for neuroinflammation-targeting therapeutic strategy. Similarly, TSPO tracer signals were also increased in lesioned brain regions in a

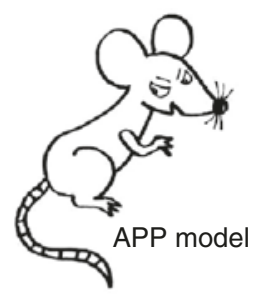

b
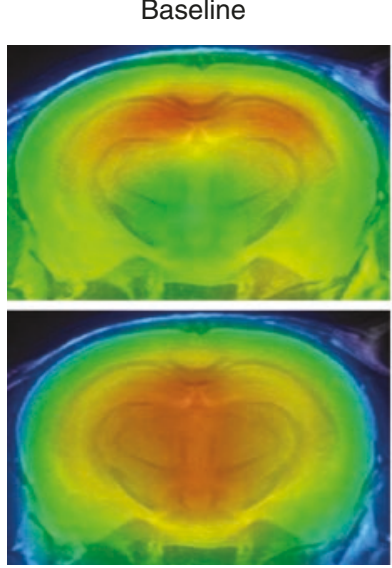

$A \beta$

immunotherapy

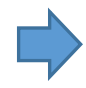

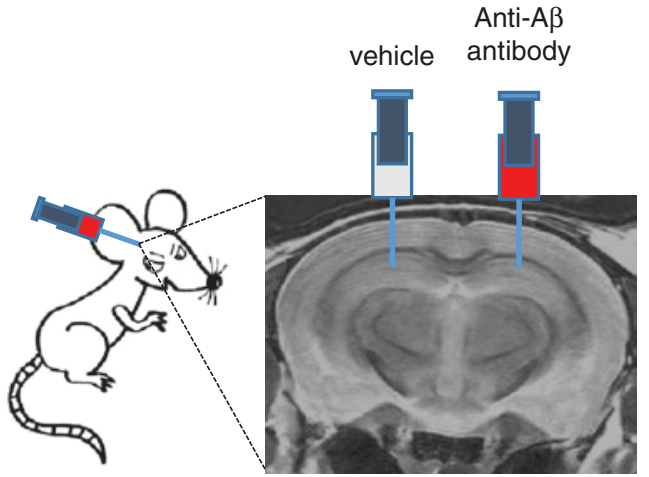

$A \beta$ immunotherapy

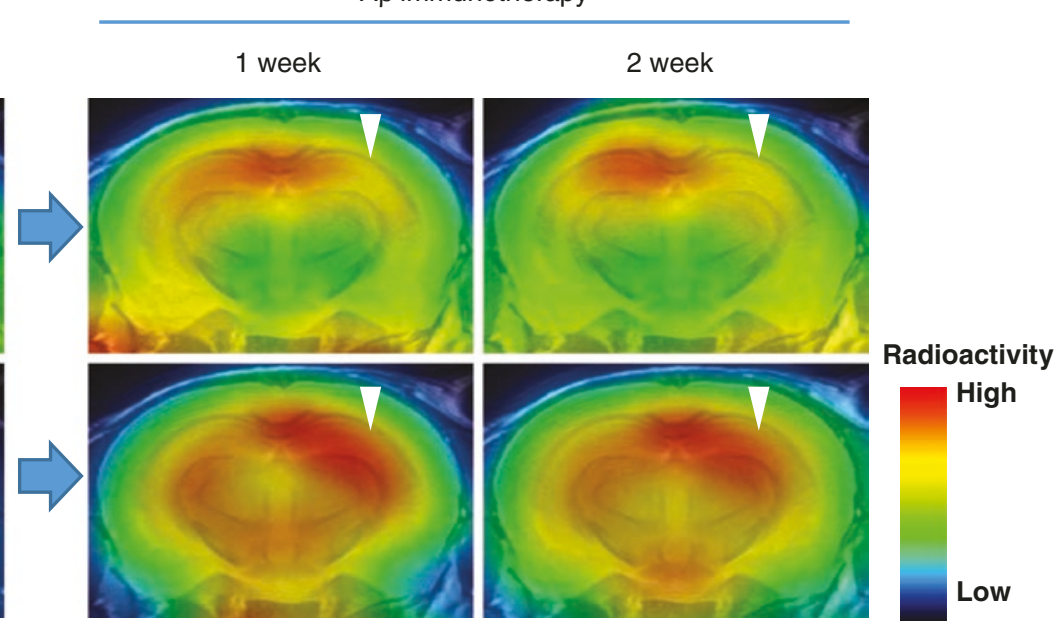

Fig. 1.12 Monitoring of anti-A $\beta$ effect and neuroinflammation in amyloid immunotherapy. (a) A $\beta$ immunotherapy was performed in APP model mice by intracranial injection of a therapeutic anti-A $\beta$ antibody into the right hippocampus, and equivalent amount of vehicle was injected into the contralateral hippocampus as control. (b) APP model mice received repeated PET scans before (baseline) and after (1- and 2-week) treatment. Representative images of amyloid- and TSPO-PET merged with MRI brain template in an APP mouse showed that A $\beta$ immunotherapy greatly reduced amyloid accumulation (amyloid-PET) and induced an increase in TSPO expression (TSPO-PET) around the region treated by antibody (white arrowheads), but not vehicle. (Data were modified from ref. [3]) 
mouse model of ALS, being consistent with microglial immunostaining [79]. In contrast, the results from Parkinson's disease (PD) animal models look more complicated. PD animal models are relatively easy to prepare by treating the animals with neurotoxins such as methamphetamine and 6-hydroxydompaine (6-OHDA). Methamphetamine-induced degeneration of dopaminergic neuron terminals did not trigger TSPO-positive gliosis [74], while 6-OHDA induced degeneration of dopaminergic neurons in either striatum or substantia nigra and increased the TSPO tracer signal [80]. Interestingly, most of the clinical PET studies found no increase in the level of TSPO expression in the striatal regions of PD patients $[73,81,82]$ despite severe dopaminergic degeneration in these regions, supporting the proposal that TSPO imaging is inapplicable to monitoring the degeneration of nonmyelinated nerve [74].

Quantitative PET studies require that the input function, representing cumulative availability of authentic radiotracer in arterial plasma, is measured in case of the absence of ideal reference tissue. Because of the constitutive expression of TSPO in the whole brain, blood collection is needed for calculating the binding potential. However, this usually faces technical difficulties in the actual operation for the small mouse body. So the brain regions without pathological changes, such as cerebellum in rTg4510 mouse [76, 78] or striatum in PS19 mouse [72], are used as reference tissue when reference-tissue models are used. Although it will lead to underestimation of the binding potential due to constitutive low-level TSPO expression in reference regions, such analysis can provide relatively stable results compared to the percentage of injection dose, which is greatly affected by body weight, metabolic capacity, or other factors. Another issue is a concern for the partial volume effect due to the small volume of the region of interesting (ROI) in the case of mouse. This makes it difficult to correctly measure the radioactivity in certain subregions of mouse brain. The use of rat and nonhuman primate models is an alternative, but selectable neurodegenerative models are limited compared to mouse, as most models with pathologies of chronic neurodegenerative disorders, such as AD, ALS, and non-AD tauopathies [83, 84], are genetically modified murine models. Despite the fact that TSPO-PET successfully captured neuroinflammation in various animal models with acute and chronic neuroinflammation, the results in patients with chronic neuroinflammation seemed more complicated [73, 81]. One of the reasons is that a TSPO polymorphism (rs6971) greatly influences the affinity of a large majority of TSPO tracers in a tracer-dependent manner [85]. Another possible reason is the differing cellular distribution of TSPO expression. Recent studies have shown that TSPO expression is not limited to microglia. Other brain components, for example, astrocytes and vascular endothelial cells, also express TSPO. Some studies have demonstrated dominant induction of TSPO expression in active astrocytes in a neuropathological type-dependent and phase-dependent manner $[71,74]$. Vascular expression of TSPO is also a factor that disturbs microglial TSPO imaging. Although no study has quantitatively measured the influence of vascular TSPO on tracer binding, inclusion of the additional vascular component in the modified reference-tissue model amplified the binding potential in AD more than in control by decreasing tracer binding to the vasculature in the disease cohort or was better correlated to brain TSPO mRNA [86, 87]. As few studies have focused on changes in vascular TSPO expression in diseased brains, determining which is responsible for the changes in TSPO tracer binding in diseased brains is a complicated issue. Further studies in combination with postmortem analyses or microglial TSPO-specific or vascular TSPO-specific knockout animals are desirable.

\subsubsection{Alternative Molecular Targets for Microglia Imaging}

CSF1R is a novel imaging biomarker for microglia by its exclusive expression in microglia in the CNS [88]. Recently, Horti et al. reported a newly developed PET tracer, ${ }^{11} \mathrm{C}-\mathrm{CPPC}$, for CSF1R imaging. ${ }^{11} \mathrm{C}-\mathrm{CPPC}$ showed high initial brain uptake and rapid washout from brain in normal rodent brain, and increased accumulation in inflammatory brains of rodent and nonhuman primate models including LPS-injection and AD mouse models. In vitro autoradiogram showed higher binding in postmortem brains with AD compared to healthy control [88]. These results support the concept that CSF1R is a suitable molecular target for microglia imaging. However, ${ }^{11} \mathrm{C}-\mathrm{CPPC}$ binding is increased in whole brain regions, and is not limited to regions with lesions as exemplified by cerebellum in AD model and intracranial LPS injection model, and immunohistochemistry for active microglia and CSF1R expression to support imaging results was not provided. Another reported CSF1R imaging tracer, ${ }^{11} \mathrm{C}-\mathrm{AZ} 683$, showed high affinity for CSF1R ( $\mathrm{Ki}=8 \mathrm{nM})$ and $>250$-fold selectivity over other kinases tested, but low brain uptake in rodents and nonhuman primates limited its utility in living brains [89]. Further examinations and improvements will be required for their clinical application. It is still unclear which microglia phenotypes predominantly express CSF1R because of the lack of data from immunohistochemical and biochemical analyses of the various neurodegenerative models.

$\mathrm{P} 2 \mathrm{X} 7 \mathrm{R}$ and P2Y12R were considered to be predominantly expressed in M1 and M2 microglia phenotypes, respectively [90]. In vitro autoradiographic analysis with ${ }^{11} \mathrm{C}-\mathrm{SMW} 139$ showed high specific binding to viral vectormediated human $\mathrm{P} 2 \mathrm{X} 7 \mathrm{R}$ expressed in rat brain, but no increase in binding was detected in postmortem brain with 
AD compared to healthy control [91]. Specific binding of ${ }^{11} \mathrm{C}$-GSK1482160 is also detectable in P2X7Roverexpressing samples under in vitro condition [92]. However, more evidence is required for both of the above two P2X7R tracers to prove their utilities in the living inflammatory brain. In vivo imaging with ${ }^{18} \mathrm{~F}-\mathrm{JNJ}-64413739$ with high affinity and selectivity for P2X7R showed significant increase in tracer accumulation in the living LPSinduced neuroinflammatory brain, indicating its leading status among the current P2X7R tracers, while further work is needed to verify its utility in other types of neuroinflammation [93-95]. Villa et al. recently developed a carbon11-labeled P2Y12R-binding compound. In vitro and ex vivo experiments with this radioactive compound showed increased binding in brain sections of mice treated with anti-inflammatory stimuli and decreased binding to brain sections of a murine stroke model and of a stroke patient [96]. Immunohistochemistry and immunoblotting also supported the viewpoints that $\mathrm{P} 2 \mathrm{Y} 12 \mathrm{R}$ is a biomarker for M2 phenotype microglia $[90,96]$.

\subsection{PET Imaging in Animal Models of Neuroinflammation 2}

Tadashi Watabe

\begin{abstract}
Neuroinflammation is defined as inflammatory responses in the brain and spinal cord, and microglia and astrocytes are the mainly involved cells. Translocator protein (TSPO) has been the major target used in positron emission tomography (PET) to detect neuroinflammation with glial activation. In a preclinical study conducted in small animal models of brain ischemia and traumatic brain injury, a second-generation TSPO tracer $\left[{ }^{18} \mathrm{~F}\right] \mathrm{DPA}-714 \mathrm{PET}$ was used for the evaluation of glial activation; the TSPO uptake was validated by comparison with the immunohistochemical findings of co-staining for TSPO and a microglial/macrophage or astrocyte maker. TSPO expression has also been reported in a model of Alzheimer's disease, where TSPOpositive cells with two opposing roles were observed: neuroprotective astrocytes for reversible neuronal injury and detrimental microglia for irreversible neuronal injury. Recently, new targets have been identified in microglia, namely, cyclooxygenase-1 (COX-1) and purinergic receptor $\mathrm{P} 2 \mathrm{X} 7$, which have been shown to be specifically expressed in the microglia. PET imaging targeting glial cells is a promising modality to characterize and monitor neuroinflammation longitudinally and with quantitative accuracy. The findings of preclinical models need to be confirmed in clinical studies beyond the limitation of animal model and the species differences between rodents and humans.
\end{abstract}

Keywords: Neuroinflammation, Glial activation, Translocator protein, Microglia, Astrocyte

\subsubsection{Introduction}

Neuroinflammation is defined as inflammatory responses in the brain and spinal cord [97]. It is observed in various types of brain diseases, including brain ischemia, traumatic brain injury, neurodegenerative disorders (Alzheimer's disease, Parkinson's disease, etc.), and neuropsychiatric disorders (schizophrenia, autism spectrum disorder, etc.) [98-101]. In the central nervous system, two major glial cells are involved in the process of neuroinflammation, namely, microglia and astrocytes [99]. To evaluate neuroinflammation in vivo, translocator protein (TSPO) has been the major target used in PET for the detection of neuroinflammation with glial activation [102]. In glial cells, especially microglia, expression of TSPO is minimal in the resting state, but upregulated in the activated state [103]. In previous studies, the first-generation TSPOPET tracer, $\left[{ }^{11} \mathrm{C}\right] \mathrm{PK}-11195$, was mainly used to evaluate glial activation in small animals as well as humans. However, the evaluation using $\left[{ }^{11} \mathrm{C}\right] \mathrm{PK}-11195$ was found to be highly limited by the high nonspecific binding of the tracer $[104,105]$; therefore, over the last 10 years, second-generation TSPOPET tracers, such as $\left[{ }^{11} \mathrm{C}\right] \mathrm{DPA}-713$ and $\left[{ }^{18} \mathrm{~F}\right] \mathrm{DPA}-714$, have been used because of their higher specific binding [104, 105]. Although the binding affinity in humans has been shown to vary due to polymorphism, TSPO PET has been employed as an effective tool to visualize and quantify the degree of neuroinflammation associated with glial activation in preclinical studies conducted using animal models, including rodents [106]. However, attention needs to be paid to the inflammatory cells taking up TSPO, as TSPO expression is not only observed in activated microglia/macrophages but also in reactive astrocytes [107].

\subsubsection{Brain Ischemia Model}

For preclinical studies using small animals, the middle cerebral artery occlusion (MACO) model was frequently used owing to its ease of handling for reperfusion and the abundance of accumulated evidence [108, 109]. Martin et al. evaluated the time-course of TSPO expression in the rat model of MCAO, in which transient focal ischemia was induced by 2-h intraluminal occlusion of the MCA, followed by reperfusion [110]. $\left[{ }^{18} \mathrm{~F}\right] \mathrm{DPA}-714$ PET showed significantly enhanced uptake on the ipsilateral side on days 7, 11, 15 , and 21 after the induction of ischemia, with the peak observed on day 11. This finding corresponded to the immunohistochemical results of TSPO expression in the microglia/macrophages (TSPO + /CD11b-positive cells) in the 
ischemic area. The authors also reported an increase in the number of TSPO + /glial fibrillary acidic protein (GFAP)positive cells in the lesioned area on day 21 , with peaking of the number of such cells on day 30; this latter finding suggested the later involvement of reactive astrocytes showing TSPO expression. These results show that $\left[{ }^{18} \mathrm{~F}\right] \mathrm{DPA}-714$ provides accurate quantitative information about the timecourse of TSPO expression in the glial cells in experimental stroke. Figure 1.13 shows the $\left[{ }^{11} \mathrm{C}\right] \mathrm{DPA}-713$ PET images and results of immunohistochemistry using antibodies for $\mathrm{CD} 11 \mathrm{~b}$ and GFAP in the rat model of MCAO 4 days after transient ischemia for $1 \mathrm{~h}$ followed by reperfusion. The areas of enhanced uptake of $\left[{ }^{11} \mathrm{C}\right] \mathrm{DPA}-713$ correspond to the areas showing high expression of CD11b, namely, areas containing a high number of activated microglia/macrophages. Immunohistochemistry showed reactive astrocytes, represented by cells showing GFAP expression, in the boundary of the ischemic area, which showed a low uptake of $\left[{ }^{11} \mathrm{C}\right]$ DPA-713 on PET images. In regard to the clinical significance of the findings in relation to treatment, Martin A et al. reported that uptake of $\left[{ }^{11} \mathrm{C}\right] \mathrm{DPA}-713$ in the ischemic lesion could be attenuated by administration of minocycline $1 \mathrm{~h}$ after the reperfusion [111]. Thus, TSPO PET can also be used for monitoring the response to immunomodulatory therapy after brain ischemia.

\subsubsection{Traumatic Brain Injury Model}

To evaluate traumatic brain injury (TBI) in small animals, a model of controlled cortical impact with a pneumatic impact device has been used for its high reproducibility and low mortality [112]. We reported two peaks of $\left[{ }^{18} \mathrm{~F}\right] \mathrm{DPA}-714$ uptake in this model, namely, on day 7 in the cortical area and on day 21 in the thalamus after induction of the focal brain injury [99]. The enhanced thalamic uptake was sustained until 14 weeks after induction of the TBI (Fig. 1.14). Immunohistochemical analysis on day 7 revealed TSPO staining mainly co-localized with amoeboid CD11b-positive microglia/macrophages, and weakly with GFAP-positive astrocytes in the cortex; however, TSPO immunoreactivity was scarcely observed in the thalamus. Giemsa staining on day 7 revealed mononuclear cells, such as phagocytic macrophages, in the cortical area, although these cells were not detected in the ipsilateral thalamus. In the macrophage depletion study performed 1 week post-injury, the TSPO uptake was decreased in the cortex, but enhanced in the thalamus, suggesting that the cortical uptake was mainly attributable to uptake by phagocytic macrophages recruited from the bloodstream, while the thalamic uptake was most likely attributable to uptake by resident microglia. Electron microscopy at 4 weeks post-TBI revealed morphologically activated microglia surrounding the degenerated axons and poorly myelinated neurons in the ipsilateral thalamus, indicating activation of the resident microglia and damage to the remaining neurons. TSPO expression was mainly evident in the activated microglia in the thalamus at 6 weeks postinjury. Thus, by using $\left[{ }^{18} \mathrm{~F}\right] \mathrm{DPA}-714$ PET, inflammatory migration could be detected from the cortex to the thalamus after focal brain injury. In addition, we also showed that depletion of myeloid-derived suppressor cells (MDSCs) was associated with enhanced cortical TSPO uptake on day 7 after the induction of focal brain injury, by both immunohistochemistry and cellular phenotype analysis [113]. This finding indicated that MDSCs may have strong immunosuppressive characteristics and limit inflammation and facilitate wound healing and recovery.

\subsubsection{Alzheimer's Disease Model}

Postmortem analyses of the brains of patients with Alzheimer's disease (AD) have revealed neuroinflammatory changes, namely, accumulation of activated microglia and astrocytes, around senile plaques and fibrillary tau lesions [114]. In this study, diffuse plaques in the brains of these patients were enveloped by a small number of Iba-1positive microglia not expressing TSPO, while numerous microglia expressing both Iba-1 and TSPO were found to be in close contact with neuritic plaques. Thus, microglia expressing TSPO were recruited to fibrillar tau inclusions, but not to $\mathrm{A} \beta$ deposits unaccompanied by tau pathology. Jin et al. reported that TSPO expression was observed, mainly in the astrocytes, in amyloid precursor protein 23 (APP23) transgenic ( $\mathrm{Tg}$ ) mice which show minimal neuronal loss, while TSPO-positive microglia were observed in the tangle-like tau lesions of tau Tg mice which show remarkable neuronal loss. They concluded that TSPO-positive astrocytes have protective roles against reversible neuronal injury, while TSPO-positive microglia have detrimental effects for irreversible neuronal injury [115]. We evaluated TSPO expression in the APP23 Tg mice by immunohistochemistry (Fig. 1.15). TSPO immunoreactivity was observed around the amyloid beta $(\mathrm{A} \beta)$ plaques and in reactive astrocytes (GFAP-positive). CD11b-positive microglia were relatively few in number as compared to the number of GFAP-positive astrocytes, consistent with the findings reported by Jin B et al. However, it should be borne in mind that no significant increase in the uptake of $\left[{ }^{18} \mathrm{~F}\right] \mathrm{DPA}-714$ or of the amyloid tracer $\left[{ }^{11} \mathrm{C}\right] \mathrm{PIB}$ was detected by in vivo PET performed prior to the immunohistochemistry in the same mice. Maier $\mathrm{F}$ et al. reported that $\mathrm{A} \beta$ deposition was detectable earlier by histology than by amyloid PET in APP23 Tg mice, indicating the existence of a lower limit of sensitivity for the detection of $\mathrm{A} \beta$ or neuroinflammation by PET [116]. 


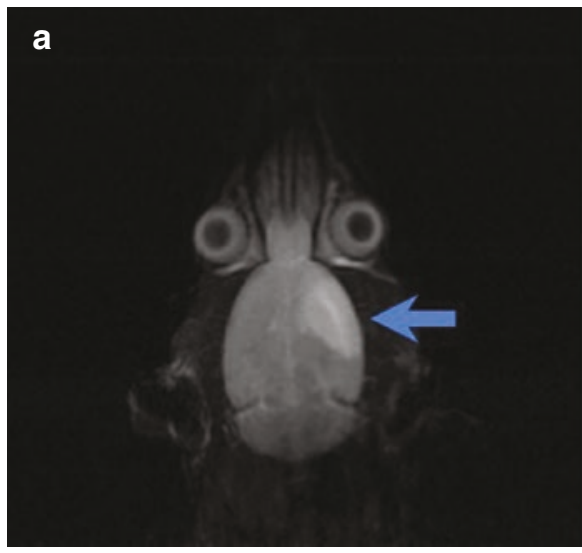

MR (T2WI)

TSPO-PET
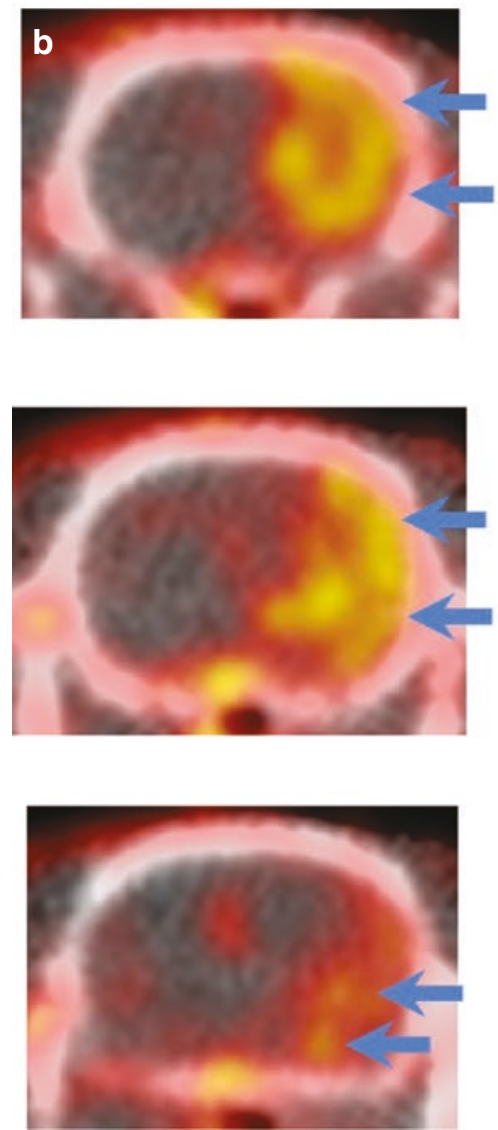

${ }^{11}$ C-DPA713

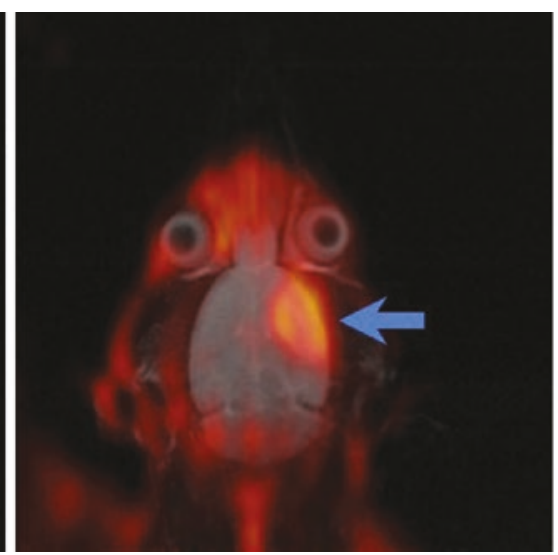

$\mathrm{PET} / \mathrm{MR}$

Fusion

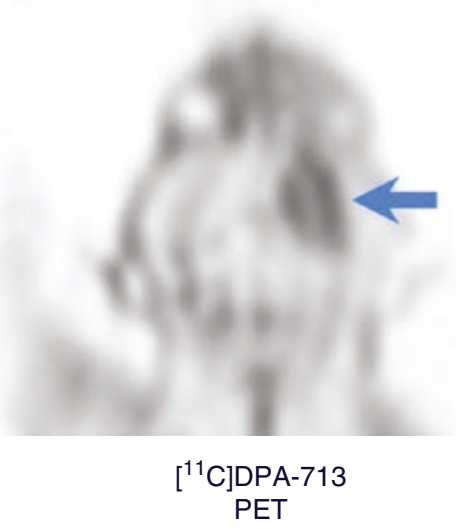

Microglia/Macrophage
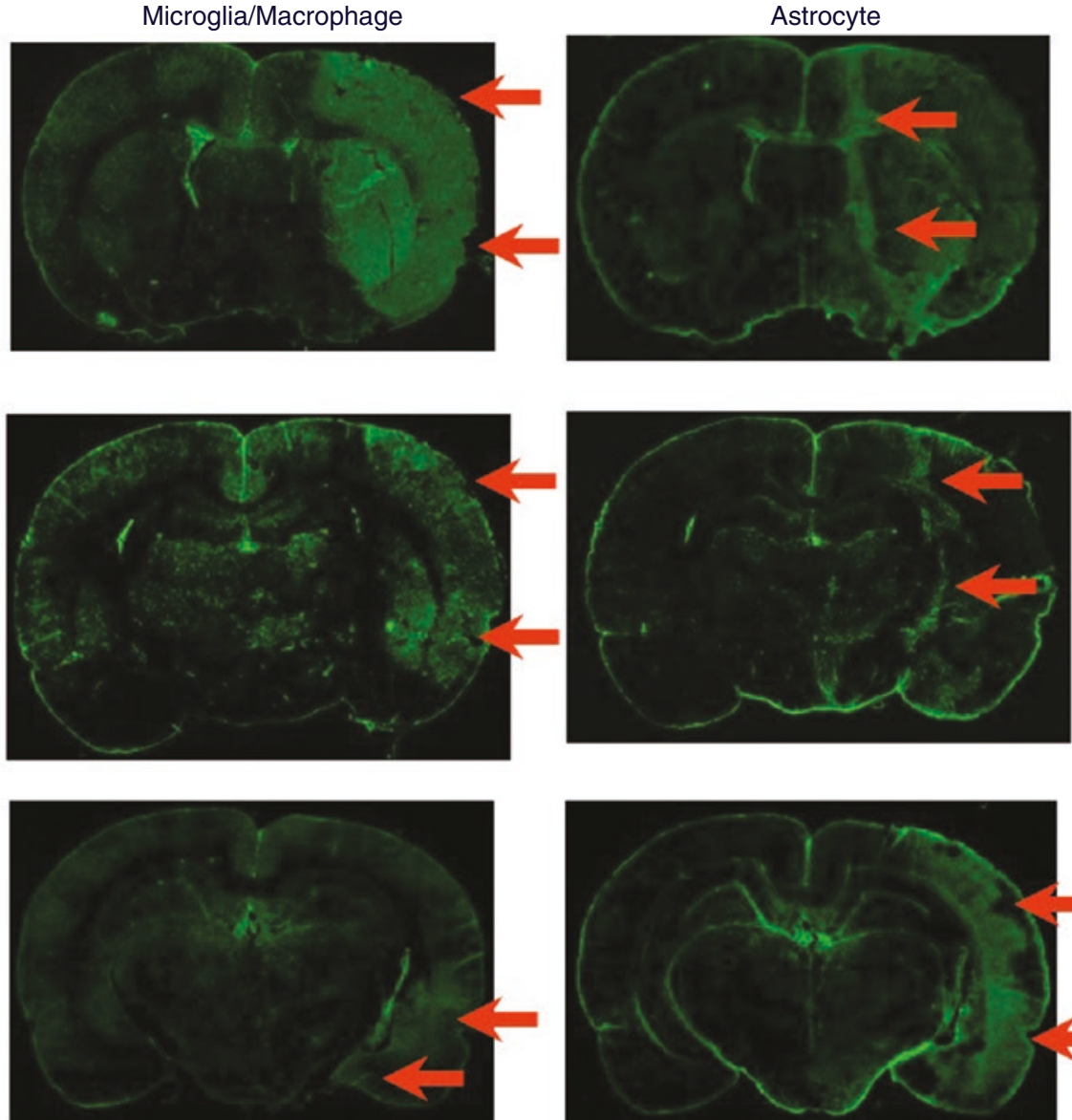

CD11b

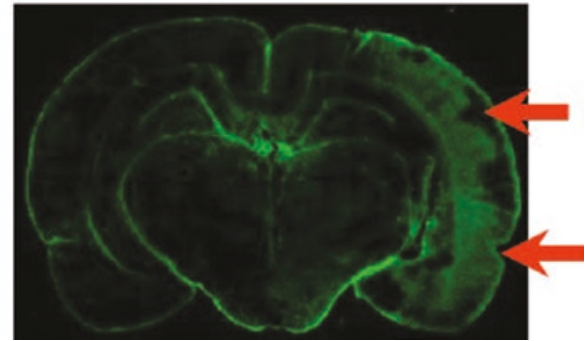

GFAP
Fig. 1.13 (a) TSPO PET imaging of glial activation in the rat brain 4 days after 1-h occlusion of the middle cerebral artery (left: T2-weighted image (T2WI) of MRI, middle: PET/MRI fusion, right: $\left[{ }^{11} \mathrm{C}\right] \mathrm{DPA}-713$ PET). High uptake was observed in the ischemic region corresponding to the hyperintensity lesion visualized on MRI (T2WI) (b) Comparison of TSPO PET and immunofluorescence images. Blue arrows indicate glial activation recognized on $\left[{ }^{11} \mathrm{C}\right] \mathrm{DPA}-713$ PET and red arrows indicate $\mathrm{CD} 11 \mathrm{~b}$ expression in microglia/macrophages or GFAP expression in astrocytes 
Fig. 1.14 $\left[{ }^{18} \mathrm{~F}\right] \mathrm{DPA}-714$

PET/CT images of mice after focal brain injury (static

images obtained 20-30 min after radiotracer

administration). Cortical uptake peaked at 1 week after induction of the injury and shifted to the ipsilateral thalamus after 3 weeks. Arrows indicate glial activation in the ipsilateral side

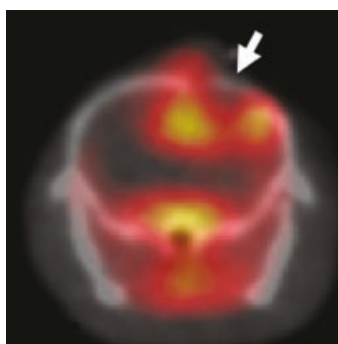

4D

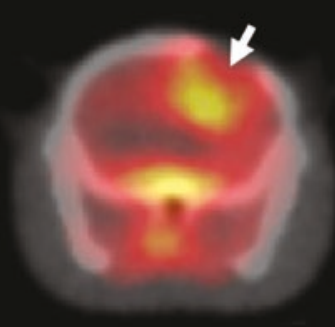

$3 W$

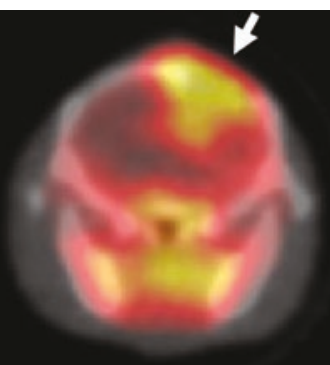

$1 \mathrm{~W}$

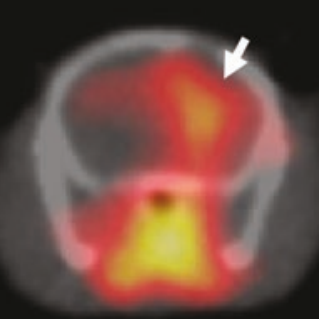

$4 \mathrm{~W}$

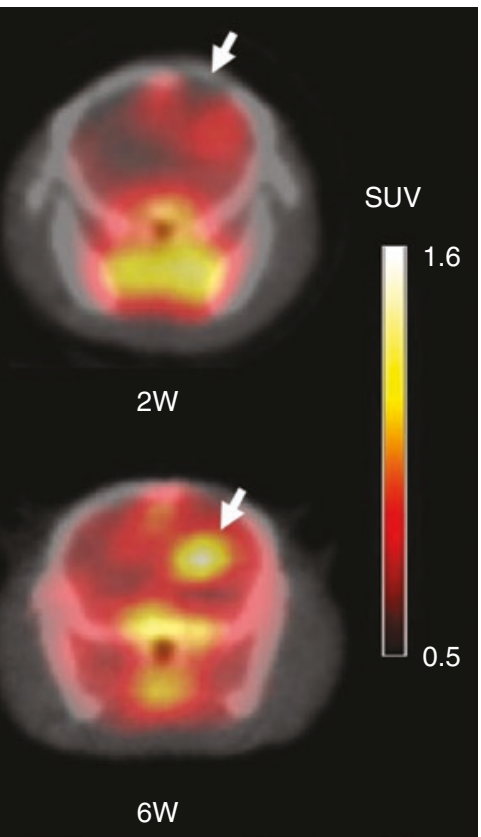

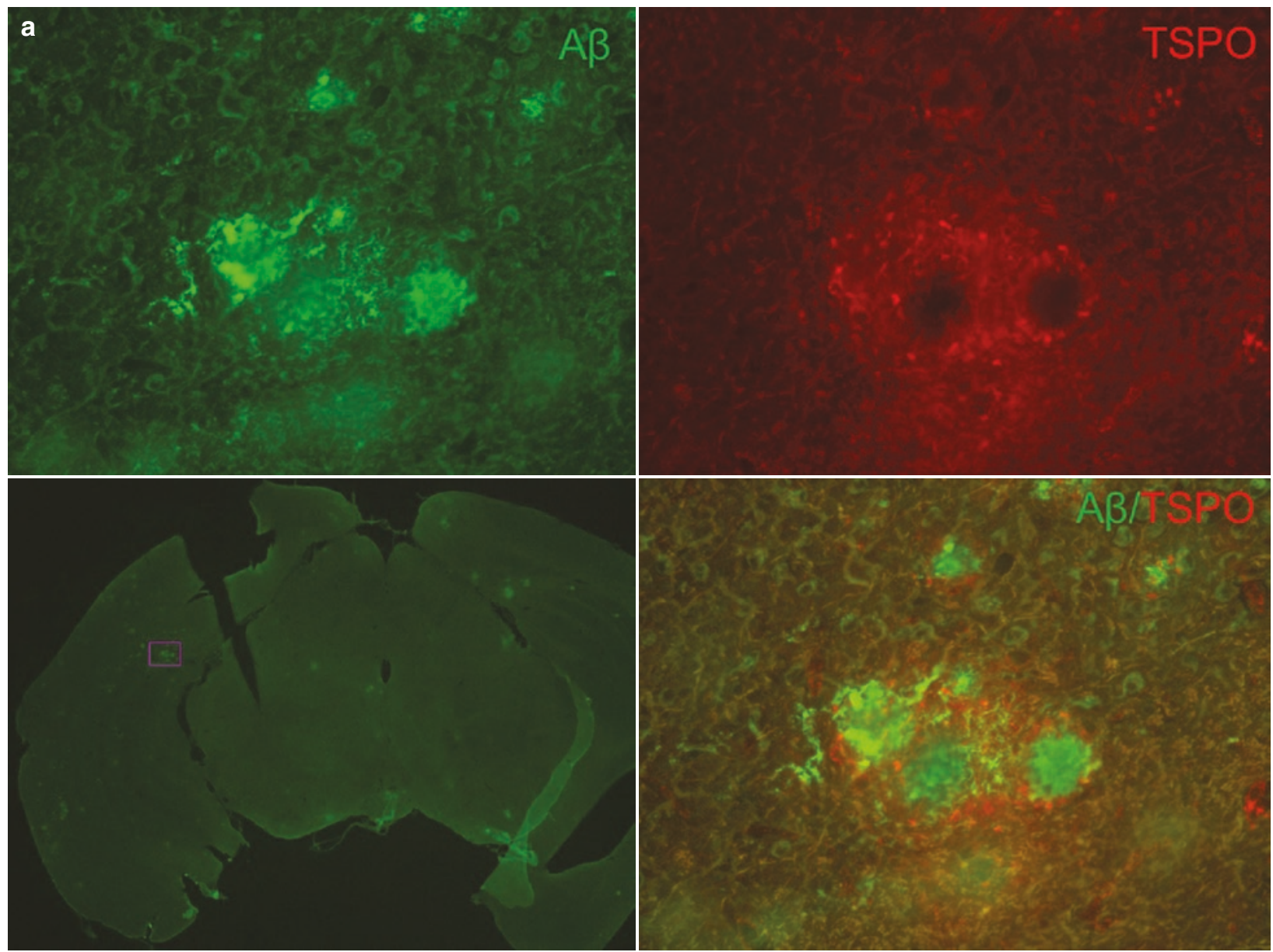

Fig. 1.15 Immunofluorescence images of APP23 Tg mice (15 months old) showing co-staining for (a) A $\beta$ and TSPO, (b) GFAP and TSPO, and (c) CD11b and TSPO. Left lower figure indicates low-magnification images of the brain and the pink-colored square indicates the evaluated area under high magnification. TSPO expression is observed around the amyloid beta $(\mathrm{A} \beta)$ plaques, and GFAP-positive astrocytes show positive co-staining for TSPO. Microglia showing CD11b/TSPO co-staining are relatively few in number as compared to the number of astrocytes showing GFAP/TSPO co-staining. (d) $\left[{ }^{11} \mathrm{C}\right]$ PIB PET images of APP23 Tg mice as compared to wild-type (WT) mice; amyloid deposition is observed in the cortical region and the hippocampus, whereas no significant accumulation is observed in the WT mice 


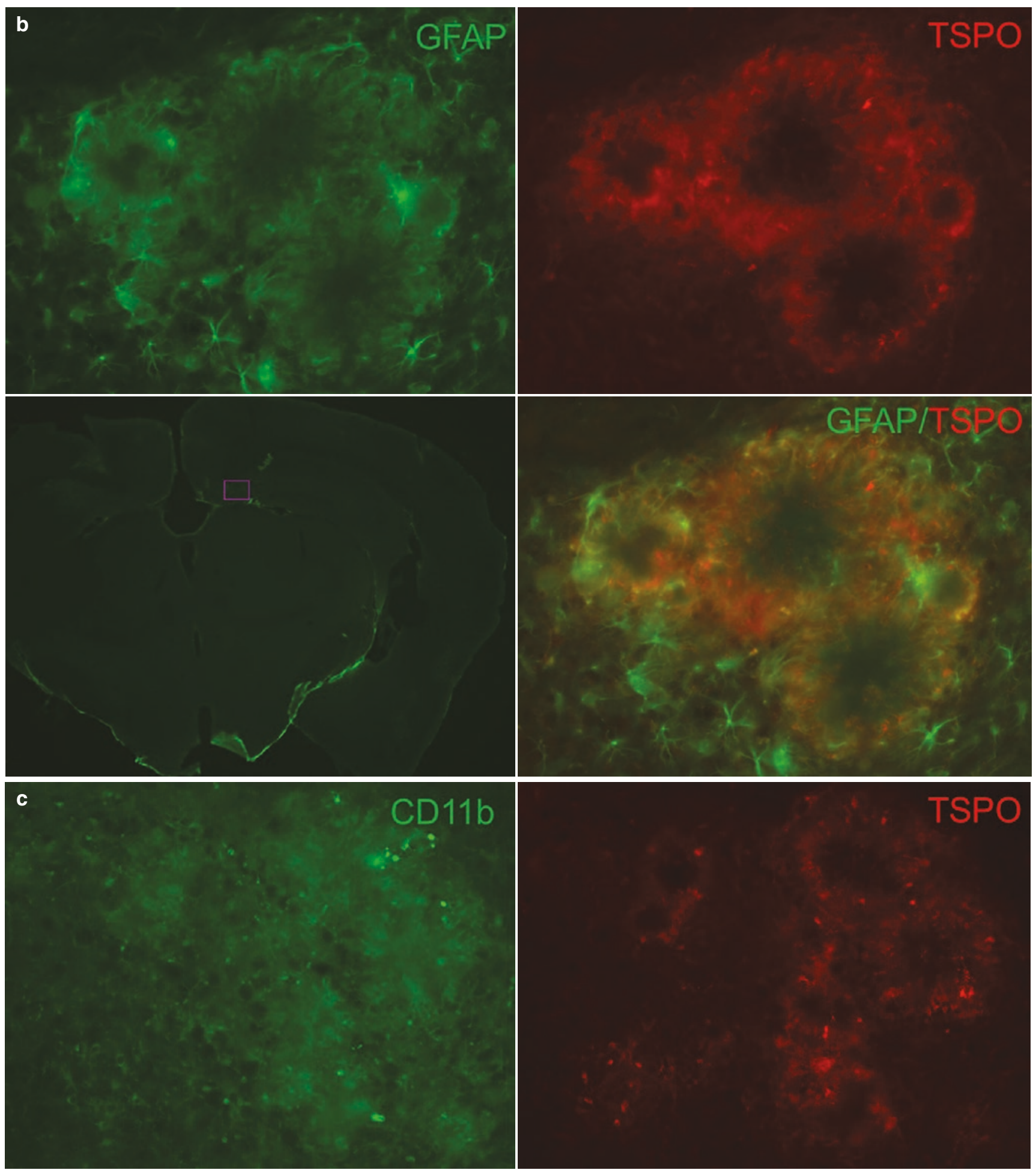

Fig. 1.15 (continued) 


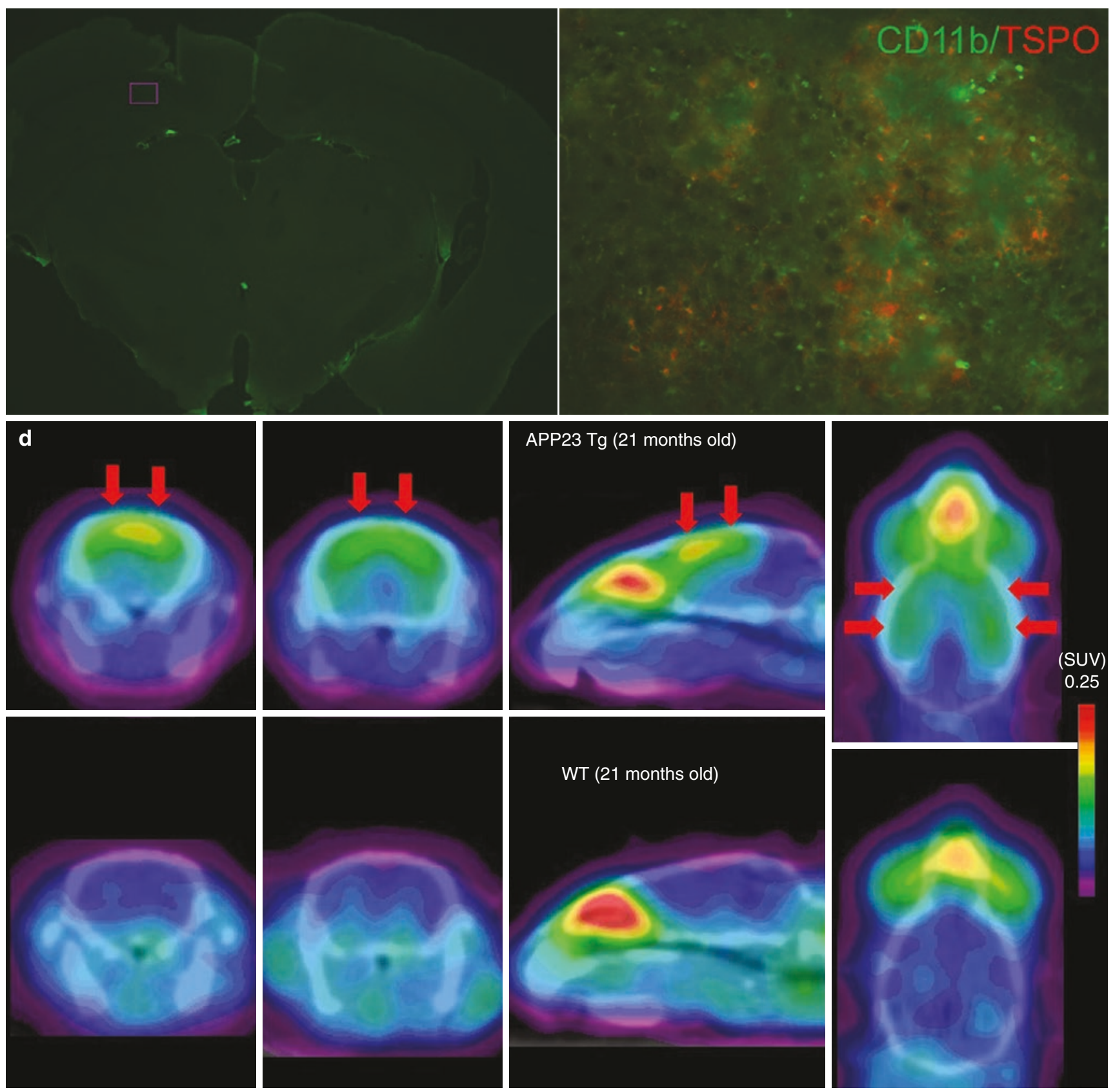

Fig. 1.15 (continued)

Shukuri et al. demonstrated that the expression of cyclooxygenase-1 (COX-1) in activated microglia and macrophages during neuroinflammation can be visualized by PET using $\left[{ }^{11} \mathrm{C}\right]$ ketoprofen methyl ester (KTP-Me), with immunohistochemical confirmation [117]. One advantage of $\left[{ }^{11} \mathrm{C}\right] \mathrm{KTP}-\mathrm{Me}$ PET as compared to TSPO PET is that COX-1 is expressed only in activated microglia and not in astrocytes. They also reported that expression of COX-1 could be detected in 16- to 24-month-old APP23 Tg mice, in accordance with the time of histopathologic appearance of abundant $\mathrm{A} \beta$ plaques and activated microglia [118]. They also reported the possibility of treating $\mathrm{AD}$ by inhibition of COX-1 activity, as a treatment target, with nonsteroidal anti-inflammatory drugs (NSAIDS). Besides TSPO, PET imaging of $\left[{ }^{11} \mathrm{C}\right] \mathrm{KTP}-\mathrm{Me}$ could also be a useful tool for monitoring COX-1 activity in activated microglia in neuroinflammation. 


\subsubsection{Other Models of Neuroinflammation}

Imamoto et al. showed that glial activation could be quantitatively imaged in the spinal cord of a rat model of neuropathic pain using $\left[{ }^{11} \mathrm{C}\right] \mathrm{PK} 11195$ PET [119]. It was suggested that high-resolution PET using TSPO-specific radioligands is useful for imaging to assess the role of glial activation, including neuroinflammatory processes, in the spinal cord. Belolli et al. reported increased enhanced TSPO uptake in a mouse model of multiple sclerosis induced by experimental autoimmune encephalomyelitis (EAE), with neuropathological confirmation of activated microglia [120]. They concluded that combined use of TSPO-PET and MRI could provide complementary evidence of the ongoing disease process.

Evaluation of neuroinflammation by PET in neuropsychiatric disorders, such as schizophrenia and autism spectrum disorder, has been limited to clinical studies, possibly due to the difficulty in establishing appropriate animal models mimicking the pathological condition of the patients.

\subsubsection{New PET Tracer for Neuroinflammation}

As mentioned in Sect. 1.4.4, TSPO-positive glial cells have both neurotoxic and neuroprotective effects. Two phenotypes of microglia have been recognized, namely, neurotoxic "M1" and neuroprotective "M2," although it cannot be clearly separated as M1 or M2 [121]. TSPO-positive microglia that accelerate tau deposition and neuronal deterioration are likely to be M1-polarized. When considering the treatment strategy for neurodegenerative disorders, it is important to detect the detrimental glial cells to suppress their function. Expression of the $\mathrm{P} 2 \mathrm{X} 7$ receptor (purinergic receptor) has been reported to be observed in M1-polarized microglia, which could be a promising target for the detection of deleterious neuroinflammation [122]. In contrast, expression of the P2Y12 receptor has been reported to be observed in the M2-like microglia [123]. Recently, a PET radioligand for the $\mathrm{P} 2 \mathrm{X} 7$ receptor was synthesized and its efficacy was evaluated in preclinical models. Finally, reproducible and dosedependent receptor occupancy studies with a P2X7 receptor antagonist were performed using $\left[{ }^{18} \mathrm{~F}\right] \mathrm{JNJ}-54175446$ in rhesus monkeys and humans $[124,125]$. The usefulness of PET radioligands for the $\mathrm{P} 2 \mathrm{X} 7$ receptor is not only limited to the diagnosis of neuroinflammation. $\left[{ }^{18} \mathrm{~F}\right] \mathrm{PTTP}$, which is also a radioligand targeting the $\mathrm{P} 2 \mathrm{X} 7$ receptor, has been shown to be potentially useful for the quantification of peripheral inflammation targeting macrophages and to distinguish inflammation from certain solid tumors [126]. Other targets in microglia, such as sphingosine-1-phosphate receptor 1 (S1P1) or receptor for advanced glycation end products (RAGE), are also promising, but only in the preliminary stage of investigation at this moment [127].

\subsubsection{Conclusion}

Findings of PET imaging in animal models of neuroinflammation are summarized in this article. In preclinical studies, the pathology can be confirmed by immunohistochemistry or cellular phenotype analysis, as well as by comparison of the PET results. PET imaging targeting TSPO, COX-1, or P2X7 is a promising modality to characterize and monitor neuroinflammation longitudinally and with quantitative accuracy. However, it needs to be borne in mind that preclinical models do not always reflect the pathologies of the heterogeneous characteristics of patients in clinical practice. In addition, we should consider the species differences between rodents and humans. Any findings in preclinical models should be confirmed in clinical situations, which is one of the goals of translational research.

\section{Acknowledgements}

I would like to thank my collaborators: Dr. Sanae Hosomi (Department of Traumatology and Acute Critical Medicine) for the TBI study and Dr. Naoyuki Sato (Department of Clinical Gene Therapy) for the AD study.

\subsection{PET Imaging of Inflammation in the Peripheral System with $\left[{ }^{18} \mathrm{~F}\right]$ FEDAC, a Radioligand for Translocator Protein (18 kDa)}

Ming-Rong Zhang

\begin{abstract}
Translocator protein (18 kDa; TSPO) has a broad array of functions, such as regulation of cholesterol transport, steroid hormone synthesis, porphyrin and heme transport, apoptosis, cell proliferation, anion transport, mitochondrial function regulation, immunomodulation, and inflammation. TSPO is widely expressed in peripheral tissues, including the adrenal gland, kidney, lung, and heart. Because of its pharmacological actions related to inflammation, TSPO has become a useful biomarker for monitoring inflammation using positron emission tomography (PET) with a specific radiotracer. Herein, the author reviews the results of PET imaging with a TSPO-specific radiotracer $\left[{ }^{18} \mathrm{~F}\right] \mathrm{FEDAC}$ to noninvasively visualize and quantify TSPO in the peripheral tissues and monitor various inflammatory diseases, such as lung inflammation, nonalcoholic fatty liver disease, liver damage, liver fibrosis, multiple sclerosis, and rheumatic arthritis, in animal models. $\left[{ }^{18} \mathrm{~F}\right]$ FEDAC-PET is a powerful tool for imaging TSPO and monitoring the progress of various inflammatory diseases of the peripheral system.
\end{abstract}

Keywords: Translocator protein (18 kDa), Lung inflammation, Nonalcoholic fatty liver disease, Liver damage, Liver fibrosis, Multiple sclerosis, Rheumatic arthritis 


\subsubsection{Introduction}

Translocator protein (18 kDa; TSPO) is the critical component of a multimeric $140-200-\mathrm{kDa}$ complex located in the outer mitochondrial membrane and enriched in the outer/inner mitochondrial membrane contact sites [128]. TSPO has broad functions related to the regulation of cholesterol transport, steroid hormone synthesis, porphyrin and heme transport, apoptosis, cell proliferation, anion transport, mitochondrial function regulation, immunomodulation, and inflammation [128]. TSPO is widely distributed in the peripheral tissues, and the mRNA levels of TSPO are high in the adrenal glands, kidneys, spleen, skeletal muscle, heart, and lungs, and are low in the liver and brain. TSPO expression in blood cells has also been reported, in which phagocytic cells, including monocytes and polymorphonuclear neutrophils, show significantly higher TSPO expression than lymphocytes. It has been demonstrated that TSPO expression increases in inflammatory cells during the occurrence and progression of inflammation. Thus, TSPO has become a useful biomarker for monitoring inflammation using PET with a TSPOspecific radiotracer [128].

Since 2002, we have developed more than 10 PET radiotracers for the imaging of TSPO in preclinical studies and have translated four new TSPO radiotracers to clinical studies in our institute: $\left[{ }^{11} \mathrm{C}\right] \mathrm{DAA} 1106 \quad[129-131],\left[{ }^{18} \mathrm{~F}\right]$ FEDAA1106 [132-134], $\left[{ }^{11} \mathrm{C}\right] \mathrm{AC}-5216[135,136]$, and $\left[{ }^{18} \mathrm{~F}\right]$ FEDAC [137-139]. Here, the author reviews their results from PET with $\left[{ }^{18} \mathrm{~F}\right] \mathrm{FEDAC}$ to noninvasively visualize TSPO in the peripheral tissues [140] and monitor various inflammatory diseases, such as lung inflammation [141], nonalcoholic fatty liver disease [142], liver damage [143], liver fibrosis [144], multiple sclerosis [145], and rheumatic arthritis [146, 147], in animal models.

\subsubsection{PET Imaging and Quantitative Analysis of TSPO in Rat Peripheral Tissues}

Using small-animal PET with $\left[{ }^{18} \mathrm{~F}\right] \mathrm{FEDAC}$, we performed TSPO imaging to quantify TSPO density in rat peripheral tissues, including heart, lungs, and kidneys [140]. The in vivo distribution and kinetics of $\left[{ }^{18} \mathrm{~F}\right]$ FEDAC were first examined and measured in rat peripheral tissues. Using the in vivo pseudo-equilibrium method, TSPO binding parameters (TSPO density $\left[B_{\max }\right]$ and dissociation constant $\left[K_{\mathrm{D}}\right]$ ) and receptor occupancy were estimated in the peripheral tissues.

PET study showed that the uptake of radioactivity was highly distributed in the lungs, heart, and kidneys, and the TSPO-enriched tissues could be clearly visualized after the injection of $\left[{ }^{18} \mathrm{~F}\right]$ FEDAC (Fig. 1.16). The kinetics of this radiotracer in the tissues was moderate, which is suitable for determining the in vivo binding parameters and receptor occupancy. The $B_{\max }$ values of TSPO in the heart, lung, and kidney were 393,141 , and $158 \mathrm{pmol} / \mathrm{mL}$, respectively (Table 1.1). The $K_{\mathrm{D}}$ values of $\left[{ }^{18} \mathrm{~F}\right] \mathrm{FEDAC}$ in the heart, lung, and kidney were 119,36 , and $123 \mathrm{nM}$, respectively. After pretreatment with $5 \mathrm{mg} / \mathrm{kg}$ Ro 5-4864 (a TSPO-selective ligand), about $90 \%$ of binding sites for TSPO in the heart and lung were occupied. In the kidneys, the TSPO binding was also completely inhibited by Ro 5-4864.

Through this study, we demonstrated that $\left[{ }^{18} \mathrm{~F}\right]$ FEDAC is a useful PET tracer for TSPO imaging and quantitative analysis of TSPO expression in peripheral tissues. Therefore, $\left[{ }^{18}\right.$ F]FEDAC-PET can be used to study the TSPO function and evaluate the in vivo binding parameters and receptor occupancy of TSPO ligands. Subsequently, we used PET with $\left[{ }^{18} \mathrm{~F}\right] \mathrm{FEDAC}$ to determine if TSPO increases in inflamed peripheral tissues of various animal models.
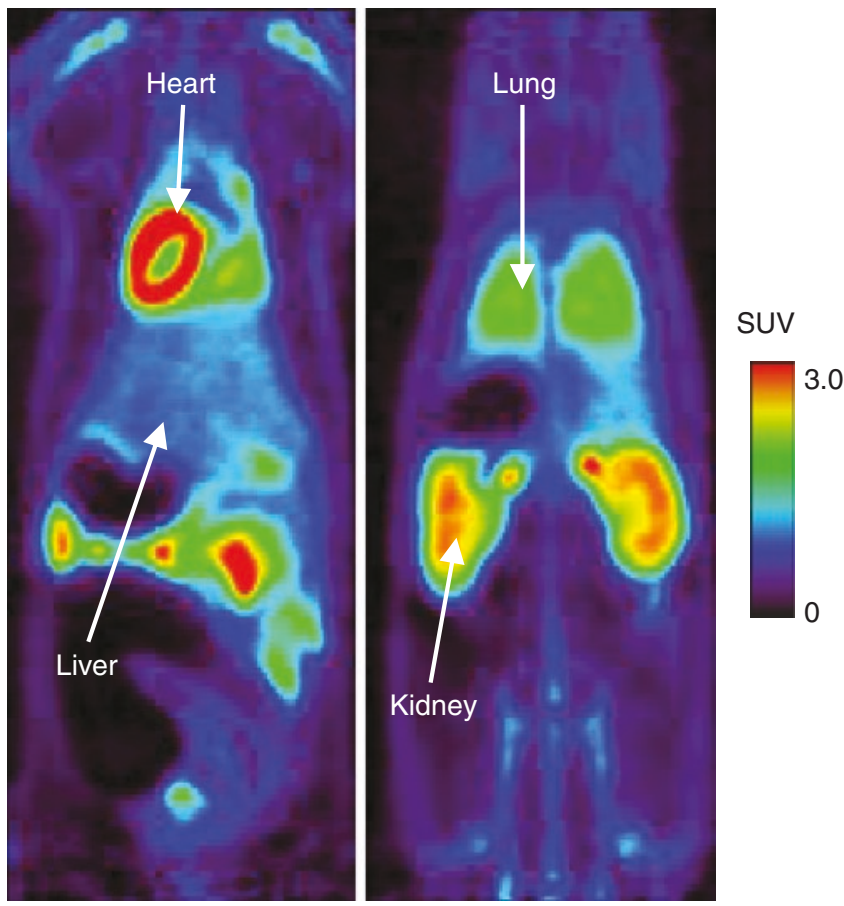

Fig. 1.16 Representative coronal PET images of $\left[{ }^{18} \mathrm{~F}\right]$ FEDAC in the isoflurane-anesthetized rat, which was placed in the prone position. PET images were generated by summating the whole scan ( $0-30 \mathrm{~min})$

Table 1.1 TSPO density $\left(B_{\max }\right)$ and dissociation constant $\left(K_{\mathrm{D}}\right)$ of $\left[{ }^{18} \mathrm{~F}\right]$ FEDAC measured by PET in peripheral tissues of living rats

\begin{tabular}{l|l|l}
\hline Tissue & $B_{\max }(\mathrm{pmol} / \mathrm{mL})$ & $K_{\mathrm{D}}(\mathrm{nM})$ \\
\hline Heart & $393[323-529]^{\mathrm{a}}$ & $119[89-180]^{\mathrm{a}}$ \\
\hline Lung & $141[112-205]^{\mathrm{a}}$ & $119[26-61]^{\mathrm{a}}$ \\
\hline Kidney & $158[129-217]^{\mathrm{a}}$ & $123[92-191]^{\mathrm{a}}$ \\
\hline
\end{tabular}

a95\% Confidence intervals $(n=3)$ 


\subsubsection{TSPO in Lung Inflammation}

TSPO is highly expressed on the bronchial and bronchiole epithelium, submucosal glands in intrapulmonary bronchi, pneumocytes and alveolar macrophages in human lungs. We performed PET imaging of lung inflammation with $\left[{ }^{18} \mathrm{~F}\right]$ FEDAC and determined cellular sources that enrich TSPO in the lung [141]. We prepared an acute lung injury model by using intratracheal administration of lipopolysaccharide (LPS) to rats. PET with $\left[{ }^{18} \mathrm{~F}\right] \mathrm{FEDAC}$ demonstrated that, in response to LPS treatment, the uptake of radioactivity increased in the lungs as the inflammation progressed (Fig. 1.17). Pretreatment with a TSPO-selective unlabeled ligand PK11195 significantly decreased the lung uptake of $\left[{ }^{18} \mathrm{~F}\right]$ FEDAC because of competitive binding to TSPO. TSPO expression was elevated in the inflamed lung section and its level responded to the $\left[{ }^{18} \mathrm{~F}\right] \mathrm{FEDAC}$ uptake and severity of inflammation. The presence of TSPO was examined in the lung tissue using western blotting and immunohistochemical assays. The increase of TSPO expression was mainly found in the neutrophils and macrophages of inflamed lungs (Fig. 1.18).

Through this study, we demonstrated that PET with $\left[{ }^{18} \mathrm{~F}\right]$ FEDAC is a useful tool for imaging TSPO expression and evaluating the progression of lung inflammation.

\subsubsection{TSPO in Nonalcoholic Fatty Liver Disease}

Mitochondrial dysfunction is responsible for liver damage and disease progression in nonalcoholic fatty liver disease (NAFLD). TSPO, as a mitochondrial transmembrane protein, plays important roles in regulating mitochondrial function. We conducted PET with $\left[{ }^{18} \mathrm{~F}\right] \mathrm{FEDAC}$ to explore if TSPO could be used as an imaging biomarker of noninvasive diagnosis and staging of NAFLD [142]. PET with $\left[{ }^{18} \mathrm{~F}\right]$ FEDAC, CT, autoradiography, histopathology, and gene analysis were performed to evaluate and quantify TSPO levels and NAFLD progression in methionine and cholinedeficient diet-fed mice. The uptake of $\left[{ }^{18} \mathrm{~F}\right] \mathrm{FEDAC}$ increased with disease progression from simple steatosis to nonalcoholic steatohepatitis (NASH) (Fig. 1.19). A strong correlation was observed between $\left[{ }^{18} \mathrm{~F}\right]$ FEDAC uptake ratio and NAFLD activity score in the liver. The specific binding of $\left[{ }^{18} \mathrm{~F}\right]$ FEDAC to TSPO in the NAFLD livers was demonstrated by competition experiments with the unlabeled TSPO-selective PK11195. Autoradiography and histopathology supported these PET imaging results. Further, there were greater mRNA levels of the functional macromolecular signaling complex composed of TSPO, compared to controls (Fig. 1.20).
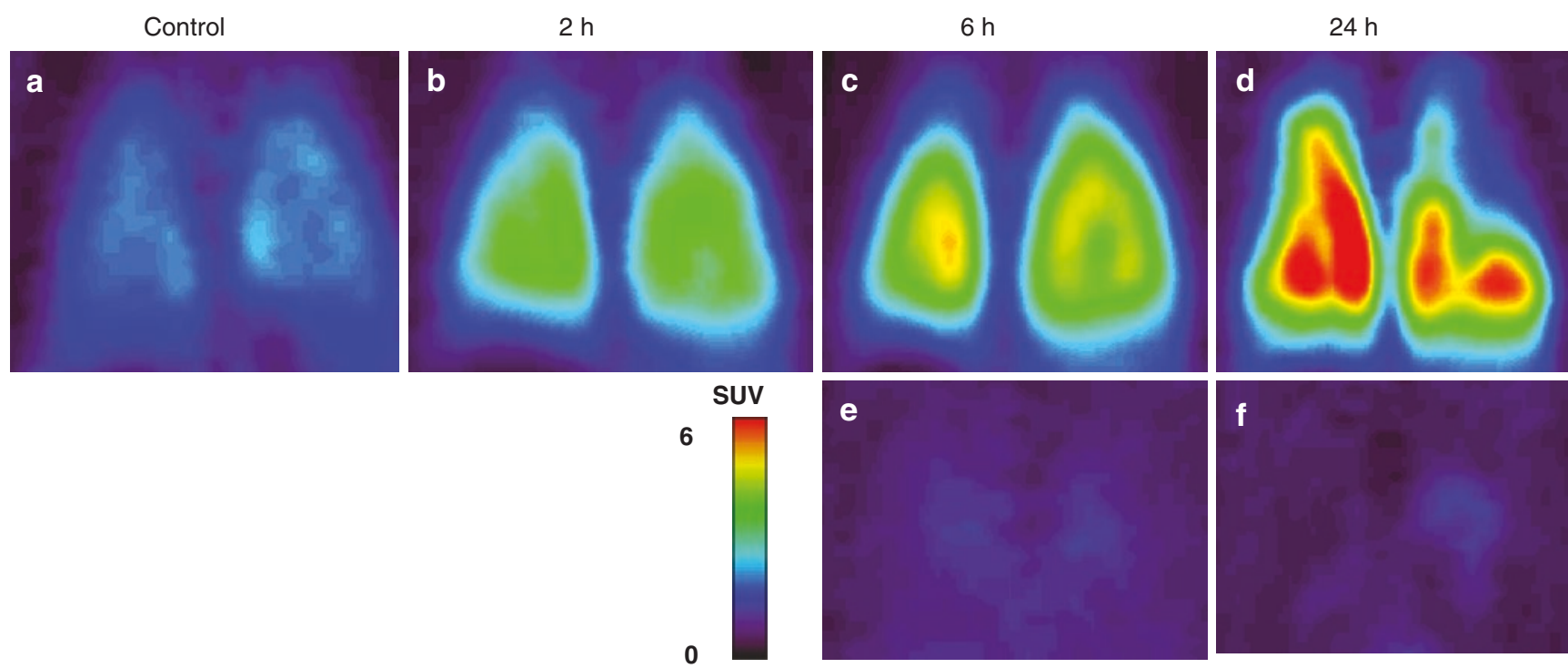

PK11195 treatment

Fig. 1.17 Representative coronal PET lung images acquired between 0 and 30 min after injection of $\left[{ }^{18}\right.$ F]FEDAC. (a) control; (b) LPS-2 h; (c) LPS-6 h; (d) LPS-24 h inducement; pretreatment with (e) PK11195 for LPS-6 h; (f) PK11195 for LPS-24 h 


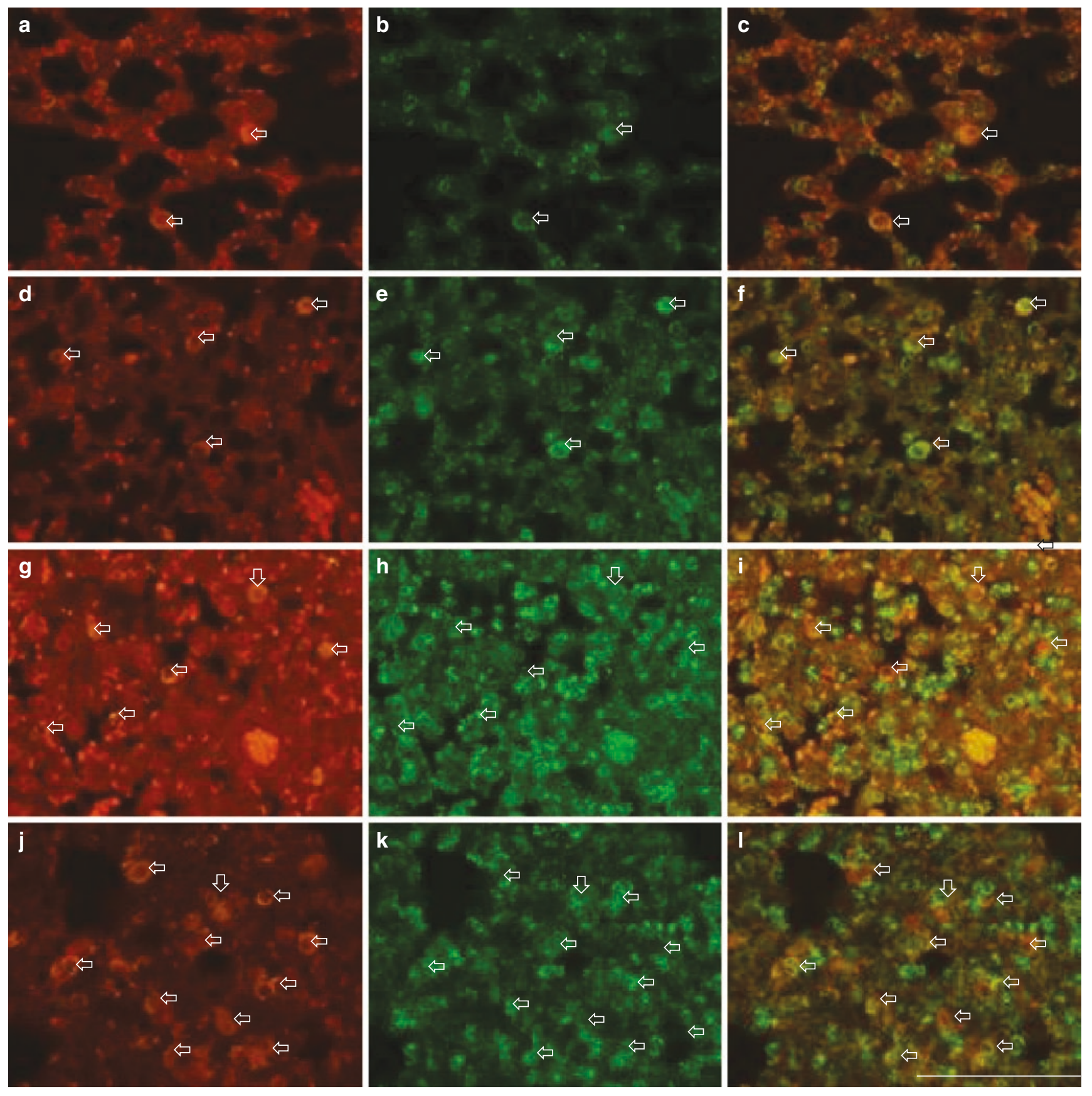

Fig. 1.18 Double immunofluorescence labeling of ED1 (red) and TSPO (green) displayed as a two-channel image. The images demonstrated temporal alterations of TSPO expression in macrophages at $2 \mathrm{~h}$ $(\mathbf{d}-\mathbf{f}), 6 \mathrm{~h}(\mathbf{g}-\mathbf{i})$, and $24 \mathrm{~h}(\mathbf{j}-\mathbf{l})$ after LPS inducement, and in the control $(\mathbf{a}-\mathbf{c})$. Arrows indicate examples of cells doubly positive for ED1 and

Through this study, we demonstrated that TSPO expression increased in NAFLD and was strongly correlated with NAFLD progression. TSPO as a specific molecular imaging biomarker could provide a novel tool for noninvasive, reliable, and quantitative diagnosis and staging of NAFLD.
TSPO. (a-c) TSPO expression was observed in a few macrophages. (d-f) Several macrophages demonstrating TSPO immunoreactivity were observed in the alveolar spaces. $(\mathbf{g}-\mathbf{i}, \mathbf{j}-\mathbf{l})$ Numbers of macrophages with TSPO gradually increased over time. Scale bar: $20 \mu \mathrm{m}$

\subsubsection{TSPO in Liver Damage}

Liver damage induced by drug toxicity is an important problem for both medical doctors and patients. In this study, we noninvasively visualized acute liver damage using PET with $\left[{ }^{18} \mathrm{~F}\right] \mathrm{FEDAC}$ and determined cellular 

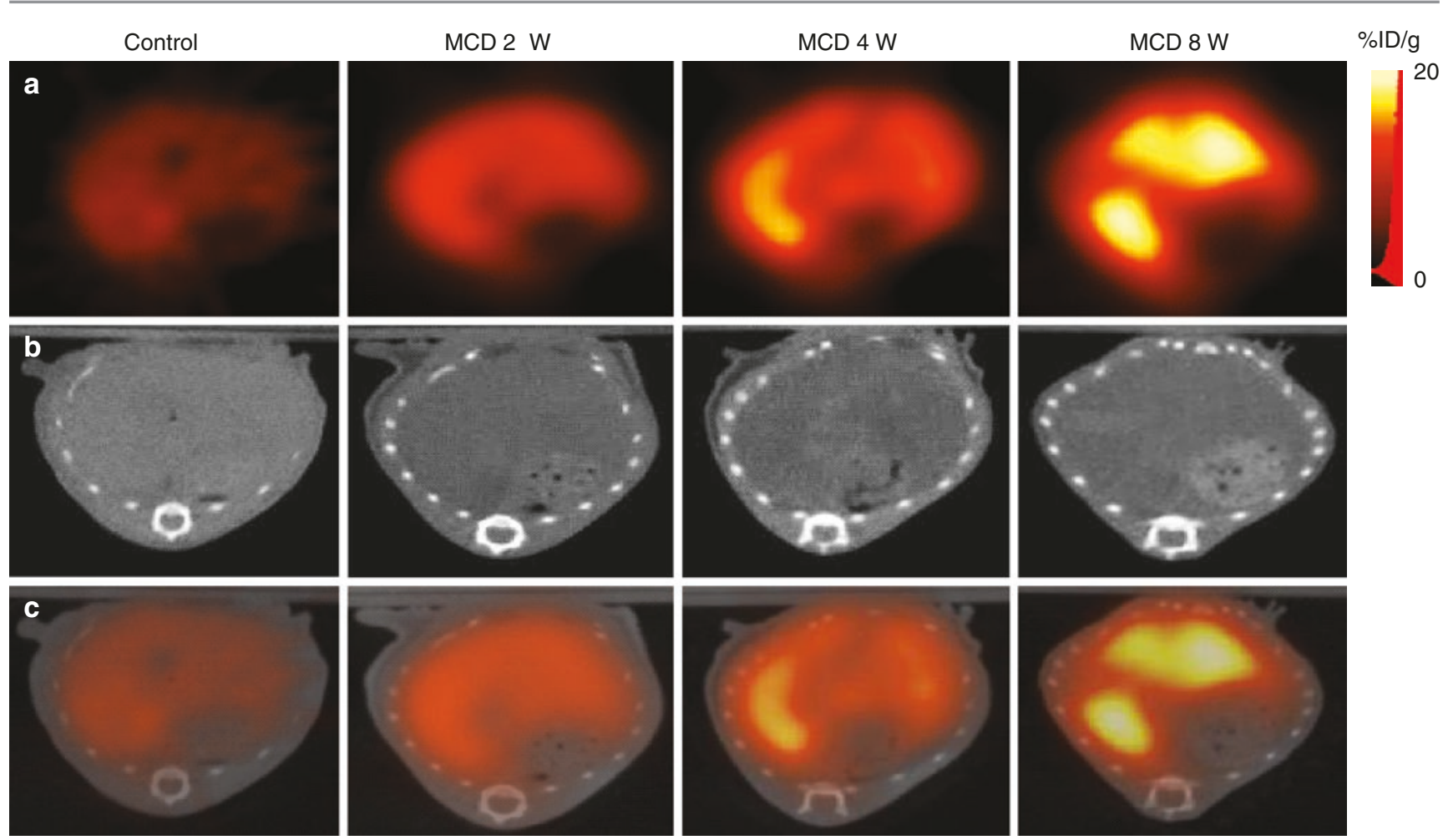

Fig. 1.19 Representative TSPO-PET/CT images of livers in MCD and control mice. (a) Summed TSPO-PET images of 0-30 min scanning in the $2,4,8$ weeks MCD-fed mice and the 8 weeks normal-fed mice. $\left[{ }^{18} \mathrm{~F}\right]$ FEDAC-PET signal gradually increased compared to the controls. (b)

Average unenhanced CT images. Decreased attenuation value in the parenchyma was presented in all stages of NALFD. (c) TSPO-PET/CT fusion images. More severe pathologic injures took place in the areas of higher radioactivity accumulation

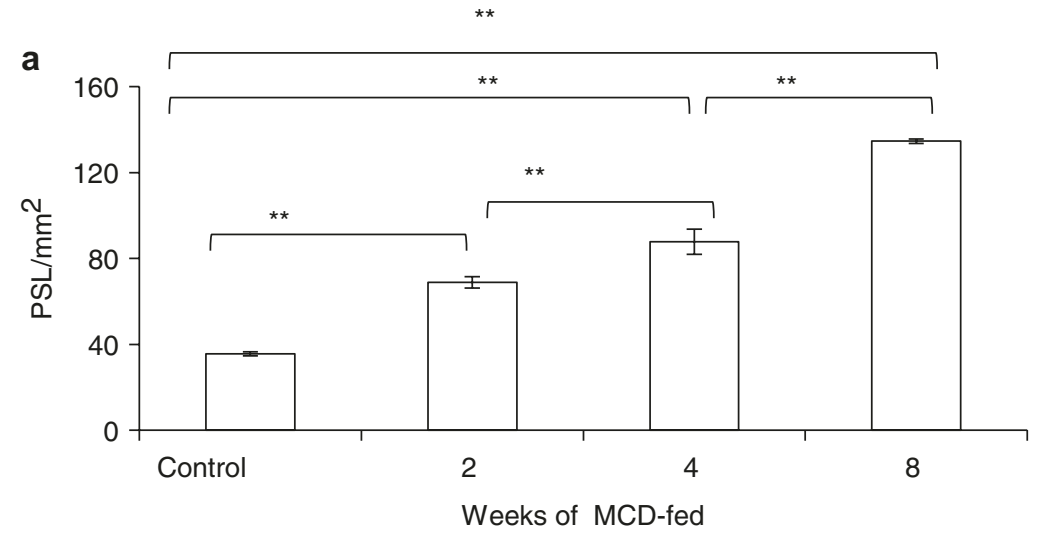

Fig. 1.20 Ex vivo autoradiography and immunohistochemistry in liver sections of MCD and control mice ( $n=6$, respectively). (a) Ex vivo autoradiography demonstrated that radioactivity gradually increased in the livers with the time of MCD feeding. (b) Representing autoradiographic images of liver sections. (c) Double immunohistochemistry including TSPO (blue) and CD11b (orange). Preferential localization of TSPO indicated in the liver lesion sites, CD11b/TSPO active macrophages and lymphocytes increased in the MCD livers (Scale bars $=500 \mu \mathrm{m}) . * * P<0.01$ 


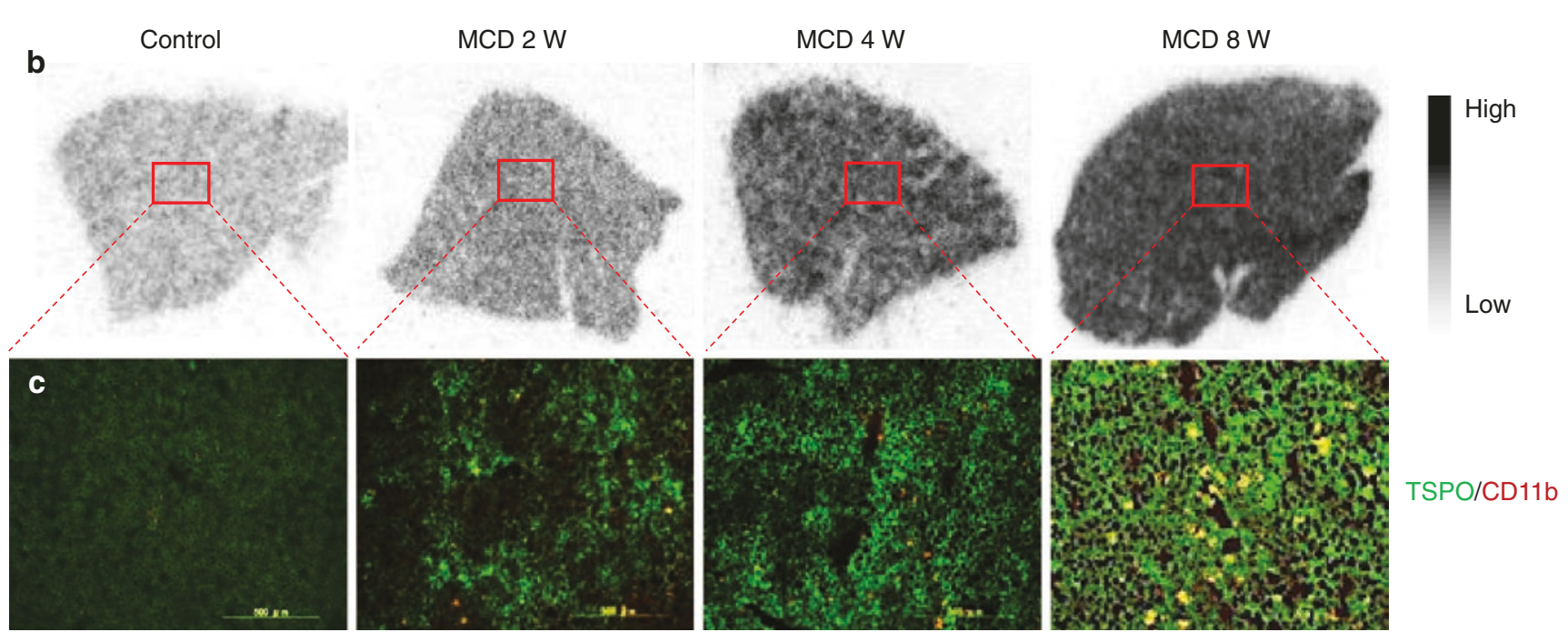

Fig. 1.20 (continued)

Control

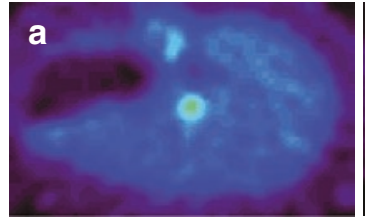

b

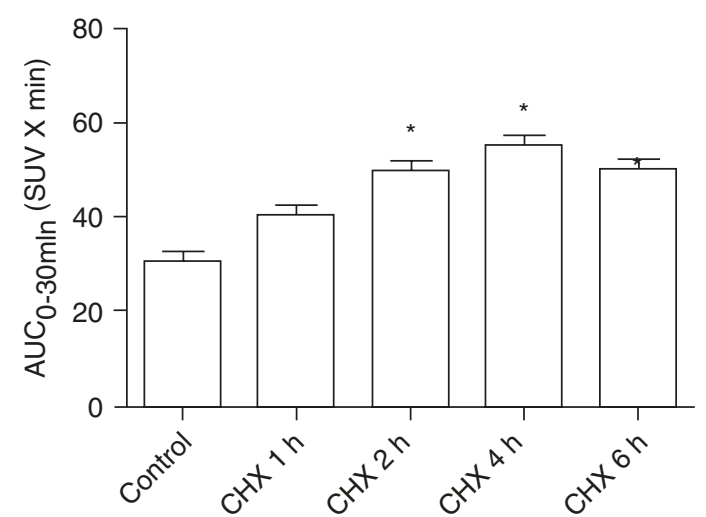

Fig. 1.21 Radioactivity and TSPO level in the livers of the control and CHX-treated rats. (a) Representative transverse PET liver images acquired between 0 and $30 \mathrm{~min}$ after injection of $\left[{ }^{18} \mathrm{~F}\right] \mathrm{FEDAC}$. (b) Values of areas under time-activity curves. The values $\left(\mathrm{AUC}_{0-30 \mathrm{~min}}\right.$; $\mathrm{SUV} \times$ min, mean $\pm \mathrm{SEM}, n=4)$ were calculated from the time-activity

sources enriching TSPO expression in the liver [143]. A mild acute liver damage model was prepared by a single intraperitoneal injection of cycloheximide (CHX) to rats. Treatment with CHX induced apoptosis and necrotic changes in hepatocytes with a slight neutrophil infiltration. The uptake of radioactivity in the rats' livers was measured with PET after injection of $\left[{ }^{18} \mathrm{~F}\right]$ FEDAC. PET with $\left[{ }^{18} \mathrm{~F}\right]$ FEDAC showed that the uptake of radioactivity increased in livers treated with $\mathrm{CHX}$, compared to the controls curves between 0 and $30 \mathrm{~min}$ after the injection. A significant difference $(P<0.05)$ was observed on the following comparisons, $*$ : control vs. CHX $2 \mathrm{~h}, 4 \mathrm{~h}$, and $6 \mathrm{~h}$. (c) Effect of CHX treatment on the mRNA expression of TSPO. A significant difference $(P<0.05)$ was observed on the following comparisons, $*$ : control vs. CHX treatment
(Fig. 1.21). The mRNA expression of TSPO was increased in the damaged livers compared to the controls, and the TSPO level was correlated with the $\left[{ }^{18} \mathrm{~F}\right] \mathrm{FEDAC}$ uptake and severity of damage. TSPO expression in the damaged liver sections was mainly found in macrophages (Kupffer cells) and neutrophils, but not in hepatocytes (Fig. 1.22). The increase in expression of TSPO mRNA was induced by an increase in the numbers of macrophages and neutrophils with TSPO in the damaged livers. 


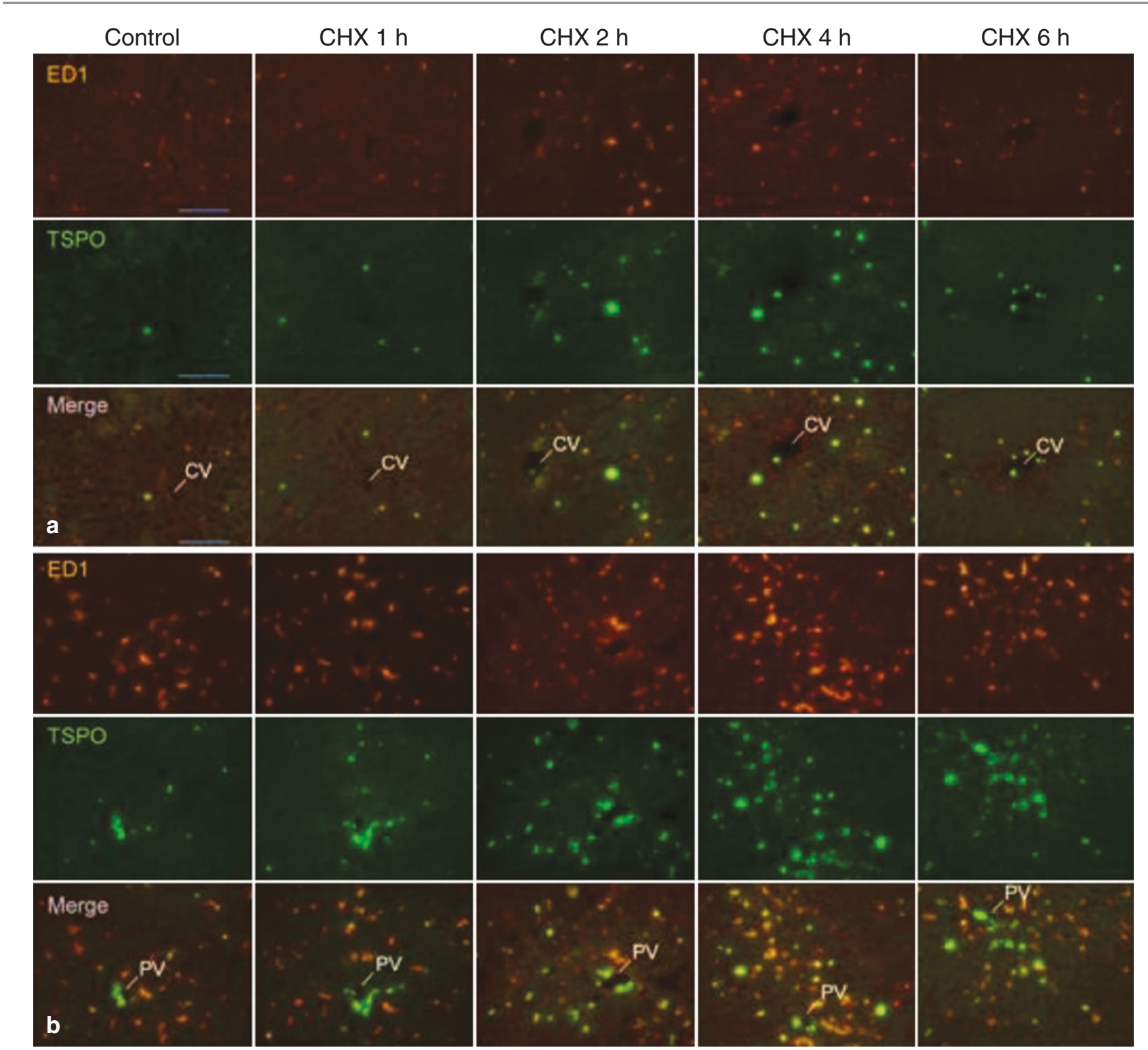

Fig. 1.22 Increased TSPO levels were expressed in macrophages in liver tissues after CHX treatment. Double immunofluorescence labeling of $\mathrm{ED} 1$ (red) and TSPO (green) in the centrilobular region (a) and the periportal region (b). $C V$ central vein, $P V$ portal vein. Scale bar: $100 \mu \mathrm{m}$

Through this study, we demonstrated that PET imaging with $\left[{ }^{18} \mathrm{~F}\right]$ FEDAC provided visible evidence that mild liver damage occurs through the enhanced TSPO signal in inflammatory cells, and that this method is a useful diagnostic tool in the early stages of acute liver damage.

\subsubsection{TSPO in Liver Fibrosis}

Liver fibrosis is the wound healing response to chronic liver injury, which is caused by various factors. In this study, we evaluated the utility of TSPO as a molecular imaging biomarker for monitoring the progression of liver fibrosis in response to cirrhosis [144]. To induce cirrhosis, carbon tetrachloride $\left(\mathrm{CCl}_{4}\right)$ was administered to rats and the subsequent liver fibrosis was assessed. PET with $\left[{ }^{18} \mathrm{~F}\right] \mathrm{FEDAC}$ was used to noninvasively visualize liver fibrosis in vivo. PET scanning, immunohistochemical staining, ex vivo autoradiography, and quantitative reverse-transcription polymerase chain reaction were performed to elucidate the relationships among radioactivity uptake, TSPO level, and cellular source enriching TSPO expression in damaged livers. PET with $\left[{ }^{18} \mathrm{~F}\right] \mathrm{FEDAC}$ showed that the uptake of radioactivity in livers significantly increased after 2, 4, 6, and 8 weeks of $\mathrm{CCl}_{4}$ treatment (Fig. 1.23). Immunohistochemical analysis demonstrated that TSPO was primarily expressed in macrophages and liver stellate cells (HSCs). TSPO-expressing macrophages and HSCs increased with the progression of liver fibrosis (Fig. 1.24). The distribution of radioactivity from $\left[{ }^{18} \mathrm{~F}\right] \mathrm{FEDAC}$ was strongly correlated with TSPO expression, and TSPO mRNA levels increased with the severity of liver damage. 


\section{Period for $\mathrm{CCl}_{4}$ injection}
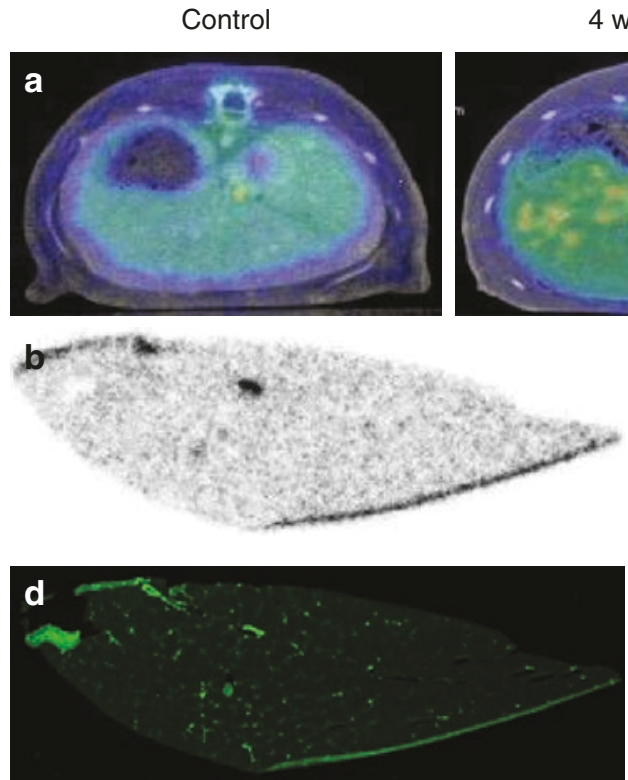

Fig. 1.23 Accumulation of radioactivity and TSPO level in the livers of control and $\mathrm{CCl}_{4}$-treated rats. (a) Representative transverse PET/CT fusion images of rat livers. PET images were acquired between 0 and 30 min after injection of $\left[{ }^{18} \mathrm{~F}\right]$ FEDAC. (b) Autoradiographic image of liver section $1 \mathrm{~h}$ after injection of $\left[{ }^{18} \mathrm{~F}\right] \mathrm{FEDAC}$ is shown for control rat. (c) Autoradiographic image is shown for rat treated with $\mathrm{CCl}_{4}$ for
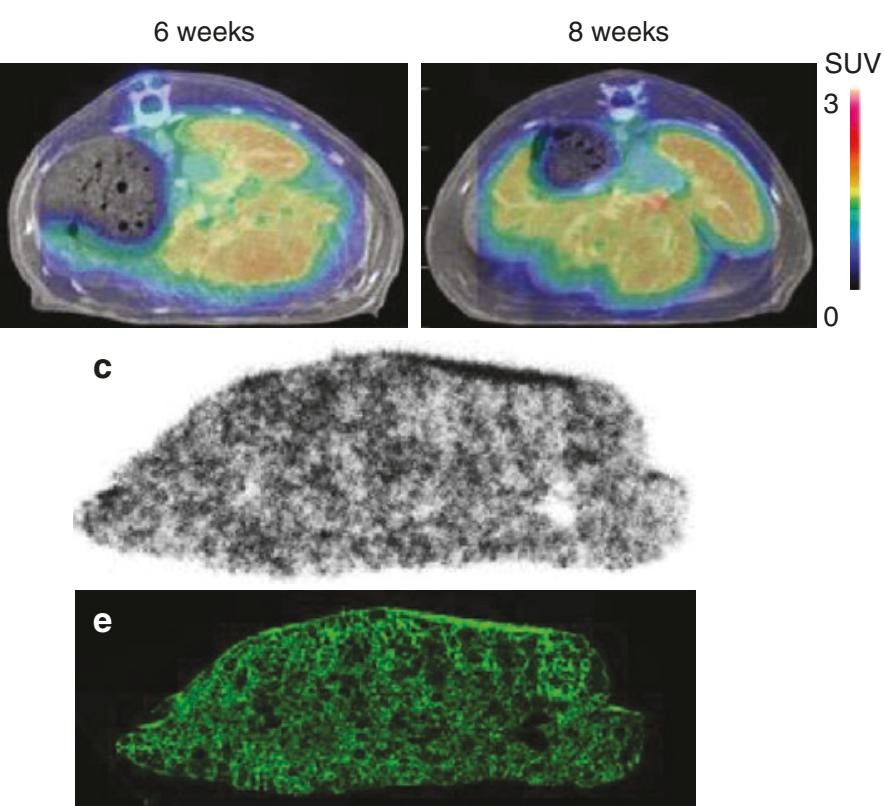

6 weeks. (d) Immunofluorescence staining of TSPO using the same sections as in (a) is shown for control rat. (e) Immunofluorescence staining and rats treated with $\mathrm{CCl}_{4}$ for 6 weeks. The distribution of radioactivity from $\left[{ }^{18} \mathrm{~F}\right] \mathrm{FEDAC}$ correlated well with the netlike appearance of TSPO expression in the hepatic lobules (c, e). Scale bar: $1 \mathrm{~mm}$

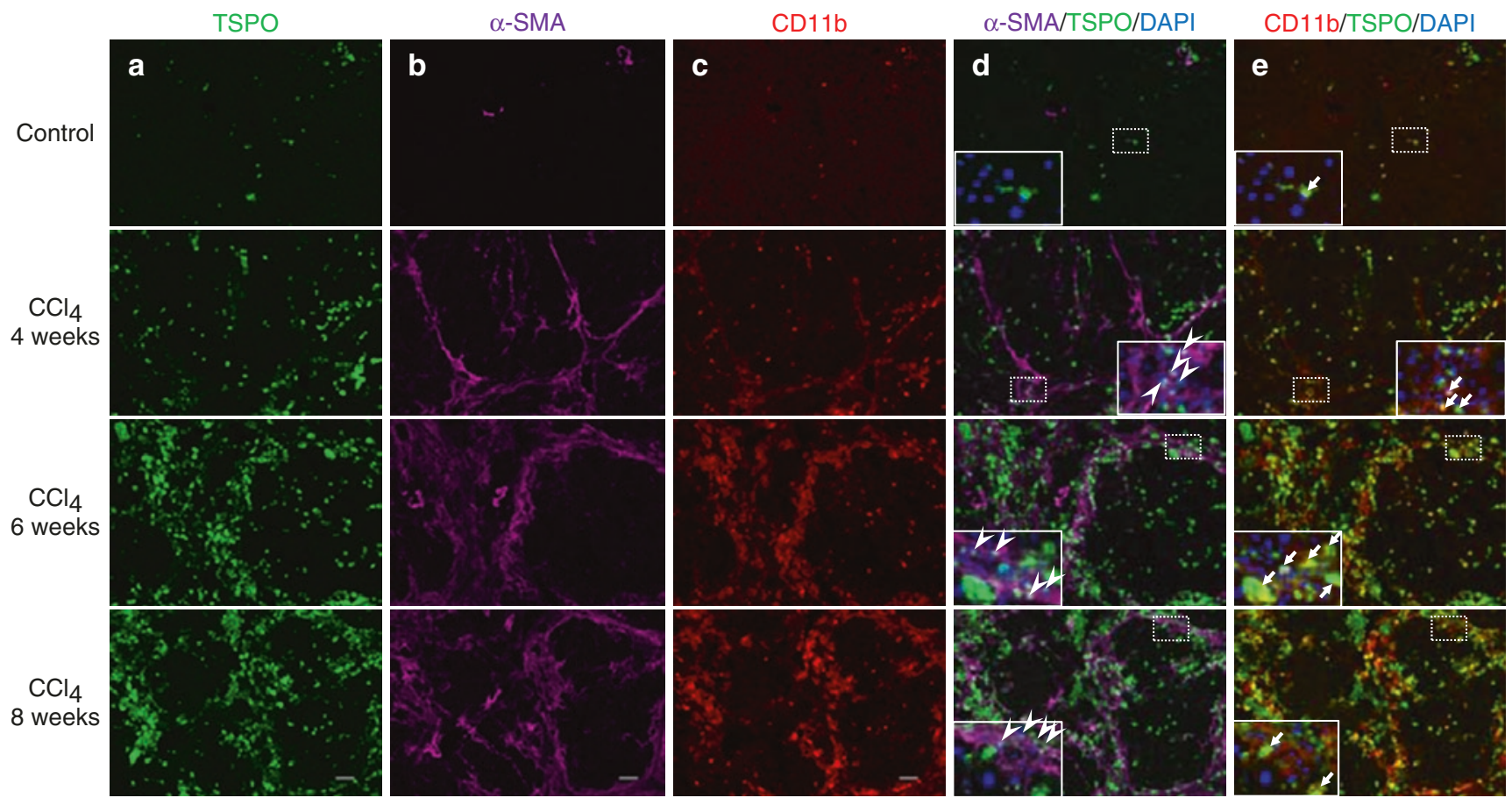

Fig. 1.24 Increased levels of TSPO expressed in HSCs and macrophages from liver tissues after $\mathrm{CCl}_{4}$ treatment. Triple immunofluorescence labeling of TSPO (a, green), $\alpha$-SMA (b, pink), CD11b (c, red), and DAPI (blue) in liver sections from control animals and animals treated with $\mathrm{CCl}_{4}$ for 4,6 , or 8 weeks $(\mathbf{a}-\mathbf{e})$. (d and e) Show merged images of (a) and (b) and of (a) and (c), respectively. The dotted line square in each slide $(\mathbf{d}, \mathbf{e})$ is enlarged and shown in the inset with DAPI. Arrowheads point to TSPO double-stained HSCs (a-SMApositive cells). Arrows point to TSPO double-stained macrophages (CD11b-positive cells). Scale bar: $50 \mu \mathrm{m}$ 
Through this study, we demonstrated that TSPO is a useful molecular imaging biomarker for monitoring the progression of liver fibrosis in response to cirrhosis with PET.

\subsubsection{TSPO in Multiple Sclerosis}

Imaging modalities have the potential to monitor inflammation in order to guide treatment before significant functional impairment or irreversible cellular damage occurs in multiple sclerosis (MS). In this study, $\left[{ }^{11} \mathrm{C}\right] \mathrm{DAC}$, a carbon-11labeled TSPO radiotracer derived from $\left[{ }^{18} \mathrm{~F}\right] \mathrm{FEDAC}$, was used to evaluate neuroinflammation and quantify the therapeutic effects of experimental autoimmune encephalomyelitis (EAE), an animal model of MS [145]. PET with $\left[{ }^{11} \mathrm{C}\right]$ DAC was used to visually assess neuroinflammation in EAE by measuring TSPO expression in the spinal cords; there was the maximal uptake on day 11 and 20 EAE rats with significant inflammatory cell infiltration, compared to controls, day 0 and 60 EAE rats (Fig. 1.25). Biodistribution studies and in vitro autoradiography confirmed the PET imaging results. Doubling immunohistochemical studies demonstrated the infiltration and expansion of CD4+ T cells and CD11b+ microglia; CD68+ macrophages caused the increase in TSPO levels visualized by $\left[{ }^{11} \mathrm{C}\right] \mathrm{DAC}-\mathrm{PET}$. Furthermore, mRNA level analysis of the cytokines using quantitative reversetranscription polymerase chain reaction revealed that TSPO+/CD4 T cells, TSPO+ microglia, and TSPO+ macrophages in EAE spinal cords were activated and secreted multiple pro-inflammation cytokines, which mediated the inflammation lesions of EAE. The EAE rats treated with an immunosuppressive agent FTY720 exhibited an absence of inflammatory cell infiltrates and displayed a faint radioactive signal, compared to the high accumulation in untreated EAE rats (Fig. 1.26).

Through this study, we demonstrated that $\left[{ }^{11} \mathrm{C}\right] \mathrm{DAC}-\mathrm{PET}$ imaging is a useful tool for noninvasively monitoring the neuroinflammation response and evaluating therapeutic interventions in EAE.

\subsubsection{TSPO in Rheumatic Arthritis}

Rheumatoid arthritis (RA) is a chronic disease characterized by systemic inflammation that results in the destruction of multiple articular cartilages and bones. Activated macrophages have been known to play important roles in the pathogenesis of rheumatoid arthritis (RA). Cheon et al. evaluated the feasibility of $\left[{ }^{18} \mathrm{~F}\right] \mathrm{FEDAC}-\mathrm{PET}$ in a murine RA model [146]. In the collagen-induced arthritis (CIA) mice, joint swelling was apparent on day 26 after the first immunization, and the condition worsened by day 37. In these mice, the mRNA and protein expression of the TSPO increased in activated macrophages. The uptake of $\left[{ }^{18} \mathrm{~F}\right] \mathrm{FEDAC}$ in activated macrophages was greater compared to the uptake in nonactivated cells, which was inhibited by PK11195. The $\left[{ }^{18} \mathrm{~F}\right]$ FEDAC uptake by arthritic joints increased early on (day 23), whereas $\left[{ }^{18} \mathrm{~F}\right]$ FDG uptake did not (Fig. 1.27). However, $\left[{ }^{18} \mathrm{~F}\right] \mathrm{FDG}$ uptake by arthritic joints markedly increased at later stages (day 37) to a higher level than $\left[{ }^{18} \mathrm{~F}\right]$ FEDAC uptake. The $\left[{ }^{18} \mathrm{~F}\right]$ FEDAC uptake correlated weakly with the summed severity score, whereas the $\left[{ }^{18} \mathrm{~F}\right] \mathrm{FDG}$ uptake correlated strongly with the summed severity score. Histologic sections of arthritic joints demonstrated an influx of macrophages compared to that in normal joints (Fig. 1.28). Moreover, these indicated that PET imaging using $\left[{ }^{18} \mathrm{~F}\right]$ FEDAC may be used as a predictor of the therapeutic effects caused by biological disease-modifying antirheumatic drugs that have anti-inflammatory actions to inhibit activated macrophages [147].

Through these results, it was demonstrated that $\left[{ }^{18} \mathrm{~F}\right]$ FEDAC enabled the visualization of active inflammation sites in arthritic joints in a CIA model by targeting TSPO
Fig. 1.25 Representative PET images acquired by summed whole scan duration of 0-30 min after $\left[{ }^{11} \mathrm{C}\right] \mathrm{DAC}$ injection. $\left[{ }^{11} \mathrm{C}\right] \mathrm{DAC}-\mathrm{PET}$ signal increased significantly in day 7,11 , and $20 \mathrm{EAE}$ spinal cords

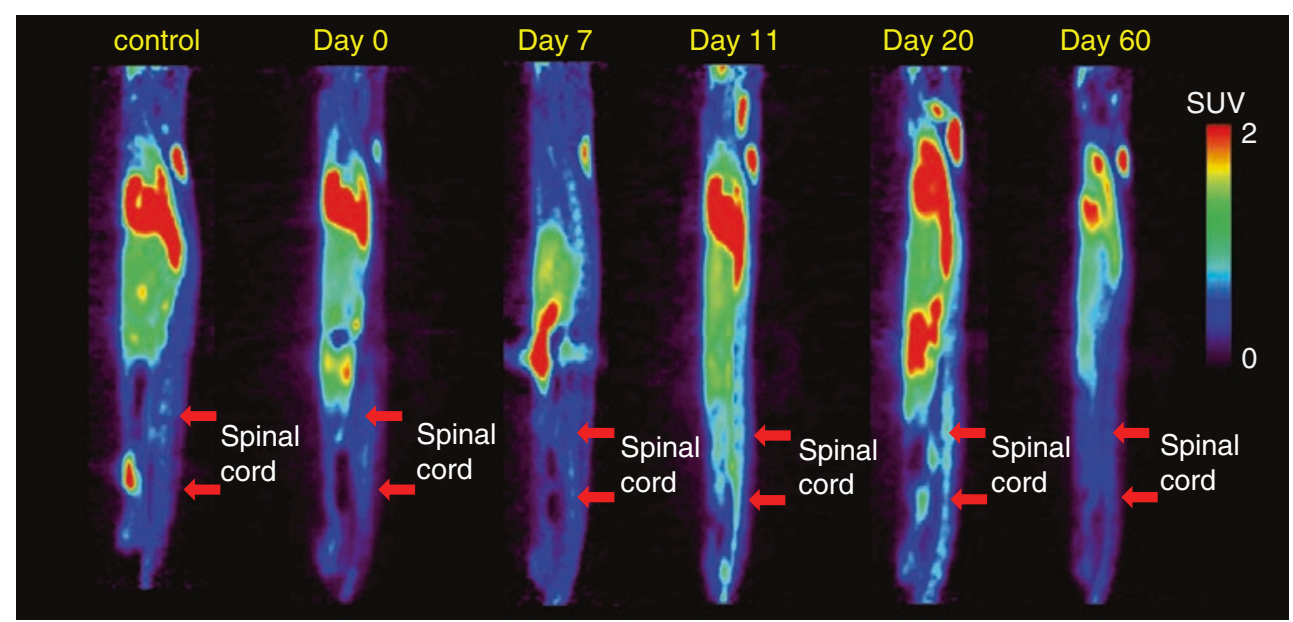


Fig. 1.26 Reduced uptake of $\left[{ }^{11} \mathrm{C}\right] \mathrm{DAC}$ in EAE by FTY720 treatment. (a) $\left[{ }^{11} \mathrm{C}\right] \mathrm{DAC}$-PET scans were performed at 11 days EAE rats that treated or untreated with FTY720 ( $n=8$, respectively). Uptake of $\left[{ }^{11} \mathrm{C}\right] \mathrm{DAC}$ was clearly increased only in day 11 untreated rats, uptake in FTY720-treated rats remained low and unchanged, similar to naïve rats. Reduced uptake of inhibition of neuroinflammation in EAE by FTY720 treatment. (b-d) $\mathrm{H} \& \mathrm{E}$ staining of day 11 spinal sections indicated that rats with FTY720 treatment exhibited a complete absence of inflammatory cell infiltrates compared to the compressive inflammatory lesions in untreated rats $\left[{ }^{11} \mathrm{C}\right] \mathrm{DAC}$ reflected the
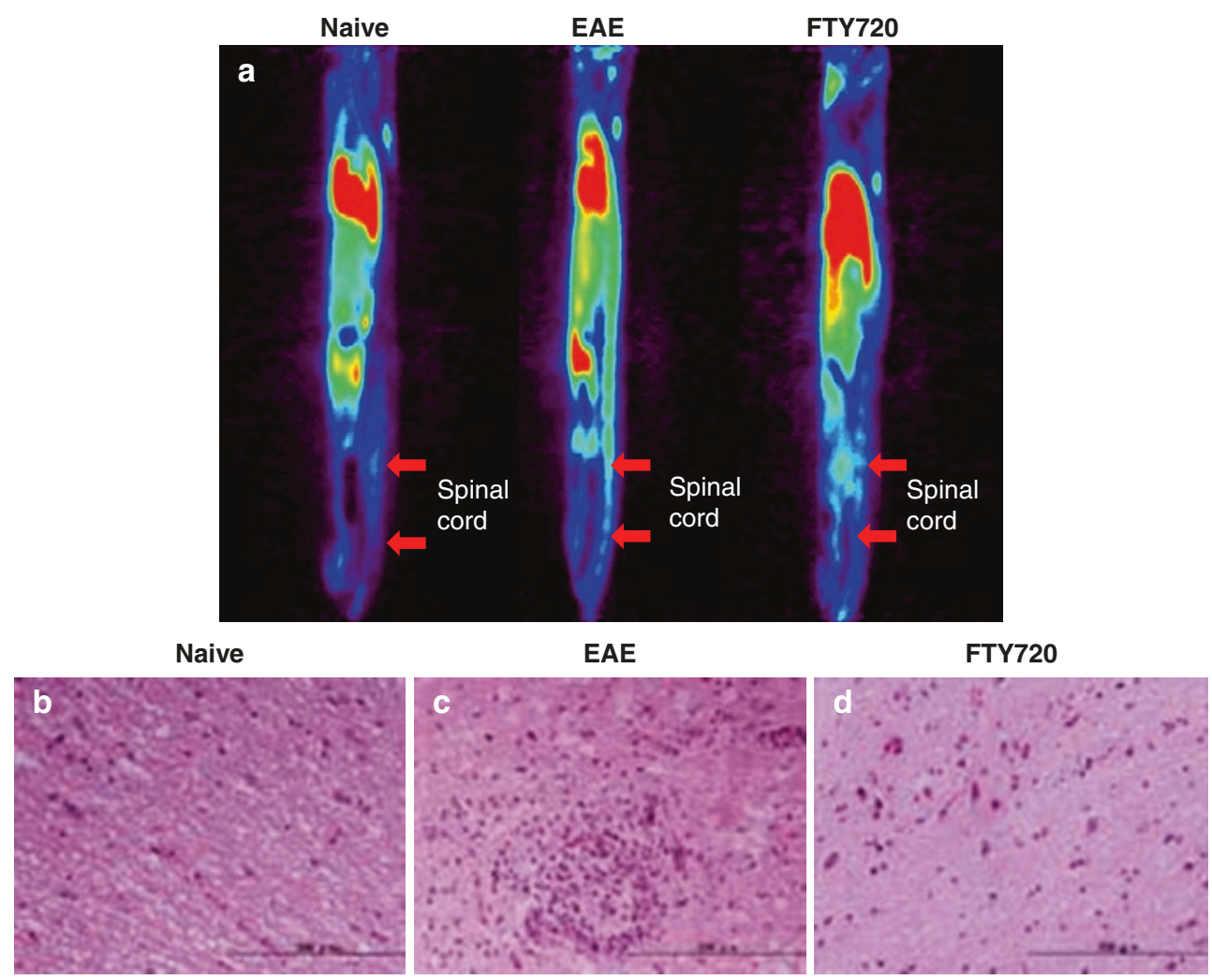

$\left[{ }^{18}\right.$ F $]$ FEDAC
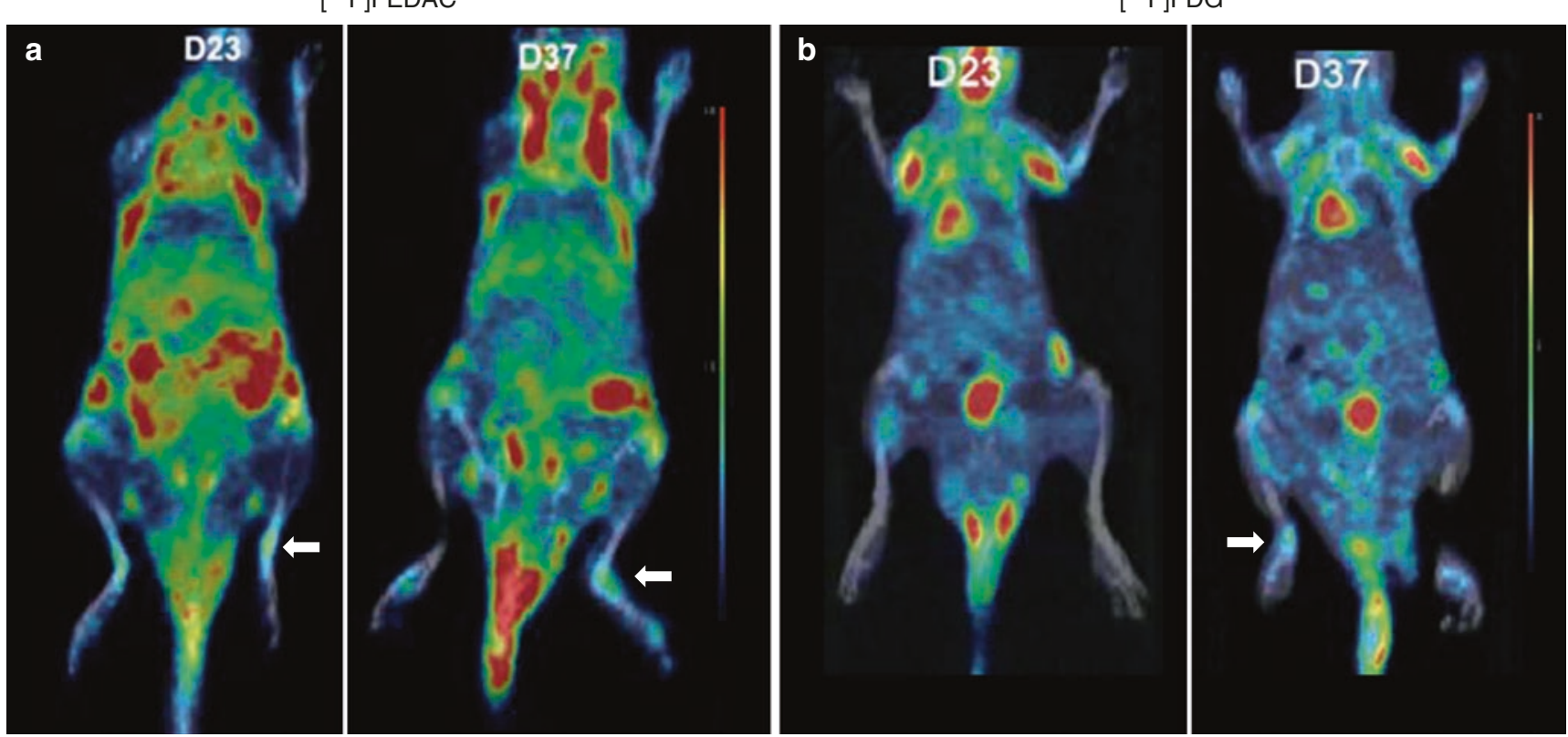

Fig. 1.27 (a) Coronal $\left[{ }^{18} \mathrm{~F}\right]$ FEDAC PET/CT section of same CIA mouse on days 23 and 37, showing increased uptake in front and hind paws. (b) Coronal section of $\left[{ }^{18} \mathrm{~F}\right] \mathrm{FDG}$ PET/CT $(\mathbf{a}, \mathbf{b})$ in the same mouse. On day 23, uptake by joints was not observed before the onset of arthritis. On day 37 , increased $\left[{ }^{18} \mathrm{~F}\right] \mathrm{FDG}$ uptake was observed for all four arthritic joints 

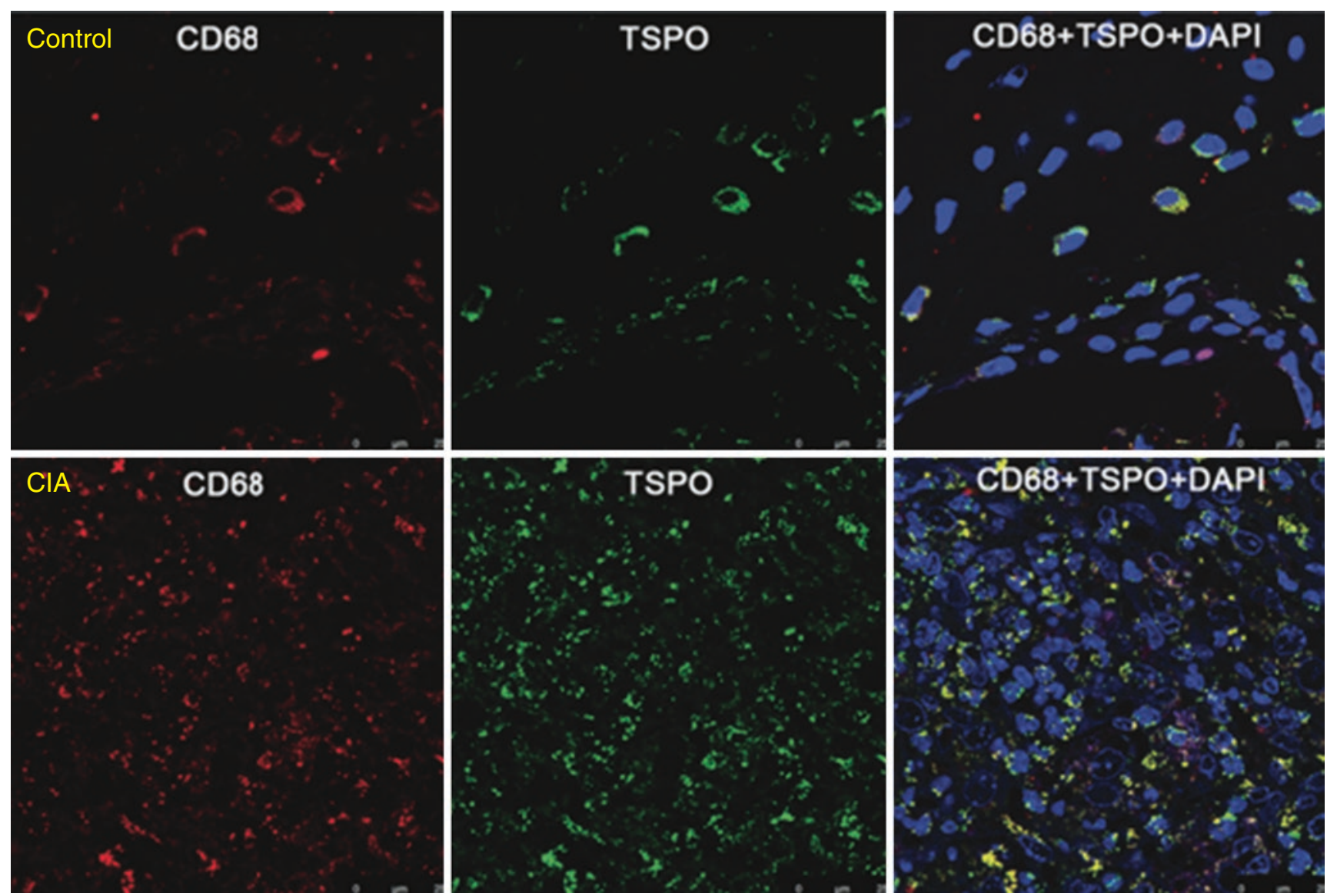

Fig. 1.28 Immunofluorescence staining for CD68 (red) and TSPO (green) in synovium of control and CIA joints. Merged images at far right show increased number and TSPO expression of CD68-positive macrophages, compared to controls

expression in activated macrophages. The results suggest the potential usefulness of $\left[{ }^{18} \mathrm{~F}\right]$ FEDAC-PET imaging in the early phase of RA.

\subsubsection{Conclusion}

$\left[{ }^{18} \mathrm{~F}\right]$ FEDAC-PET is a powerful tool for visualizing TSPO and monitoring the progress of various inflammatory diseases of the peripheral system. The present studies will contribute to determination of the pathogenic progress and will be of use in evaluating the therapeutic effects of antiinflammatory drugs. In future studies, we will perform or are performing PET imaging with $\left[{ }^{18} \mathrm{~F}\right] \mathrm{FEDAC}$ or other TSPO radiotracers to detect various inflammation in humans.

\section{Acknowledgements}

The author thanks Mr. Kumata, Ms. Hatori, Dr. Xie, Dr. Yanamoto, and Dr. Yamasaki in my group for their fruitful experiments and continuous outputs which contributed to this review.

\subsection{Role of Microglia in Neuroinflammation}

Hiromi Suzuki and Makoto Sawada

Abstract At present, common diseases of CNS were often chronic and progressive which could affect the patient's quality of life seriously. But the pathogenesis for most of them had not been fully understood and was no effective prevention or treatment. Certain pathological injury could cause cellular inflammatory response. Microglia, the first activated effector cells, played an important role in the immune defense process. Microglia had a two-way effect. On the one hand, activated microglia phagocytosed damaged cell debris and removed antigenic substances, and could release cytotoxic factors which would aggravate the injury of neuron. On the other hand, activated microglia may accumulate around damaged neurons and might induce neurotrophin-dependent protective activity. Therefore, to study the mechanism of microglia in the diseases of CNS and limit their effects on neuronal injury may help to retard the procession of some 
chronic disease and enhance the therapeutic effect of acute CNS diseases. Microglia are expected to become a new target for the treatment of neurodegenerative diseases and central nervous system diseases.

Keywords: Microglia, Subtype, Cytokine, Toxic change, Neuroprotection

\section{Abbreviations}

dbcAMP dibutyryl cyclic AMP

GM-CSF granulocyte-macrophage colony-stimulating factor

M-CSF macrophage colony-stimulating factor

MHC major histocompatibility complex

TGF transforming growth factor

\subsubsection{Introduction}

Neurodegenerative diseases such as Alzheimer's disease and Parkinson's disease cause neuroinflammation including expression of MHC antigens and production of inflammatory cytokines. However, the condition changes greatly in relation to the onset and the formation of a pathological condition. Microglia, macrophage-like cells in the central nervous system (CNS), are multifunctional cells; they play important roles not only in the neuro-inflammation but also in the development, differentiation, and maintenance of neural cells via their phagocytic activity and production of enzymes, cytokines, and trophic factors [148].

\subsubsection{Microglia Diversity}

Microglial cells show a rather uniform distribution of cell numbers throughout the brain with only minor population in some brain regions. Their in situ morphologies, however, may vary markedly from elongated forms observed in apposition with neuronal fibers to spherical cell bodies with sometimes extremely elaborated branching. Human fetal microglia display heterogeneity in phenotype identified by CD68 [149]. This heterogeneity gave rise to the hypothesis that these cells are differentially conditioned by their microenvironment and, therefore, also display specific patterns of differential gene expression. For example, mRNAs of TNFalpha, CD4, and Fc gamma receptor II are differentially expressed in microglia isolated from different area of the brain [150]. It has been reported that production of IL-12 [151] and histamine [152] are different in the subtypes of microglia. Furthermore, the subtypes of microglia show distinct acidification profiles by treatment with pepstatin A [153] and selective clearance of oligomeric beta-amyloid peptide [154]. The distinct phenotypes in activated forms of microglia might result from this heterogeneity.

Although the origin of microglia is still controversial, resident microglia is thought to be of mesodermal origin, being derived from a bone marrow precursor cell [155]. These cells or a lineage related to monocytes and macrophages may invade the CNS at an early embryonic stage to give rise eventually to typical process-bearing resident microglia [155]. Like macrophages in other tissues, the growth and activation of microglia is regulated by macrophage colony-stimulating factor (M-CSF) [156]. However, unlike macrophages, microglia are observed in macrophagedeficient mice [157-159], suggesting the possibility of the presence of M-CSF-independent populations.

Despite the postulate that microglia arise from blood monocytes, microglia appear to display phenotypes differing from macrophages. In vivo, microglia do not normally express MHC class I and II [160], CD14 [161], or scavenger receptor I and II [162]. Their appearance takes a ramified form [160]. In contrast, monocytes and macrophages express these surface antigens absent in microglia and their shape is ameboidal [160]. Like their in vivo counterparts, isolated microglia also differ from macrophages. Although cultured microglia are ameboidal, they can transform to a ramified form [163]. Microglial cell proliferation is induced by conditioned medium from astrocyte-enriched cultures [163]. Microglial production of interleukin (IL)-5 is stimulated by bioactive phorbol ester but not by interferon- $\gamma$, while the opposite is there for macrophages [164]. Microglia do not constitutively express IL-2 receptors under normal culture conditions, as do monocytes and macrophages [165]. Microglia express these receptors only after stimulation such as with lipopolysaccharide (LPS) [165]. IL-2 increases viability and proliferation of lipopolysaccharide-stimulated microglia [165], while macrophages respond to IL-2 by expressing IL-1, IL-6, and tumor necrosis factor $\alpha$ mRNAs, peroxide production, and microbicidal and tumoricidal activity [166, 167]. LPS, cyclosporin A, and FK506 downregulate expression of CD4 in microglia, but not in macrophages [168]. Finally, bone marrow chimera experiments have shown that resident microglia form highly stable pool of CNS cells displaying very little exchange with the bone marrow compartment [169-171]. These controversial observations suggest that microglia in the brain may be different from macrophages.

Recently, we identified two distinct subpopulations of microglia in the normal mouse brain: type I and type II. Both types had similar phagocytic activity, but they showed distinct phenotypes such as cell-surface markers, mRNA expression, and growth factor dependency. Type I, but not type II, microglia expressed the hallmarks of microglia such as CD14 and C3b receptor, and production of interleukin 
$1 \beta$. Type II microglia expresse surface markers for immature bone marrow cells, such as antigens against ER-MP12 and ER-MP20 antibodies, which are not detected in mature monocyte/macrophages. In addition, it also express onetenth amount of mature cell markers compared to monocytes/macrophages. Therefore, type II microglia may arise from progenitor cells similar to immature bone marrow cells which migrate to the CNS during the early developmental stages then differentiate in the CNS; monocytedifferentiation out of bone marrow like extrathymic differentiation of T-cells [172]. This suggests that the two distinct type of microglia arise from distinct origins. In addition, type I microglia exhibited M-CSF-dependent growth, while the type II were M-CSF-independent. A previous report suggested that alveolar macrophages are GM-CSF- but not M-CSF-dependent [173]. These observations indicate that there are two distinct populations of microglia which are probably of different origins and have different functions in the brain.

\subsubsection{Microglia and Neuroinflammation}

The presence of oxidative stress and inflammatory activity is one of the significant pathological features of neuroinflammatory diseases $[174,175]$. It has been shown that the levels of cytokines such as tumor necrosis factor (TNF)- $\alpha$, interleukin (IL)- $1 \beta$, and interferon (IFN)- $\gamma$ are elevated in the substantia nigra of patients with Parkinson's disease [176]. Since microglia are a principal source of these cytokines, the data support microglial involvement in the pathogenesis of neuroinflammatory diseases. However, the role of activated microglia is controversial. For example, the characteristic pathological features of the Parkinson's disease brain are a selective and progressive loss of dopamine neurons of the substantia nigra and their terminals in the caudate-putamen, along with focal accumulation of activated microglia in the substantia nigra and caudate-putamen. The traditional view is that microglia act merely as scavengers and their activation is secondary to the neuronal damage. However, activated microglia have been observed in the limbic system and neocortex, where there are few or no degenerating neurons, in significantly higher numbers in PD brains than in brains from normal controls [177].

Activation of microglia has also been identified in the substantia nigra and/or striatum of parkinsonian animal models, such as 1-methyl-4-phenyl-1,2,3,6tetrahydropyridine (MPTP)-induced parkinsonism [178, 179]. The link between microglia activation and selective neuronal vulnerability has led many researchers to suggest that microglia activity participates in neuronal demise. In this respect, microglial cytotoxicity may contribute to or even promote neuronal damage. Activated microglia are capable of releasing numerous cytotoxic agents, including proteolytic enzymes, cytokines, complement proteins, reactive oxygen intermediates, NMDA-like toxins, and nitric oxide [148]. In fact, $\beta$-amyloid, the senile plaquederived component found in Alzheimer's disease, appears to elicit neurotoxic responses through the activation of microglia [180]. However, this suggestion relies solely on in vitro data and as yet no evidence has been presented that indicates that activated microglia destroy neurons under in vivo conditions.

\subsubsection{Existence of Nontoxic Activated Form of Microglia in the Brain}

Recently we showed that highly activated microglia treated with LPS may have neurotrophic potential toward dopamine neurons in neonatal mice administered MPTP [181]. Tyrosine hydroxylase activity and the levels of dopamine and dihydroxyphenylacetic acid (DOPAC), as well as those of the pro-inflammatory cytokines IL- $1 \beta$ and IL-6, were elevated in the midbrain of LPS- and MPTP-treated neonatal mice. The viability of dopamine (A9) neurons was preserved in neonatal mice of the LPS-MPTP group compared with the MPTP group. In contrast, the viability of these neurons in aged mice dropped significantly. These results may suggest that activated microglia show different phenotypes; i.e., microglia activated by LPS in the neonatal brain may be neuroprotective for dopaminergic neurons in MPTP-treated mice, whereas in aged mice they may be neurotoxic for dopaminergic neurons. The dissociation between injury-induced microglial activation and neuronal degeneration in TNF receptor and colony-stimulating factor 1 (CSF-1) knockout mice suggests that microglial activation is not a determinate event in dopaminergic neuronal damage in brain. Furthermore, there is a growing body of evidence that microglia may play a beneficial role in ischemia by secreting factors (growth factors or cytokines) that promote survival of neurons. Therefore, activated microglia may produce not only neurotoxic effects but also neuroprotective ones, depending upon their environmental situation.

\subsubsection{Direct Evidence of Neuroprotection by Microglia in the Brain}

We have reported that exogenous microglia enter the brain parenchyma through the blood-brain barrier and migrate to ischemic hippocampal lesions when they are injected into 
the circulation. By applying this brain-targeted delivery technique, we investigated the effect of exogenous microglia on ischemic pyramidal neurons [182]. To this end, we isolated microglia from neonatal mixed brain cultures, labeled them with a fluorescent dye PKH26, and injected them into the artery of Mongolian gerbils subjected to ischemia reperfusion neuronal injury. PKH26-labeled microglia migrated to the ischemic hippocampal lesion and increased the survival of neurons, even when the cells were injected $24 \mathrm{~h}$ after the ischemic insult. Furthermore, stimulation of isolated microglia with IFN- $\gamma$ enhanced the neuroprotective effect on the ischemic neurons.

Microglia also protected ischemia-induced learning disability. As migratory microglia increased the expression of brain-derived neurotrophic factor (BDNF) and glial cell linederived neurotrophic factor (GDNF) in the ischemic hippocampus, they might induce neurotrophin-dependent protective activity in damaged neurons. These results represent the first experimental demonstration of neurotrophic effects of microglia on transient global ischemia in vivo. Since peripherally injected microglia exhibit specific affinity for ischemic brain lesions and protect against ischemic neuronal injury in the present model, we suggest that microglia may have the potential to be used as a candidate for cell therapy for CNS repair following transitory global ischemia and other neurodegenerative diseases.

\subsubsection{Toxic Transformation of Microglia by Nef Protein Introduction}

Nef, a multifunctional HIV protein, activates the Vav/Rac/ p21-activated kinase (PAK) signaling pathway. Given the potential role of this pathway in the activation of the phagocyte NADPH oxidase, we have investigated the effect of the HIV-1 Nef protein on microglia superoxide production and toxicity for neurons [183]. Microglia were transduced with lentiviral expression vectors to produce a high level of Nef protein. Expression of Nef did not activate the NADPH oxidase by itself, but led to a massive enhancement of the responses to a variety of stimuli $(\mathrm{Ca} 2+$ ionophore, formyl peptide, endotoxin) and induction to produce a large amount of superoxide. These effects were not caused by upregulation of phagocyte NADPH oxidase subunits. Nef mutants lacking motifs involved in the interaction with plasma membrane and/or other cytosolic proteins failed to reproduce the effects of wild-type Nef, suggesting involvement of a certain signaling pathway in microglia for their trophic-toxic control. Our results suggest a key role for Rac activation in the priming for microglia toxic activation, which is enhanced by Nef introduction in the nontoxic form of microglia. Rac activation is not sufficient to induce the toxic form of microglia accompanied by stimulation of the phagocyte NADPH oxidase; however, it markedly enhances the NADPH oxidase response to other stimuli and might be involved in the trophic-toxic control of microglia.

\subsubsection{CNS Cytokine Network}

Cytokines are polypeptidic soluble factors that control the growth and differentiation of cells involved in immune and hematopoietic systems. These factors were initially considered to act on target cells in a cell-type and stage-specific manner. However, it has been shown that their biological actions are pleiotropic, complementary, and counteractive; each of them exerts multiple effects on different cells, and different factors can act on the same cell populations to induce similar or opposite effects. Moreover, production of several cytokines is controlled by other cytokines in a stimulatory or inhibitory manner, and, in some cases, acts as a cascade. These complex cytokine actions are therefore referred to as a cytokine network.

Recent evidence suggests that bidirectional communication occurs between cells of the nervous and immune systems. The basis for this communication is the release of cytokines by immune component cells, as well as hormone products of the neuroendocrine system. Cells resident within the CNS can synthesize, secrete, and respond to inflammatory cytokines not only contributing to the responses to injury or immunological challenge within the CNS but also regulating their own growth and differentiation potential. There are many similarities of cytokine actions between the immune system and the CNS. However, there are also several unique modes of cytokine action in the CNS (i.e., their target cells, inducing functions on the target cells such as other cytokine production, major histocompatibility complex (MHC) antigen induction, and cell growth control, and inducing agents of their production). Therefore, the actions and communication of cytokines in the CNS are designated as the CNS cytokine network [184].

Microglia are one of the most important cells in the CNS cytokine network. They produce a variety of cytokines, as well as their proliferation and other functions are regulated by cytokines. Astrocytes are another cell population important in the CNS cytokine network. There are many similar aspects of cytokine production and responses between microglia and astrocytes; however, the roles in the CNS cytokine network may differ between these cells; microglia may have an important function in the removal of dead cells or their remnants by phagocytosis in brain injury, while astrocytes provide structural and environmental support for neurons. There are no clear indications of the difference between 
the roles of microglia and astrocytes in the cytokine production and response.

Some of these cytokines control the growth, differentiation, and activation of cells in the CNS, and some modulate growth factor production, MHC antigen expression, and morphological changes. Their biological functions are mediated by specific receptors, some of which are expressed in the CNS, and cellular localization of cytokine receptors in the CNS has been demonstrated. Neuronal cells and oligodendrocytes as well as microglia and astrocytes expressed several cytokine receptors indicating that certain functions of these cells may also be controlled by some cytokines. However, to determine the involvement of cytokines in controlling the functions and development of CNS cells requires elucidating the functional relationships of cytokines and these cells.

There are two aspects of roles of the CNS cytokine network, one of which is regulation of growth and differentiation of neural cells, and production of cytokines in the CNS. Another aspect of the CNS cytokine network is its interaction with the immune system. Since the majority of cytokines are produced by immune cells, it is possible that immune cells may also control the growth and function of CNS cells. Therefore, the control of microglial growth by cytokines is an important method of regulation of CNS cells by peripheral immune cells. In addition to this, cytokines produced in the CNS can control immune cell functions. These communications may be important for brain-immune interactions, and some aspects may play crucial roles in certain pathological conditions such as AIDS-dementia complex and multiple sclerosis as well as several neurological diseases.

\subsection{Application of Preclinical PET Imaging for Infectious Diseases}

\section{Kodai Nishi and Takashi Kudo}

\begin{abstract}
PET is a useful imaging tool for basic studies. Preclinical imaging studies such as small-animal PET allow the performance of imaging experiments using the same individuals, without sacrificing the animals. Some researchers who study infectious diseases are thus paying close attention to preclinical imaging techniques. Generally, pathogens are classified by their risk, as the biosafety level (BSL). When conducting research using high-BSL pathogens, special facilities are necessary for pathogen containment. At Nagasaki University, a small-animal imaging system was introduced into BSL-3 section, and various infectious disease imaging studies have been conducted, such as for severe fever with thrombocytopenia syndrome (SFTS), leishmani-
\end{abstract}

asis, and so on. FDG and ${ }^{67} \mathrm{Ga}$-citrate are widely used radiotracers for the clinical imaging that can detect inflammation due to infection. For animal imaging, ${ }^{68} \mathrm{Ga}$-citrate is a PET tracer that is not yet approved for human imaging, and can be used instead of ${ }^{67} \mathrm{Ga}$-citrate, which is single photon emission computed tomography (SPECT) tracer. Even without using special or unorthodox radiotracers, new knowledge and important information can be obtained from preclinical PET studies.

Keywords: Small animal imaging, Preclinical study, Infectious diseases, Biosafety level

\subsubsection{Introduction}

PET imaging has recently gained wide use not only in clinical stages but also in basic studies. Small-animal imaging uses the same technology as clinical imaging. Clinical research and problems can thus be introduced to preclinical research directly using small animals. Conversely, applying results obtained from preclinical studies to clinical imaging is also possible. Imaging has bridged the gap between basic studies and clinical research, and has become widely recognized as a tool for translational research.

In traditional animal experiments, relatively high numbers of animals are often required. One reason is that animals are sacrificed to remove organs or tissues for the purposes of observation and measurement. Another is the influence of individual differences. In particular, the four elements of pharmacokinetics (absorption, distribution, metabolism, and excretion) are known to be strongly affected by individual differences. The number of samples must therefore be increased for accurate statistical processing.

On the other hand, preclinical imaging experiments allow use of the same individual, performing the study and repeatedly acquiring data without killing the individual. This means that applying human imaging techniques to a small animal allows follow-up of changes over time in disease models in the same individual, clarifying changes according to the disease condition more precisely, as in daily practice in the clinical field. In addition, preclinical imaging is an effective technique not only for providing biological information but also for reducing the number of experimental animals.

In the area of research into infectious diseases, evaluation of pathogen infection, tracking disease state, and observation over time of individuals are very important, because infectious and inflammatory diseases change with the passage of time. For example, diseases may pass through stages of infection, pathogenesis, ingravescence, remission, and healing. When those stages of infectious disease are studied by sacrificing the animals, the number of animals required to obtain meaningful results will become extremely large due to the large interindi- 
Table 1.2 BSL classification of representative pathogens

\begin{tabular}{|c|c|c|c|c|}
\hline BSL & Virus & Bacteria & Fungi & Parasitic insect \\
\hline \multirow[t]{4}{*}{2} & Dengue virus & C. botulinum & Aspergillus fumigatus & Balantidium coli \\
\hline & Hepatitis $C$ virus & C. jejuni, coli & Candida albicans & Leishmania \\
\hline & Norovirus & E. coli & Cryptococcus neoformans & Echinococcus \\
\hline & Papillomavirus & H. pylori & & Anisakinae \\
\hline \multirow[t]{4}{*}{3} & SARS corona virus & F. tularensis & Blastomyces dermatitidis & \multirow[t]{4}{*}{ N/A } \\
\hline & West-Nile virus & M. tuberculosis & Histoplasma capsulatum & \\
\hline & SFTS virus & S. enterica & Penicillium marneffei & \\
\hline & New York virus & R. japonica & & \\
\hline \multirow[t]{3}{*}{4} & Lassa & \multirow[t]{3}{*}{ N/A } & \multirow[t]{3}{*}{ N/A } & \multirow[t]{3}{*}{ N/A } \\
\hline & Ebola & & & \\
\hline & Marburg & & & \\
\hline
\end{tabular}

vidual variance of animals. Small animal PET imaging has therefore gained attention as a useful experimental method.

\subsubsection{Imaging Laboratory for Infectious Diseases}

To conduct imaging studies using infected model animals, a special laboratory is required. This is because researchers must be protected not only from radiation exposure but also from the risk of infection. Biosafety level (BSL) is a classification depending on the degree of pathogen risk. Table 1.2 shows representative pathogens sorted by BSL from the guidelines of the National Institute of Infectious Disease (NIID).

When dealing with high-risk pathogens, researchers need to use a biocontainment laboratory conforming to the World Health Organization (WHO) guidelines. When using imaging systems, measures must also be taken to avoid contamination by biohazards, such as installation in separated safety rooms, prevention of cross-contamination using sealed animal tubes, and so on [185]. In addition, researchers need to wear a Tyvek coverall, mask, rubber gloves and face guard (Fig. 1.29). Given the restrictions as described above, only limited numbers of facilities around the world are capable of performing imaging studies for infectious diseases, and relatively few studies have been conducted.

\subsubsection{PET Imaging with Infectious Small Animals}

In 2012, a small-animal imaging system was introduced to the BSL-3 section of the radioisotope center at Nagasaki University, making this the only facility in which researchers could perform imaging of infectious diseases requiring a high BSL in Japan. Since then, various infectious diseases imaging studies have been conducted.

Hayasaka et al. reported an FDG-PET study using mice infected with severe fever with thrombocytopenia syndrome

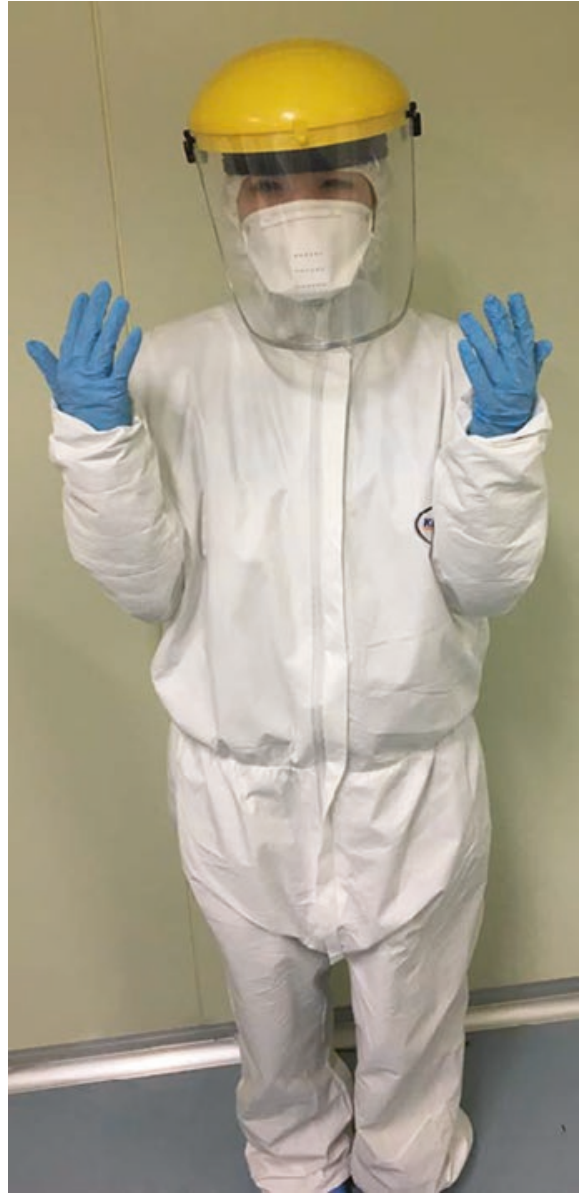

Fig. 1.29 An example of using protective clothing to prevent exposure of the skin and cover the face completely with the face guard

(SFTS) virus, which is categorized as BSL-3. SFTS is a tickborne infection that causes severe inflammation in the gastrointestinal tract [186]. In that study, SFTSV-infected mice were administered about $10 \mathrm{MBq}$ of FDG intravenously via the tail vain. PET acquisition was performed for $30 \mathrm{~min}$ from $30 \mathrm{~min}$ after FDG injection. FDG uptake was found along the course of the intestine in the SFTS-infected mouse (Fig. 1.30) [187]. 
PET studies on SFTS have also been performed using ${ }^{68} \mathrm{Ga}$ tracer. Fuchigami et al. tried to visualize SFTS with ${ }^{68} \mathrm{Ga}$-citrate [188]. Generally, ${ }^{67} \mathrm{Ga}$-citrate is a radiotracer used for single-photon computed tomography (SPECT), and is widely used to detect tumors [189, 190], inflammation, and infectious diseases [190, 191]. Since ${ }^{68} \mathrm{Ga}$-citrate is a PET tracer behaving exactly the same as ${ }^{67} \mathrm{Ga}$-citrate, targets can be imaged quantitatively and with higher sensitivity. In that study, SFTSV-infected mice were administered about $5 \mathrm{MBq}$ of ${ }^{68} \mathrm{Ga}$-citrate intravenously via the tail vain. PET acquisition was performed for $1 \mathrm{~h}$, starting $2 \mathrm{~h}$ after ${ }^{68} \mathrm{Ga}$-citrate injection. As shown in Fig. 1.31, high accumulation of ${ }^{68} \mathrm{Ga}$-citrate in the gastrointestinal tract was observed in SFTSV-infected mice, and the accumulation pattern of ${ }^{68} \mathrm{Ga}$-citrate resembled that of FDG.

In addition, ${ }^{68} \mathrm{Ga}$-citrate was used for PET imaging of leishmaniasis [188]. Leishmaniasis is a tropical disease caused by Leishmania parasites categorized as BSL-2, and Leishmania leads to strong localized inflammation [192]. Leishmania parasite-infected mice were administered about $4 \mathrm{MBq}$ of ${ }^{68} \mathrm{Ga}$-citrate intravenously via the tail vein. PET acquisition was performed for $1 \mathrm{~h}$, starting $2 \mathrm{~h}$ after ${ }^{68} \mathrm{Ga}$-citrate injection. As shown in Fig. 1.32, inflammation site-specific accumulation of ${ }^{68} \mathrm{Ga}$-citrate was observed [188].

The examples given here are only a small selection of many studies underway at Nagasaki University. Infectious disease studies are more diverse than tumor studies, because infectious diseases have numerous diseases classified as tropical infections and emerging infectious diseases, and many issues have not been elucidated for each disease. FDG and ${ }^{68} \mathrm{Ga}$-citrate used in the studies introduced this section are widely used general radiotracers that are easily obtained. Even without using special or unorthodox radiotracers, new knowledge can be obtained from preclinical PET studies.
Fig. 1.30 FDG-PET/CT images of mice with or without SFTSV infection. In the mock-infected mouse, FDG accumulation is observed in the heart and bladder. On the other hand, in the SFTSV-infected mouse, FDG accumulates in the gastrointestinal tract (yellow arrow)
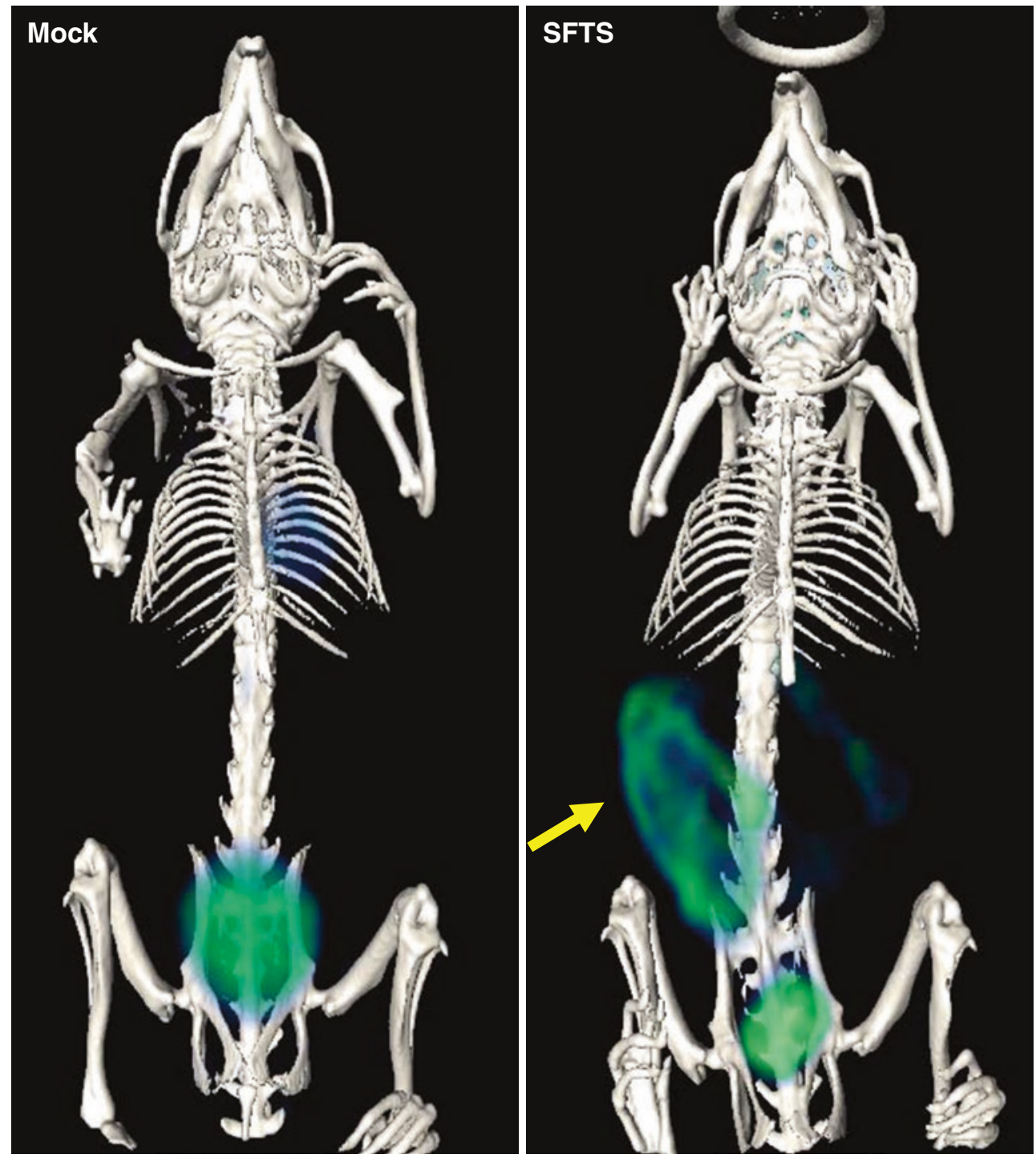


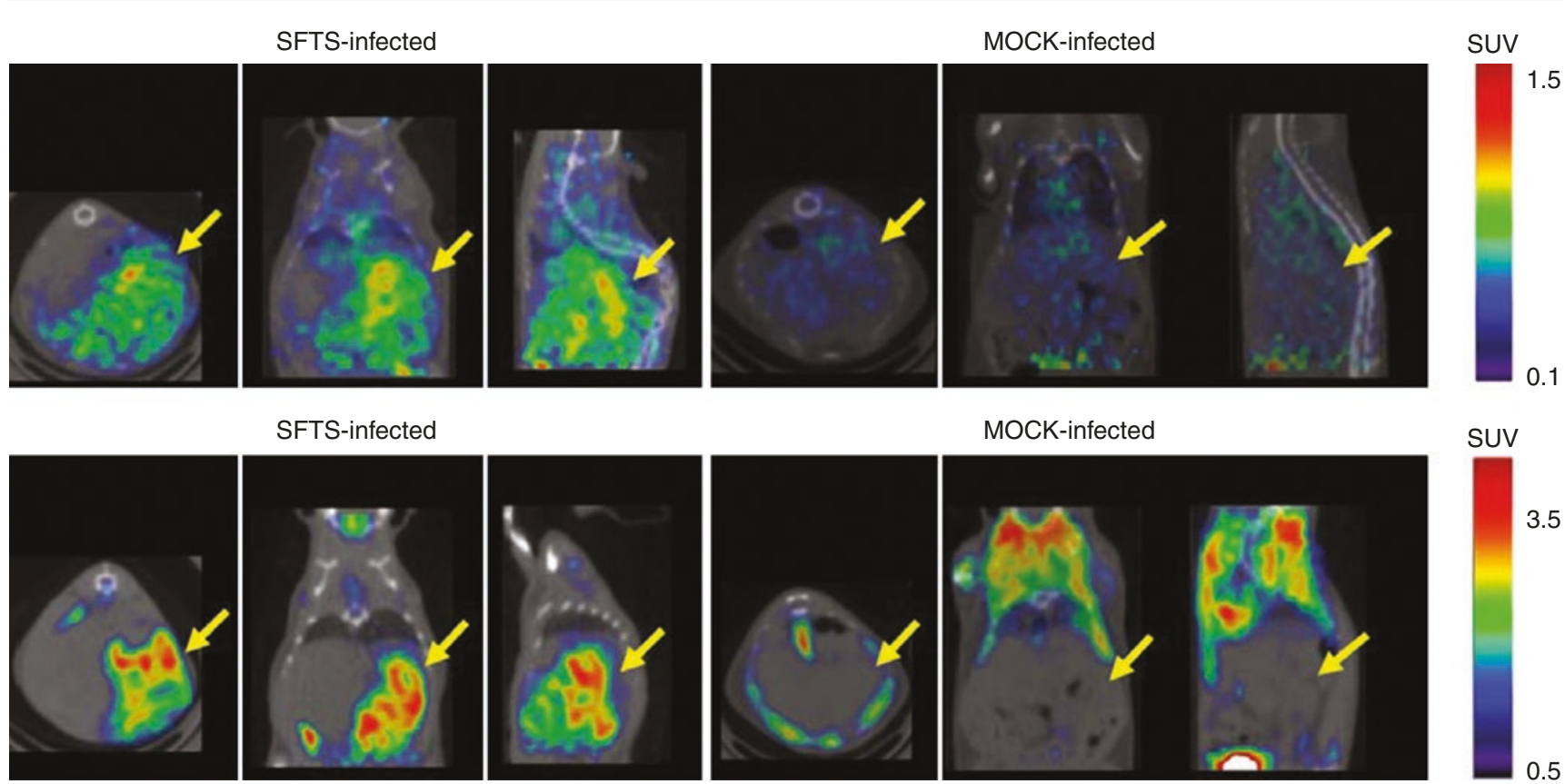

Fig. 1.31 Images from ${ }^{68} \mathrm{Ga}$-citrate (a) and FDG (b) PET/CT of SFTSV-infected and mock-infected mice. Yellow arrows indicate the gastrointestinal tract

The pathological condition of the infected animal will change depending on species differences and the infected pathogen. Some models only have about 3 days between infection and death. When conducting imaging research into infectious diseases, investigation of the survival rate of model animals as a preliminary experiment and preparation of a survival curve is desirable. Also, breeding of model animals requires prevention of infection with other infectious diseases. Experiments using infected animals thus require some additional caution, as previously mentioned.

In conclusion, small animal imaging using preclinical PET bridges the gap between basic researches and clinical practices and works as an important tool for translational research in infectious disease.

\section{Acknowledgement}

The authors are grateful to the American Chemical Society (ACS) for allowing reuse of some images (Figs. 1.31 and 1.32) and a direct link to the ACS article: https://pubs.acs. org/doi/abs/10.1021/acsomega.7b00147.

\section{References}

1. Kubota K. From tumor biology to clinical PET: a review of positron emission tomography (PET) in oncology. Ann Nucl Med. 2001;15:471-86.

2. Fletcher JW, Djulbegovic B, Soares HP, et al. Recommendations on the use of 18F-FDG PET in oncology. J Nucl Med. 2008;49:480-508.

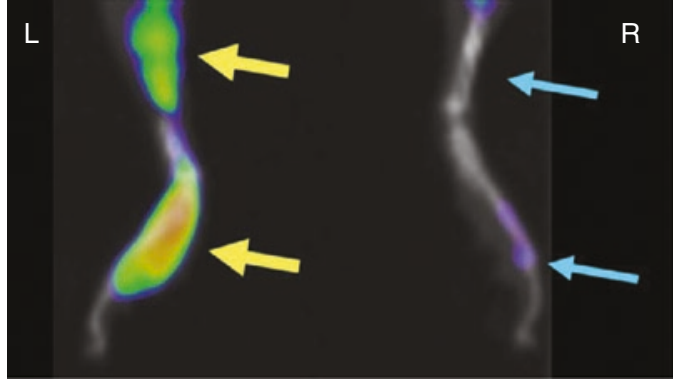

SUV

Fig. 1.32 PET/CT images of footpad regions in L. major-infected mice. A signal from the ${ }^{68} \mathrm{Ga}$-citrate is observed at a site in the left leg (yellow arrows), with no signal in the contralateral leg (blue arrows)

3. Boellaard R, Delgado-Bolton R, Oyen WJ, et al. FDG PET/CT: EANM procedure guidelines for tumour imaging: version 2.0. Eur J Nucl Med Mol Imaging. 2015;42:328-54.

4. Tahara T, Ichiya Y, Kuwabara Y, et al. High [18F]fluorodeoxyglucose uptake in abdominal abscess: a PET study. J Comput Assist Tomogr. 1989;13:829-31.

5. Sasaki M, Ichiya Y, Kuwabara Y, et al. Ring-like uptake of [18F] FDG in brain abscess: a PET study. J Comput Assist Tomogr. 1990;14:486-7.

6. Kubota K, Matsuzawa T, Fujiwara T, et al. Differential diagnosis of lung tumor with positron emission tomography: a prospective study. J Nucl Med. 1990;31:1927-33.

7. Kubota R, Yamada S, Kubota K, et al. Intratumoral distribution of fluorine-18-fluorodeosxyglucose in vivo: high accumulation in macrophages and granulation tissues studied by microautoradiography. J Nucl Med. 1992;33:1972-80.

8. Kubota K, Kubota R, Yamada S. FDG accumulation in tumor tissue. J Nucl Med. 1993;34:75-82. 
9. Yamada S, Kubota K, Kubota R, et al. High accumulation of fluorine-18-fluorodeoxyglucose in turpentine-induced inflammatory tissue. J Nucl Med. 1995;36:1301-6.

10. Mochizuki T, Tsukamoto E, Kuge Y, et al. FDG uptake and glucose transporter subtype expressions in experimental tumor and inflammation models. J Nucl Med. 2001;42:1551-5.

11. Zhao S, Kuge Y, Masashi Kohanawa M, et al. Usefulness of $11 \mathrm{C}$-Methionine for differentiating tumors from granulomas in experimental rat models: a comparison with $18 \mathrm{~F}-\mathrm{FDG}$ and $18 \mathrm{~F}$ FLT. J Nucl Med. 2008;49:135-41.

12. Deichen TJ, Prante O, Gack M, et al. Uptake of [18F]fluorodeoxyglucose in human monocyte-macrophages in vitro. Eur J Nucl Med. 2003;30:267-73.

13. Malide D, Davies-Hill TM, Levine M, et al. Distinct localization of GLUT-1, -3 , and -5 in human monocyte-derived macrophages: effects of cell activation. Am J Phys. 1998;274(3):E516-26.

14. Jones HA, Cadwallader KA, White JF, et al. Dissociation between respiratory burst activity and deoxyglucose uptake in human neutrophil granulocytes: implications for interpretation of 18F-FDG PET images. J Nucl Med. 2002;43:652-7.

15. Ishimori T, Saga T, Mameda M, et al. Increased 18F-FDG uptake in a model of inflammation: Concanavalin A-mediated lymphocyte activation. J Nucl Med. 2002;43:658-63.

16. Lee DM, Weinblatt ME. Rheumatoid arthritis. Lancet. 2001;358:903-11.

17. Matsui T, Nakata N, Nagai S, et al. Inflammatory cytokines and hypoxia contribute to $18 \mathrm{~F}-\mathrm{FDG}$ uptake by cells involved in pannus formation in rheumatoid arthritis. J Nucl Med. 2009;50:920-6.

18. Roiniotis J, Dinh H, Masendycz P, et al. Hypoxia prolongs monocyte/macrophage survival and enhanced glycolysis is associated with their maturation under aerobic conditions. J Immunol. 2009;182:7974-81.

19. Garcia-Carbonell R, Divakaruni AS, Lodi A, et al. Critical role of glucose metabolism in rheumatoid arthritis fibroblast-like synoviocytes. Arthritis Rheumatol. 2016;68:1614-26.

20. Cramer T, Yamanishi Y, Clausen BE, et al. HIF-1 $\alpha$ is essential for myeloid cell-mediated inflammation. Cell. 2003;112:645-57.

21. Denko NC. Hypoxia, HIF1 and glucose metabolism in the solid tumour. Nat Rev Cancer. 2008;8:705-13.

22. Jamar F, Buscombe J, Chiti A, et al. EANM/SNMI guideline for $18 \mathrm{~F}-\mathrm{FDG}$ use in inflammation and infection. J Nucl Med. 2013;54:647-58.

23. Lendon CL, Davies MJ, Born GV, et al. Atherosclerotic plaque caps are locally weakened when macrophages density is increased. Atherosclerosis. 1991;87:87-90.

24. MacNeill BD, Jang IK, Bouma BE, et al. Focal and multi-focal plaque macrophage distributions in patients with acute and stable presentations of coronary artery disease. J Am Coll Cardiol. 2004;44:972-9.

25. Kubota R, Kubota K, Yamada S, et al. Microautoradiographic study for the differentiation of intratumoral macrophages, granulation tissues and cancer cells by the dynamics of fluorine-18fluorodeoxyglucose uptake. J Nucl Med. 1994;35:104-12.

26. Tatsumi M, Cohade C, Nakamoto Y, et al. Fluorodeoxyglucose uptake in the aortic wall at PET/CT: possible finding for active atherosclerosis. Radiology. 2003;229:831-7.

27. Rudd JH, Warburton EA, Fryer TD, et al. Imaging atherosclerotic plaque inflammation with $[18 \mathrm{~F}]$-fluorodeoxyglucose positron emission tomography. Circulation. 2002;105:2708-11.

28. Yun M, Jang S, Cucchiara A, et al. 18F FDG uptake in the large arteries: a correlation study with the atherogenic risk factors. Semin Nucl Med. 2002;32:70-6.

29. Ogawa M, Ishino S, Mukai T, et al. (18)F-FDG accumulation in atherosclerotic plaques: immunohistochemical and PET imaging study. J Nucl Med. 2004;45:1245-50.
30. Graebe M, Pedersen SF, Borgwardt L, et al. Molecular pathology in vulnerable carotid plaques: correlation with [18]-fluorodeoxyglucose positron emission tomography (FDG-PET). Eur J Vasc Endovasc Surg. 2009;37:714-21.

31. Tawakol A, Migrino RQ, Bashian GG, et al. In vivo 18F-fluorodeoxyglucose positron emission tomography imaging provides a noninvasive measure of carotid plaque inflammation in patients. J Am Coll Cardiol. 2006;48:1818-24.

32. Wu YW, Kao HL, Chen MF, et al. Characterization of plaques using 18F-FDG PET/CT in patients with carotid atherosclerosis and correlation with matrix metalloproteinase-1. J Nucl Med. 2007;48:227-33.

33. Tahara N, Kai H, Yamagishi S, et al. Vascular inflammation evaluated by $[18 \mathrm{~F}]$-fluorodeoxyglucose positron emission tomography is associated with the metabolic syndrome. J Am Coll Cardiol. 2007;49:1533-9.

34. Skeoch S, Williams H, Cristinacce P, et al. Evaluation of carotid plaque inflammation in patients with active rheumatoid arthritis using (18)F-fluorodeoxyglucose PET-CT and MRI: a pilot study. Lancet. 2015;385(Suppl 1):S91.

35. Ogawa M, Nakamura S, Saito Y, et al. What can be seen by $18 \mathrm{~F}-$ FDG PET in atherosclerosis imaging? The effect of foam cell formation on 18F-FDG uptake to macrophages in vitro. J Nucl Med. 2012;53:55-8.

36. Ishino S, Ogawa M, Mori I, et al. 18F-FDG PET and intravascular ultrasonography (IVUS) images compared with histology of atherosclerotic plaques: 18F-FDG accumulates in foamy macrophages. Eur J Nucl Med Mol Imaging. 2014;41:624-33.

37. Colin S, Chinetti-Gbaguidi G, Staels B. Macrophage phenotypes in atherosclerosis. Immunol Rev. 2014;262:153-66.

38. Mantovani A, Garlanda C, Locati M. Macrophage diversity and polarization in atherosclerosis: a question of balance. Arterioscler Thromb Vasc Biol. 2009;29:1419-23.

39. Satomi T, Ogawa M, Mori I, et al. Comparison of contrast agents for atherosclerosis imaging using cultured macrophages: FDG versus ultrasmall superparamagnetic iron oxide. J Nucl Med. 2013;54:999-1004.

40. Shiomi M, Ito T, Hirouchi Y, et al. Fibromuscular cap composition is important for the stability of established atherosclerotic plaques in mature WHHL rabbits treated with statins. Atherosclerosis. 2001;157:75-84.

41. Fukumoto Y, Libby P, Rabkin E, et al. Statins alter smooth muscle cell accumulation and collagen content in established atheroma of watanabe heritable hyperlipidemic rabbits. Circulation. 2001;103:993-9.

42. Daugherty A, Zweifel BS, Schonfeld G. The effects of probucol on the progression of atherosclerosis in mature Watanabe heritable hyperlipidaemic rabbits. Br J Pharmacol. 1991;103:1013-8.

43. Steinberg D, Parthasarathy S, Carew TE. In vivo inhibition of foam cell development by probucol in Watanabe rabbits. Am J Cardiol. 1988;62:6B-12B.

44. Ogawa M, Magata Y, Kato T, et al. Application of 18F-FDG PET for monitoring the therapeutic effect of antiinflammatory drugs on stabilization of vulnerable atherosclerotic plaques. J Nucl Med. 2006;47:1845-50.

45. Pirro M, Simental-Mendia LE, Bianconi V, et al. Effect of statin therapy on arterial wall inflammation based on 18F-FDG PET/CT: a systematic review and meta-analysis of interventional studies. $\mathrm{J}$ Clin Med. 2019;8:118.

46. Moghbel M, Al-Zaghal A, Werner TJ, et al. The role of PET in evaluating atherosclerosis: a critical review. Semin Nucl Med. 2018;48:488-97.

47. Mehta NN, Torigian DA, Gelfand JM, et al. Quantification of atherosclerotic plaque activity and vascular inflammation using [18F] fluorodeoxyglucose positron emission tomography/computed tomography (FDG-PET/CT). J Vis Exp. 2012;63:e3777. 
48. Wu YW, Kao HL, Huang CL, et al. The effects of 3-month atorvastatin therapy on arterial inflammation, calcification, abdominal adipose tissue and circulating biomarkers. Eur J Nucl Med Mol Imaging. 2012;39:399-407.

49. Ishii $\mathrm{H}$, Nishio M, Takahashi $\mathrm{H}$, et al. Comparison of atorvastatin 5 and $20 \mathrm{mg} / \mathrm{d}$ for reducing F-18 fluorodeoxyglucose uptake in atherosclerotic plaques on positron emission tomography/computed tomography: a randomized, investigator-blinded, open-label, 6-month study in Japanese adults scheduled for percutaneous coronary intervention. Clin Ther. 2010;32:2337-47.

50. Tahara N, Kai H, Ishibashi M, et al. Simvastatin attenuates plaque inflammation: evaluation by fluorodeoxyglucose positron emission tomography. J Am Coll Cardiol. 2006;48:1825-31.

51. Fayad ZA, Mani V, Woodward M, et al. Safety and efficacy of dalcetrapib on atherosclerotic disease using novel non-invasive multimodality imaging (dal-PLAQUE): a randomised clinical trial. Lancet. 2011;378:1547-59.

52. Duivenvoorden R, Mani V, Woodward M, et al. Relationship of serum inflammatory biomarkers with plaque inflammation assessed by FDG PET/CT: the dal-PLAQUE study. JACC Cardiovasc Imaging. 2013;6:1087-94.

53. Hellberg S, Silvola JM, Kiugel M, et al. Type 2 diabetes enhances arterial uptake of choline in atherosclerotic mice: an imaging study with positron emission tomography tracer (1)(8) F-fluoromethylcholine. Cardiovasc Diabetol. 2016;15:26.

54. Kato K, Schober O, Ikeda M, et al. Evaluation and comparison of $11 \mathrm{C}$-choline uptake and calcification in aortic and common carotid arterial walls with combined PET/CT. Eur J Nucl Med Mol Imaging. 2009;36:1622-8.

55. Ammirati E, Moroni F, Magnoni M, et al. Carotid artery plaque uptake of (11)C-PK11195 inversely correlates with circulating monocytes and classical CD14(++)CD16(-) monocytes expressing HLA-DR. Int J Cardiol Heart Vasc. 2018;21:32-5.

56. Gaemperli O, Shalhoub J, Owen DR, et al. Imaging intraplaque inflammation in carotid atherosclerosis with 11C-PK11195 positron emission tomography/computed tomography. Eur Heart J. 2012;33:1902-10.

57. Lee R, Kim J, Paeng JC, et al. Measurement of (68)Ga-DOTATOC uptake in the thoracic aorta and its correlation with cardiovascular risk. Nucl Med Mol Imaging. 2018;52:279-86.

58. Malmberg C, Ripa RS, Johnbeck CB, et al. 64Cu-DOTATATE for noninvasive assessment of atherosclerosis in large arteries and its correlation with risk factors: head-to-head comparison with $68 \mathrm{Ga}$-DOTATOC in 60 patients. J Nucl Med. 2015;56:1895-900.

59. Mateo J, Izquierdo-Garcia D, Badimon JJ, et al. Noninvasive assessment of hypoxia in rabbit advanced atherosclerosis using (1)(8)F-fluoromisonidazole positron emission tomographic imaging. Circ Cardiovasc Imaging. 2014;7:312-20.

60. Dai D, Chuang HH, Macapinlac HA, et al. Correlation of fluorine 18-labeled sodium fluoride uptake and arterial calcification on whole-body PET/CT in cancer patients. Nucl Med Commun. 2019;40:604-10.

61. Kitagawa T, Yamamoto H, Nakamoto Y, et al. Predictive value of (18)F-sodium fluoride positron emission tomography in detecting high-risk coronary artery disease in combination with computed tomography. J Am Heart Assoc. 2018;7:e010224.

62. Bellinge JW, Francis RJ, Majeed K, et al. In search of the vulnerable patient or the vulnerable plaque: (18)F-sodium fluoride positron emission tomography for cardiovascular risk stratification. J Nucl Cardiol. 2018;25:1774-83.

63. Kitagawa T, Yamamoto H, Toshimitsu S, et al. (18)F-sodium fluoride positron emission tomography for molecular imaging of coronary atherosclerosis based on computed tomography analysis. Atherosclerosis. 2017;263:385-92.
64. Hellberg S, Silvola JMU, Liljenback H, et al. Amyloid-targeting PET tracer [(18)F]Flutemetamol accumulates in atherosclerotic plaques. Molecules. 2019;24:1072.

65. McKenney-Drake ML, Moghbel MC, Paydary K, et al. (18)F-NaF and (18)F-FDG as molecular probes in the evaluation of atherosclerosis. Eur J Nucl Med Mol Imaging. 2018;45:2190-200.

66. Li X, Heber D, Cal-Gonzalez J, et al. Association between osteogenesis and inflammation during the progression of calcified plaque evaluated by (18)F-fluoride and (18)F-FDG. J Nucl Med. 2017;58:968-74.

67. Quirce R, Martinez-Rodriguez I, Banzo I, et al. New insight of functional molecular imaging into the atheroma biology: 18F$\mathrm{NaF}$ and 18F-FDG in symptomatic and asymptomatic carotid plaques after recent CVA. Preliminary results. Clin Physiol Funct Imaging. 2016;36:499-503.

68. Ishiwata Y, Kaneta T, Nawata S, et al. Quantification of temporal changes in calcium score in active atherosclerotic plaque in major vessels by (18)F-sodium fluoride PET/CT. Eur J Nucl Med Mol Imaging. 2017;44:1529-37.

69. Irkle A, Vesey AT, Lewis DY, et al. Identifying active vascular microcalcification by (18)F-sodium fluoride positron emission tomography. Nat Commun. 2015;6:7495.

70. Alam MM, Lee J, Lee SY. Recent progress in the development of TSPO PET ligands for neuroinflammation imaging in neurological diseases. Nucl Med Mol Imaging. 2017;51(4):283-96.

71. Truillet $\mathrm{C}$, et al. Longitudinal imaging of microglia-astrocyte activation in mouse mesial temporal lobe epilepsy with TSPO PET to identify the best therapeutic time windows. Médecine Nucléaire. 2018;42(3):179-80.

72. Maeda $\mathrm{J}$, et al. In vivo positron emission tomographic imaging of glial responses to amyloid-beta and tau pathologies in mouse models of Alzheimer's disease and related disorders. J Neurosci. 2011;31(12):4720-30.

73. Dupont AC, et al. Translocator protein-18 kDa (TSPO) positron emission tomography (PET) imaging and its clinical impact in neurodegenerative diseases. Int J Mol Sci. 2017;18(4):E785.

74. Ji B, et al. Imaging of peripheral benzodiazepine receptor expression as biomarkers of detrimental versus beneficial glial responses in mouse models of Alzheimer's and other CNS pathologies. J Neurosci. 2008;28(47):12255-67.

75. Ji B, et al. Distinct binding of amyloid imaging ligands to unique amyloid-beta deposited in the presubiculum of Alzheimer's disease. J Neurochem. 2015;135(5):859-66.

76. Ishikawa A, et al. In vivo visualization of tau accumulation, microglial activation, and brain atrophy in a mouse model of tauopathy rTg4510. J Alzheimers Dis. 2018;61(3):1037-52.

77. Maeda $\mathbf{J}$, et al. Longitudinal, quantitative assessment of amyloid, neuroinflammation, and anti-amyloid treatment in a living mouse model of Alzheimer's disease enabled by positron emission tomography. J Neurosci. 2007;27(41):10957-68.

78. Ni R, et al. Comparative in vitro and in vivo quantifications of pathologic tau deposits and their association with neurodegeneration in tauopathy mouse models. J Nucl Med. 2018;59(6):960-6.

79. Gargiulo S, et al. Imaging of brain TSPO expression in a mouse model of amyotrophic lateral sclerosis with (18) F-DPA-714 and micro-PET/CT. Eur J Nucl Med Mol Imaging. 2016;43(7):1348-59.

80. Walker MD, et al. [11C]PBR28 PET imaging is sensitive to neuroinflammation in the aged rat. J Cereb Blood Flow Metab. 2015;35(8):1331-8.

81. Gerhard A. TSPO imaging in parkinsonian disorders. Clin Transl Imaging. 2016;4:183-90.

82. Varnas K, et al. PET imaging of $[(11) \mathrm{C}] \mathrm{PBR} 28$ in Parkinson's disease patients does not indicate increased binding to TSPO 
despite reduced dopamine transporter binding. Eur J Nucl Med Mol Imaging. 2019;46(2):367-75.

83. Morrice JR, Gregory-Evans CY, Shaw CA. Animal models of amyotrophic lateral sclerosis: a comparison of model validity. Neural Regen Res. 2018;13(12):2050-4.

84. Jankowsky JL, Zheng H. Practical considerations for choosing a mouse model of Alzheimer's disease. Mol Neurodegener. 2017;12(1):89.

85. Owen DR, et al. An 18-kDa translocator protein (TSPO) polymorphism explains differences in binding affinity of the PET radioligand PBR28. J Cereb Blood Flow Metab. 2012;32(1):1-5.

86. Rizzo G, et al. Kinetic modeling without accounting for the vascular component impairs the quantification of [(11)C]PBR28 brain PET data. J Cereb Blood Flow Metab. 2014;34(6):1060-9.

87. Tomasi G, et al. Novel reference region model reveals increased microglial and reduced vascular binding of 11C-(R)-PK11195 in patients with Alzheimer's disease. J Nucl Med. 2008;49(8):1249-56.

88. Horti AG, et al. PET imaging of microglia by targeting macrophage colony-stimulating factor 1 receptor (CSF1R). Proc Natl Acad Sci U S A. 2019;116(5):1686-91.

89. Tanzey SS, et al. Synthesis and initial in vivo evaluation of [(11) C]AZ683 - a novel PET radiotracer for colony stimulating factor 1 receptor (CSF1R). Pharmaceuticals (Basel). 2018;11(4):136.

90. Beaino W, et al. Purinergic receptors P2Y12R and P2X7R: potential targets for PET imaging of microglia phenotypes in multiple sclerosis. J Neuroinflammation. 2017;14(1):259.

91. Janssen B, et al. Identification of the allosteric P2X7 receptor antagonist [(11)C]SMW139 as a PET tracer of microglial activation. Sci Rep. 2018;8(1):6580.

92. Territo PR, et al. Characterization of (11)C-GSK1482160 for targeting the $\mathrm{P} 2 \mathrm{X} 7$ receptor as a biomarker for neuroinflammation. $\mathrm{J}$ Nucl Med. 2017;58(3):458-65.

93. Berdyyeva T, et al. PET imaging of the P2X7 ion channel with a novel tracer [(18)F]JNJ-64413739 in a rat model of neuroinflammation. Mol Imaging Biol. 2019;21:871-8.

94. Kolb H, et al. Preclinical evaluation and non-human primate receptor occupancy study of (18)F-JNJ-64413739, a novel PET radioligand for P2X7 receptors. J Nucl Med. 2019;60:1154-9.

95. Koole M, et al. (18)F-JNJ-64413739, a novel PET ligand for the P2X7 ion channel: radiation dosimetry, kinetic modeling, testretest variability and occupancy of the P2X7 antagonist JNJ54175446. J Nucl Med. 2019;60:683-90.

96. Villa A, et al. Identification of new molecular targets for PET imaging of the microglial anti-inflammatory activation state. Theranostics. 2018;8(19):5400-18.

97. DiSabato DJ, Quan N, Godbout JP. Neuroinflammation: the devil is in the details. J Neurochem. 2016;139(Suppl 2):136-53.

98. Kawabori M, Yenari MA. Inflammatory responses in brain ischemia. Curr Med Chem. 2015;22(10):1258-77.

99. Hosomi S, Watabe T, Mori Y, et al. Inflammatory projections after focal brain injury trigger neuronal network disruption: an (18) F-DPA714 PET study in mice. Neuroimage Clin. 2018;20:946-54.

100. Chen WW, Zhang X, Huang WJ. Role of neuroinflammation in neurodegenerative diseases (review). Mol Med Rep. 2016;13(4):3391-6.

101. Prata J, Santos SG, Almeida MI, et al. Bridging autism spectrum disorders and schizophrenia through inflammation and biomarkers-pre-clinical and clinical investigations. J Neuroinflammation. 2017;14(1):179.

102. Vivash L, O'Brien TJ. Imaging microglial activation with TSPO PET: lighting up neurologic diseases? J Nucl Med. 2016;57(2):165-8.

103. Kreisl WC, Fujita M, Fujimura Y, et al. Comparison of $\left[{ }^{11} \mathrm{C}\right]-(\mathrm{R})-\mathrm{PK} 11195$ and $\left[{ }^{11} \mathrm{C}\right] \mathrm{PBR} 28$, two radioligands for translocator protein $(18 \mathrm{kDa})$ in human and monkey: implications for positron emission tomographic imaging of this inflammation biomarker. NeuroImage. 2010;49:2924-32.
104. Kobayashi M, Jiang T, Telu S, et al. ${ }^{11} \mathrm{C}-\mathrm{DPA}-713$ has much greater specific binding to translocator protein $18 \mathrm{kDa}$ (TSPO) in human brain than ${ }^{11} \mathrm{C}-(\mathrm{R})-\mathrm{PK} 11195$. J Cereb Blood Flow Metab. 2018;38:393-403.

105. Doorduin J, Klein HC, Dierckx RA, James M, Kassiou M, de Vries EF. $\left[{ }^{11} \mathrm{C}\right]$-DPA-713 and $\left[{ }^{18} \mathrm{~F}\right]-\mathrm{DPA}-714$ as new PET tracers for TSPO: a comparison with $\left[{ }^{11} \mathrm{C}\right]-(\mathrm{R})-\mathrm{PK} 11195$ in a rat model of herpes encephalitis. Mol Imaging Biol. 2009;11:386-98.

106. Owen DR, Yeo AJ, Gunn RN, et al. An 18-kDa translocator protein (TSPO) polymorphism explains differences in binding affinity of the PET radioligand PBR28. J Cereb Blood Flow Metab. 2012;32(1):1-5. https://doi.org/10.1038/jcbfm.2011.147.

107. Lavisse S, Guillermier M, Herard AS, et al. Reactive astrocytes overexpress TSPO and are detected by TSPO positron emission tomography imaging. J Neurosci. 2012;32:10809-18.

108. Watabe T, Kanai Y, Ikeda H, et al. Evaluation of recanalization of occluded middle cerebral artery and restored cerebral blood flow in rats with transient brain ischemia: a combination study of digital subtraction angiography and ${ }^{15} \mathrm{O}$-water positron emission tomography. Cereb Blood Flow Metabolism. 2015;26(2):1-9.

109. Koizumi J, Yoshida Y, Nakazawa T, Oneda G. Experimental studies of ischemic brain edema. A new experimental model of cerebral embolism in rats in which recirculation can be introduced in the ischemia area. Jpn J Stroke. 1986;8:1-8.

110. Martin A, Boisgard R, Theze B, et al. Evaluation of the PBR/ TSPO radioligand [(18)F]DPA-714 in a rat model of focal cerebral ischemia. J Cereb Blood Flow Metab. 2010;30(1):230-41.

111. Martin A, Boisgard R, Kassiou M, Dolle F, Tavitian B. Reduced $\mathrm{PBR} / \mathrm{TSPO}$ expression after minocycline treatment in a rat model of focal cerebral ischemia: a PET study using [(18)F]DPA-714. Mol Imaging Biol. 2011;13(1):10-5.

112. Jin X, Ishii H, Bai Z, Itokazu T, Yamashita T. Temporal changes in cell marker expression and cellular infiltration in a controlled cortical impact model in adult male C57BL/6 mice. PLoS One. 2012;7(7): e41892.

113. Hosomi S, Koyama Y, Watabe T, Ohnishi M, Ogura H, Yamashita $\mathrm{T}$, et al. Myeloid-derived suppressor cells infiltrate the brain and suppress neuroinflammation in a mouse model of focal traumatic brain injury. Neuroscience. 2019;406:457-66. https://doi. org/10.1016/j.neuroscience.2019.03.015.

114. Maeda J, Zhang MR, Okauchi T, Ji B, Ono M, Hattori S, et al. In vivo positron emission tomographic imaging of glial responses to amyloid-beta and tau pathologies in mouse models of Alzheimer's disease and related disorders. J Neurosci. 2011;31(12):4720-30.

115. Ji B, Maeda J, Sawada M, Ono M, Okauchi T, Inaji M, et al. Imaging of peripheral benzodiazepine receptor expression as biomarkers of detrimental versus beneficial glial responses in mouse models of Alzheimer's and other CNS pathologies. J Neurosci. 2008;28(47):12255-67.

116. Maier FC, Wehrl HF, Schmid AM, et al. Longitudinal PET-MRI reveals beta-amyloid deposition and rCBF dynamics and connects vascular amyloidosis to quantitative loss of perfusion. Nat Med. 2014;20(12):1485-92.

117. Shukuri M, Takashima-Hirano M, Tokuda K, Takashima T, Matsumura $\mathrm{K}$, Inoue $\mathrm{O}$, et al. In vivo expression of cyclooxygenase- 1 in activated microglia and macrophages during neuroinflammation visualized by PET with $11 \mathrm{C}$-ketoprofen methyl ester. J Nucl Med. 2011;52(7):1094-101.

118. Shukuri M, Mawatari A, Ohno M, Suzuki M, Doi H, Watanabe Y, et al. Detection of cyclooxygenase-1 in activated microglia during amyloid plaque progression: PET studies in Alzheimer's disease model mice. J Nucl Med. 2016;57(2):291-6.

119. Imamoto N, Momosaki S, Fujita M, Omachi S, Yamato H, Kimura $\mathrm{M}$, et al. [11C]PK11195 PET imaging of spinal glial activation after nerve injury in rats. NeuroImage. 2013;79:121-8.

120. Belloli S, Zanotti L, Murtaj V, Mazzon C, Di Grigoli G, Monterisi $\mathrm{C}$, et al. (18)F-VC701-PET and MRI in the in vivo neuroinflam- 
mation assessment of a mouse model of multiple sclerosis. $\mathrm{J}$ Neuroinflammation. 2018;15(1):33.

121. Higuchi M, Ji B, Maeda J, et al. In vivo imaging of neuroinflammation in Alzheimer's disease. Clin Experiment Neuroimmunol. 2016;7:139-44.

122. Parvathenani LK, Tertyshnikova S, Greco CR, Roberts SB, Robertson B, Posmantur R. P2X7 mediates superoxide production in primary microglia and is up-regulated in a transgenic mouse model of Alzheimer's disease. J Biol Chem. 2003;278(15):13309-17.

123. Moore CS, Ase AR, Kinsara A, Rao VT, Michell-Robinson M, Leong SY, et al. P2Y12 expression and function in alternatively activated human microglia. Neurol Neuroimmunol Neuroinflamm. 2015;2(2):e80.

124. Kolb H, Barret O, Bhattacharya A, Chen G, Constantinescu C, Huang C, et al. Preclinical evaluation and non-human primate receptor occupancy study of (18)F-JNJ-64413739, a novel PET radioligand for P2X7 receptors. J Nucl Med. 2019;60:1154-9.

125. Koole M, Schmidt M, Hijzen A, Ravenstijn P, Vandermeulen C, Van Weehaeghe D, et al. (18)F-JNJ-64413739, a novel PET ligand for the P2X7 ion channel: radiation dosimetry, kinetic modeling, test-retest variability and occupancy of the P2X7 antagonist JNJ54175446. J Nucl Med. 2019;60:683-90.

126. Fu Z, Lin Q, Hu B, Zhang Y, Chen W, Zhu J, et al. P2X7 radioligand (18)F-PTTP for the differentiation of lung tumor and inflammation. J Nucl Med. 2019; https://doi.org/10.2967/ jnumed.118.222547.

127. Narayanaswami V, Dahl K, Bernard-Gauthier V, Josephson L, Cumming P, Vasdev N. Emerging PET radiotracers and targets for imaging of neuroinflammation in neurodegenerative diseases: outlook beyond TSPO. Mol Imaging. 2018;17:1536012118792317. https://doi.org/10.1177/1536012118792317.

128. Papadopoulos V, Baraldi M, Guilarte TR, et al. Translocator protein $(18 \mathrm{kDa})$ : new nomenclature for the peripheral-type benzodiazepine receptor based on its structure and molecular function. Trends Pharmacol Sci. 2006;27:402-9.

129. Zhang MR, Kida T, Noguchi J, Furutsuka K, Maeda J, Suhara T, Suzuki K. $\left[{ }^{11} \mathrm{C}\right]$ DAA1106: radiosynthesis and in vivo binding to peripheral benzodiazepine receptors in mouse brain. Nucl Med Biol. 2003;30:513-9.

130. Maeda J, Suhara T, Zhang MR, et al. Novel peripheral benzodiazepine receptor ligand $\left[{ }^{11} \mathrm{C}\right] \mathrm{DAA} 1106$ for PET: an imaging tool for glial cells in the brain. Synapse. 2004;52:283-91.

131. Ikoma Y, Yasuno F, Ito H, et al. Quantitative analysis for estimating binding potential of the peripheral benzodiazepine receptor with $\left[{ }^{11}\right.$ C]DAA1106. J Cereb Blood Flow Metab. 2007;27:173-84.

132. Zhang MR, Maeda J, Furutsuka K, et al. $\left[{ }^{18}\right.$ F]FMDAA1106 and $\left[{ }^{18}\right.$ F]FEDAA1106: two positron-emitter labeled ligands for peripheral benzodiazepine receptor (PBR). Bioorg Med Chem Lett. 2003;13:201-4.

133. Zhang MR, Maeda J, Ogawa M, et al. Development of a new radioligand, $\mathrm{N}$-(5-fluoro-2-phenoxyphenyl)- $\mathrm{N}-\left(2-\left[{ }^{18} \mathrm{~F}\right]\right.$ fluoroethyl-5-methoxybenzyl)acetamide, for pet imaging of peripheral benzodiazepine receptor in primate brain. J Med Chem. 2004;47:2228-35

134. Fujimura Y, Ikoma Y, Yasuno F, et al. Quantitative analyses of ${ }^{18}$ F-FEDAA1106 binding to peripheral benzodiazepine receptors in living human brain. J Nucl Med. 2006;47:43-50

135. Zhang MR, Kumata K, Maeda J, et al. ${ }^{11} \mathrm{C}-\mathrm{AC}-5216$ : a novel PET ligand for peripheral benzodiazepine receptors in the primate brain. J Nucl Med. 2007;48:1853-61.

136. Miyoshi M, Ito H, Arakawa R, et al. Quantitative analysis of peripheral benzodiazepine receptor in the human brain using PET with ${ }^{11} \mathrm{C}-\mathrm{AC}-5216$. J Nucl Med. 2009;50:1095-101.

137. Yanamoto K, Kumata K, Yamasaki T, et al. $\left[{ }^{18} \mathrm{~F}\right]$ FEAC and $\left[{ }^{18} \mathrm{~F}\right]$ FEDAC: two novel positron emission tomography ligands for peripheral-type benzodiazepine receptor in the brain. Bioorg Med Chem Lett. 2009;19:1707-10.

138. Yui J, Maeda J, Kumata K, et al. ${ }^{18}$ F-FEAC and ${ }^{18}$ F-FEDAC: PET of the monkey brain and imaging of translocator protein $(18 \mathrm{kDa})$ in the infarcted rat brain. J Nucl Med. 2010;51:1301-9.

139. Kawamura K, Kumata K, Takei M, et al. Efficient radiosynthesis and non-clinical safety tests of the TSPO radioprobe $\left[{ }^{18} \mathrm{~F}\right]$ FEDAC: prerequisites for clinical application. Nucl Med Biol. 2016;43:445-53.

140. Yanamoto K, Kumata K, Fujinaga M, et al. In vivo imaging and quantitative analysis of TSPO in rat peripheral tissues using smallanimal PET with [ ${ }^{18}$ F]FEDAC. Nucl Med Biol. 2010;37:853-60.

141. Hatori A, Yui J, Yamasaki T, et al. PET imaging of lung inflammation with $\left[{ }^{18} \mathrm{~F}\right] \mathrm{FEDAC}$, a radioligand for translocator protein $(18$ kDa). PLoS One. 2012;7:e45065.

142. Xie L, Yui J, Hatori A, et al. Translocator protein ( $18 \mathrm{kDa})$, a potential molecular imaging biomarker for non-invasively distinguishing non-alcoholic fatty liver disease. J Hepatol. 2012;57:1076-82.

143. Hatori A, Yui J, Xie L, et al. Visualization of acute liver damage induced by cycloheximide in rats using PET with $\left[{ }^{18} \mathrm{~F}\right] \mathrm{FEDAC}$, a radiotracer for translocator protein $(18 \mathrm{kDa})$. PLoS One. 2014;9:e86625.

144. Hatori A, Yui J, Xie L, et al. Utility of translocator protein (18 kDa) as a molecular imaging biomarker to monitor the progression of liver fibrosis. Sci Rep. 2015;5:17327.

145. Xie L, Yamasaki T, Ichimaru N, et al. $\left[{ }^{11} \mathrm{C}\right] \mathrm{DAC}-\mathrm{PET}$ for noninvasively monitoring neuroinflammation and immunosuppressive therapy efficacy in rat experimental autoimmune encephalomyelitis model. J Neuroimmune Pharmacol. 2012;7:231-42.

146. Chung SJ, Yoon HJ, Youn $\mathrm{H}$, et al. ${ }^{18} \mathrm{~F}-\mathrm{FEDAC}$ as a targeting agent for activated macrophages in DBA/1 mice with collagen-induced arthritis: comparison with ${ }^{18} \mathrm{~F}-\mathrm{FDG}$. J Nucl Med. 2018;59:839-45.

147. Chung SJ, Youn H, Jeong EJ, et al. In vivo imaging of activated macrophages by ${ }^{18} \mathrm{~F}-\mathrm{FEDAC}$, a TSPO targeting PET ligand, in the use of biologic disease-modifying anti-rheumatic drugs (bDMARDs). Biochem Biophys Res Commun. 2018;506:216-22.

148. Cunningham C. Microglia and neurodegeneration: the role of systemic inflammation. Glia. 2013;61:71-90.

149. Rezaie P, Patel K, Male DK. Microglia in the human fetal spinal cord; patterns of distribution, morphology and phenotype. Dev Brain Res. 1999;115:71-8.

150. Ren L, Lubrich B, Biber K, Gebicke-Haerter PJ. Mol Brain Res. 1999;65:198-205.

151. Suzumura A, Sawada M, Takayanagi T. Production of interleukin12 and expression of its receptors by murine microglia. Brain Res. 1998;787:139-42.

152. Katoh Y, Niimi M, Yamamoto Y, Kawamura T, MorimotoIshizuka T, Sawada M, Takemori H, Yamatodani A. Histamine production by cultured microglial cells of the mouse. Neurosci Lett. 2001;305:181-4.

153. Okada M, Irie S, Sawada M, Urae R, Urae A, Iwata N, Ozaki N, Akazawa K, Nakanishi H. Pepstatin a induces extracellular acidification distinct from aspartic protease inhibition in microglial cell lines. Glia. 2003:43:167-74.

154. Shimizu E, Kawahara K, Kajizono M, Sawada M, Nakayama H. Interleukin-4-induced selective clearance of oligomeric betaamyloid peptide $1-42$ by rat primary type- 2 microglia. J Immunol. 2008;181:6503-13.

155. Ling EA, Wong WC. The origin and nature of ramified and amoeboid microglia: a historical review and current concepts. Glia. 1993;7:9-18.

156. Sawada M, Suzumura A, Yamamoto H, Marunouchi T. Activation and proliferation of the isolated microglia by colony stimulating factor- 1 and possible involvement of protein kinase C. Brain Res. 1990;509:119-24.

157. Witmer-Pack MD, Hughes DA, Schuler G, Lawson L, McWilliam A, Inaba K, Steinman RM, Gordon S. Identification of macro- 
phages and dendritic cells in the osteopetrotic (op/op) mouse. J Cell Sci. 1993;104:1021-9.

158. Wiktor JW, Bartocci A, Ferrante AJ, Ahmed AA, Sell KW, Pollard JW, Stanley ER. Total absence of colony-stimulating factor 1 in the macrophage-deficient osteopetrotic (op/op) mouse. Proc Natl Acad Sci U S A. 1990;87:4828-32.

159. Yoshida H, Hayashi S, Kunisada T, Ogawa M, Nishikawa S, Okamura H, Sudo T, Shultz LD, Nishikawa S. The murine mutation osteopetrosis is in the coding region of the macrophage colony stimulating factor gene. Nature. 1990;345:442-4.

160. Gehrmann J, Matsumoto Y, Kreutzberg GW. Microglia: intrinsic immuneffector cell of the brain. Brain Res Brain Res Rev. 1995;20:269-87.

161. Ziegler-Heitbrock HWL, Ulevitch RJ. CD14: cell surface receptor and differentiation marker. Immunol Today. 1993;14:121-5.

162. Freeman MW. Macrophage scavenger receptors. Curr Opin Lipidol. 1994;5:143-8.

163. Suzumura A, Marunouchi T, Yamamoto H. Morphological transformation of microglia in vitro. Brain Res. 1991;545:301-6.

164. Sawada M, Suzumura A, Itoh Y, Marunouchi T. Production of interleukin- 5 by mouse astrocytes and microglia in culture. Neurosci Lett. 1993;155:175-8.

165. Sawada M, Suzumura A, Marunouchi T. Induction of functional interleukin-2 receptor in mouse microglia. J Neurochem. 1995;64:1973-9.

166. Kovacs E, Brock B, Varesio L, Young H. IL-2 induction of IL-1 beta mRNA expression in monocytes. J Immunol. 1989;143:3532-7.

167. Wahl S, McCartney-Francis N, Hunt D, Smith P, Wahl L, Katona I. Monocyte interleukin-2-receptor gene expression and interleukin-2 augmentation of microbicidal activity. J Immunol. 1987;139:1342-7.

168. Sawada M, Suzumura A, Marunouchi T. Down regulation of CD4 expression in cultured microglia by immunosuppressants and lipopolysaccharide. Biochem Biophys Res Commun. 1992;189:869-76.

169. Lassmann H, Schmied M, Vass K, Hickey WF. Bone marrow derived elements and resident microglia in brain inflammation. Glia. 1993;7:19-24.

170. Matsumoto Y, Fujiwara M. Absence of donor-type major histocompatibility complex class I antigen- bearing microglia in the rat central nervous system of radiation bone marrow chimeras. $\mathrm{J}$ Neuroimmunol. 1987;17:71-82.

171. MatsumotoY,HaraN,TanakaR,FujiwaraM.Immunohistochemical analysis of the rat central nervous system during experimental allergic encephalomyelitis, with special reference to Ia-positive cells with dendritic morphology. J Immunol. 1986;136:3668-76.

172. Rocha B, von BH, Guy GD. Selection of intraepithelial lymphocytes with CD8 alpha/alpha co-receptors by self-antigen in the murine gut. Proc Natl Acad Sci U S A. 1992;89:5336-40.

173. Nakata K, Akagawa KS, Fukayama M, Hayashi Y, Kadokura M, Tokunaga T. Granulocyte-macrophage colony-stimulating factor promotes the proliferation of human alveolar macrophages in vitro. J Immunol. 1991;147:1266-72.

174. Dexter DT, Nanayakkara I, Goss-Sampson MA, Muller DP, Harding AE, Marsden CD, et al. Nigral dopaminergic cell loss in vitamin E deficient rats. Neuroreport. 1994;5:1773-6.

175. Nagatsu T, Sawada M. Cellular and molecular mechanisms of Parkinson's disease: neurotoxins, causative genes, and inflammatory cytokines. Cell Mol Neurobiol. 2006;26:781-802.
176. Sawada M, Imamura K, Nagatsu T. Role of cytokines in inflammatory process in Parkinson's disease. J Neural Transm Suppl. 2006;70:373-81.

177. Imamura K, Hishikawa N, Sawada M, Nagatsu T, Yoshida M, Hashizume Y. Distribution of major histocompatibility complex class Il-positive microglia and cytokine profile of Parkinson's disease brains. Acta Neuropathol. 2003;106:518-26.

178. Wu DC, Jackson-Lewis V, Vila M, Tieu K, Teismann P, Vadseth $\mathrm{C}$, et al. Blockade of microglial activation is neuroprotective in the 1methyl-4-phenyl-1,2,3,6-tetrahydropyridine mouse model of Parkinson disease. J Neurosci. 2002;22:1763-71.

179. Wu DC, Teismann P, Tieu K, Vila M, Jackson-Lewis V, Ischiropoulos $\mathrm{H}$, et al. NADPH oxidase mediates oxidative stress in the 1-methyl4-phenyl-1,2,3,6-tetrahydropyridine model of Parkinson disease. Proc Natl Acad Sci U S A. 2003;100:6145-50.

180. Ito S, Sawada M, Haneda M, Ishida Y, Isobe K. Amyloid-beta peptides induce several chemokine mRNA expressions in the primary microglia and Ra2 cell line via PI3K/Akt and/or ERK pathway. Neurosci Res. 2006;56:294-9.

181. Sawada H, Hashida R, Hirata Y, Ono K, Suzuki H, Muramatsu SI, et al. Activated microglia affect the nigro-striatal dopamine neurons differently in neonatal and aged mice treated with 1-methyl-4-phenyl 1,2,3,6-tetra-hydropyridine. J Neurosci Res. 2007;85:1752-61.

182. Imai F, Suzuki H, Oda J, Ninomiya T, Ono K, Sano H, et al. Neuroprotective effect of exogenous microglia in global brain ischemia. J Cereb Blood Flow Metab. 2007;27:488-500.

183. Vilhardt F, Plastre O, Sawada M, Suzuki K, Wiznerowicz M, Kiyokawa E, et al. The HIV-1 Nef protein and phagocyte NADPH oxidase activation. J Biol Chem. 2002;277:42136-43.

184. Sawada M, Suzumura A, Marunouchi T. Cytokine network in the central nervous system and its roles in growth and differentiation of glial and neuronal cells. Int J Dev Neurosci. 1995;13:253-64.

185. Alderman TS, Frothingham R, Sempowski GD. Validation of an animal isolation imaging chamber for use in animal biosafety level-3 containment. Appl Biosaf. 2010;15:62-6.

186. Lei XY, Liu MM, Yu XJ. Severe fever with thrombocytopenia syndrome and its pathogen SFTSV. Microbes Infect. 2015;17:149-54.

187. Hayasaka D, Nishi K, Fuchigami T, et al. 18F-FDG PET imaging for identifying the dynamics of intestinal disease caused by SFTSV infection in a mouse model. Oncotarget. 2016;7:140-7.

188. Fuchigami T, Ono H, Oyadomari K, et al. Development of a (68) $\mathrm{Ge} /(68) \mathrm{Ga}$ generator system using polysaccharide polymers and its application in PET imaging of tropical infectious diseases. ACS. Omega. 2017;2:1400-7.

189. Sauerbrunn BJ, Andrews GA, Hubner KF. Ga-67 citrate imaging in tumors of the genito-urinary tract: report of cooperative study. $\mathrm{J}$ Nucl Med. 1978;19:470-5.

190. Rossleigh MA, Murray IP, Mackey DW, Bargwanna KA, Nayanar VV. Pediatric solid tumors: evaluation by gallium-67 SPECT studies. J Nucl Med. 1990;31:168-72.

191. Tsan MF. Mechanism of gallium-67 accumulation in inflammatory lesions. J Nucl Med. 1985;26:88-92.

192. Mougneau E, Bihl F, Glaichenhaus N. Cell biology and immunology of Leishmania. Immunol Rev. 2011;240:286-96. 